University of Tennessee Health Science Center

UTHSC Digital Commons

\title{
Antibodies to Heterogenous Nuclear Ribonucleoprotein A1 Penetrate Neurons Leading to Multiple Downstream Effects Resulting in Neurodegeneration
}

Joshua Nathan Douglas

University of Tennessee Health Science Center

Follow this and additional works at: https://dc.uthsc.edu/dissertations

Part of the Amino Acids, Peptides, and Proteins Commons, Immune System Diseases Commons, Nervous System Diseases Commons, and the Neurosciences Commons

\section{Recommended Citation}

Douglas, Joshua Nathan (http://orcid.org/0000-0001-5140-0133), "Antibodies to Heterogenous Nuclear Ribonucleoprotein A1 Penetrate Neurons Leading to Multiple Downstream Effects Resulting in Neurodegeneration" (2016). Theses and Dissertations (ETD). Paper 391. http://dx.doi.org/10.21007/ etd.cghs.2016.0402.

This Dissertation is brought to you for free and open access by the College of Graduate Health Sciences at UTHSC Digital Commons. It has been accepted for inclusion in Theses and Dissertations (ETD) by an authorized administrator of UTHSC Digital Commons. For more information, please contact jwelch30@uthsc.edu. 


\title{
Antibodies to Heterogenous Nuclear Ribonucleoprotein A1 Penetrate Neurons Leading to Multiple Downstream Effects Resulting in Neurodegeneration
}

\author{
Abstract \\ Multiple sclerosis (MS) is the most common demyelinating disorder of the central nervous system. MS is \\ believed to occur in genetically susceptible individuals due to an unknown environmental stimulus. MS \\ patients produce autoantibodies to heterogenous nuclear ribonuclearprotein A1 (hnRNP A1), an RNA \\ binding protein (RBP) highly expressed in neurons. hnRNP A1 functions in pre-mRNA splicing, mRNA \\ trafficking, and translation. Furthermore, the anti-hnRNP A1 antibodies are specific to a $\mathrm{N}$-terminal region \\ termed ' $M 9$ ' which serves as a nuclear export sequence/nuclear localization sequence (NES/NLS) \\ responsible for nuclear/cytoplasmic transport of the protein. In this manuscript we will provide data \\ revealing that anti-hnRNP A1 antibodies enter neuronal cells via clathrin-mediated endocytosis. Moreover, \\ we have shown that anti-hnRNP A1 antibodies cause redistribution of endogenous hnRNP A1 protein, \\ decrease in cellular ATP levels, and increase in apoptosis. Additionally, we present data depicting RNA \\ binding partners of hnRNP A1 protein as well as the effect of anti-hnRNP A1 autoantibodies upon specific \\ RNA binding partners. To further these studies we set out to determine the effect of anti-hnRNP A1 \\ antibodies on experimental autoimmune encephalomyelitis (EAE), the murine model of MS. Specifically, \\ we will present the effect of anti-hnRNP A1-M9 antibodies on clinical symptoms and neurodegeneration \\ in EAE. Taken together, we present a series of experiments which will depict a journey of discovery from \\ the initial discovery of anti-hnRNP A1 antibodies to our present understanding of trafficking, deleterious \\ effects, and in vivo effects of anti-hnRNP A1 antibodies and their implications in the disease Multiple \\ Sclerosis.

\section{Document Type} \\ Dissertation \\ Degree Name \\ Doctor of Philosophy (PhD) \\ Program \\ Biomedical Sciences \\ Research Advisor \\ Michael C. Levin, M.D. \\ Keywords \\ EAE, hnRNP A1, immunology, Multiple sclerosis, neurodegeneration, RNA binding protein

\section{Subject Categories} \\ Amino Acids, Peptides, and Proteins | Chemicals and Drugs | Diseases | Immune System Diseases | \\ Medical Sciences | Medicine and Health Sciences | Nervous System Diseases | Neurosciences
}


Antibodies to Heterogeneous Nuclear Ribonucleoprotein A1 Penetrate Neurons Leading to Multiple Downstream Effects Resulting in Neurodegeneration

\author{
A Dissertation \\ Presented for \\ The Graduate Studies Council \\ The University of Tennessee \\ Health Science Center
}

\begin{abstract}
In Partial Fulfillment
Of the Requirements for the Degree

Doctor of Philosophy

From The University of Tennessee
\end{abstract}

By

Joshua Nathan Douglas

May 2016 
Copyright (C) 2016 by Joshua Nathan Douglas. All rights reserved. 


\section{DEDICATION}

This work is dedicated to all who have helped me along the way throughout my dissertation process, you know who you are. 


\section{ACKNOWLEDGEMENTS}

I would like to thank my mentor, Dr. Michael Levin, for his support and guidance throughout the entire process of my graduate education.

Thank you to Lidia Gardner for helping keep me sane and teaching me numerous techniques. Thank you for everything you did on a daily basis to help me throughout the process.

Thank you to my wife Allison for being supportive and helping me make it through until the end.

Thank you Lexi for helping me study, especially in the early years, I couldn't have made it without you.

Thank you to my family for all the support throughout the years.

Thanks to Sam for all the encouraging words and keeping me motivated to stick it out early on in the dissertation process.

I would like to thank all my committee members for their wonderful words of encouragement as well as insight into my work throughout the years. You all really helped me shape this project into something special. 


\begin{abstract}
Multiple sclerosis (MS) is the most common demyelinating disorder of the central nervous system. MS is believed to occur in genetically susceptible individuals due to an unknown environmental stimulus. MS patients produce autoantibodies to heterogenous nuclear ribonuclearprotein A1 (hnRNP A1), an RNA binding protein (RBP) highly expressed in neurons. hnRNP A1 functions in pre-mRNA splicing, mRNA trafficking, and translation. Furthermore, the anti-hnRNP A1 antibodies are specific to a N-terminal region termed 'M9' which serves as a nuclear export sequence/nuclear localization sequence (NES/NLS) responsible for nuclear/cytoplasmic transport of the protein. In this manuscript we will provide data revealing that anti-hnRNP A1 antibodies enter neuronal cells via clathrin-mediated endocytosis. Moreover, we have shown that anti-hnRNP A1 antibodies cause redistribution of endogenous hnRNP A1 protein, decrease in cellular ATP levels, and increase in apoptosis. Additionally, we present data depicting RNA binding partners of hnRNP A1 protein as well as the effect of anti-hnRNP A1 autoantibodies upon specific RNA binding partners. To further these studies we set out to determine the effect of anti-hnRNP A1 antibodies on experimental autoimmune encephalomyelitis (EAE), the murine model of MS. Specifically, we will present the effect of anti-hnRNP A1-M9 antibodies on clinical symptoms and neurodegeneration in EAE. Taken together, we present a series of experiments which will depict a journey of discovery from the initial discovery of anti-hnRNP A1 antibodies to our present understanding of trafficking, deleterious effects, and in vivo effects of anti-hnRNP A1 antibodies and their implications in the disease Multiple Sclerosis.
\end{abstract}




\section{TABLE OF CONTENTS}

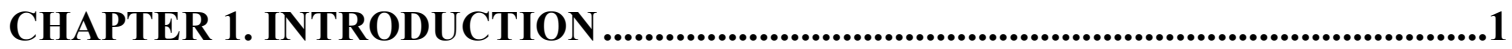

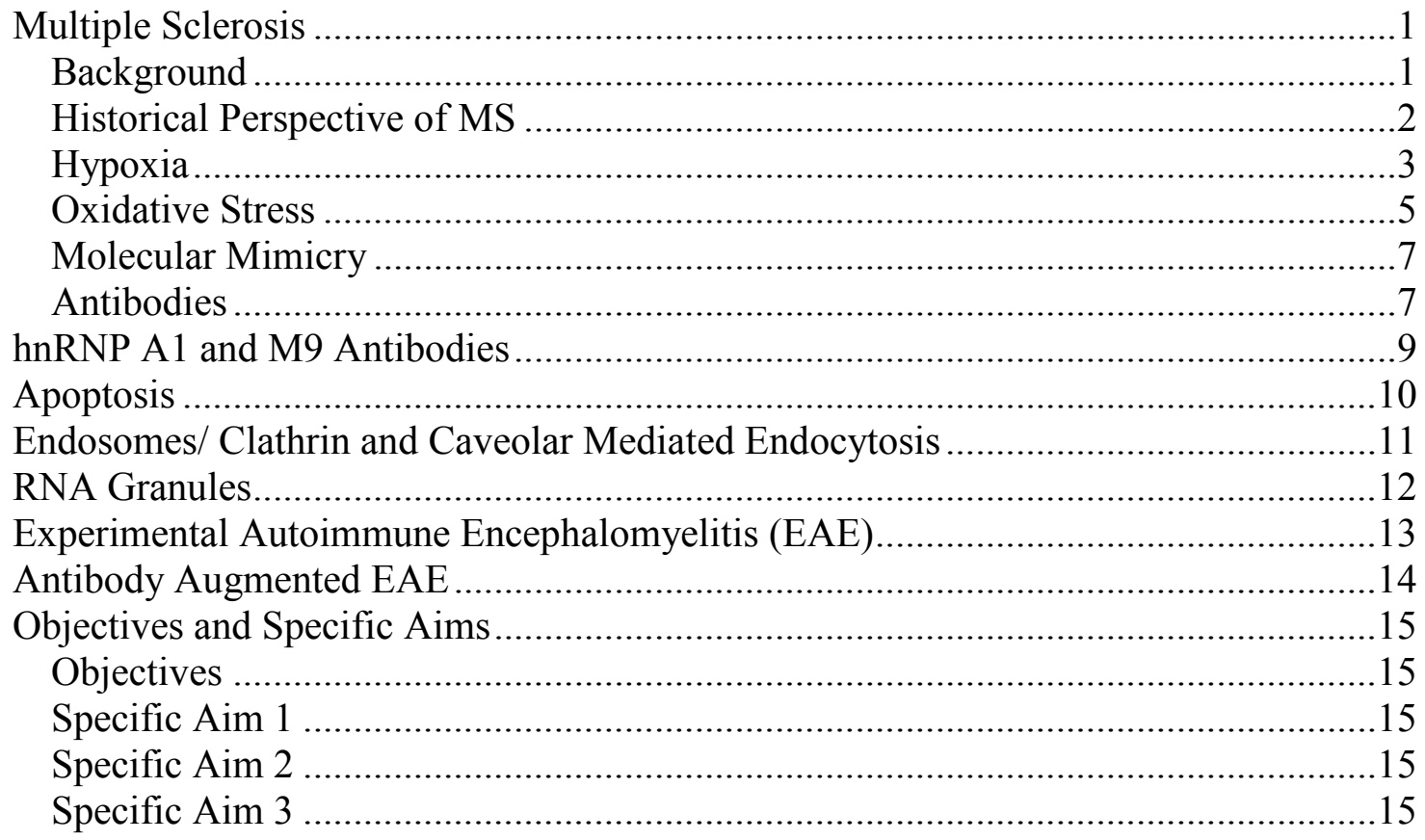

CHAPTER 2. MATERIALS AND METHODS.............................................................16

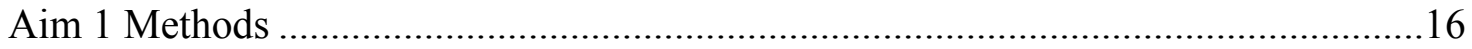

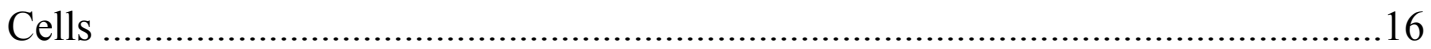

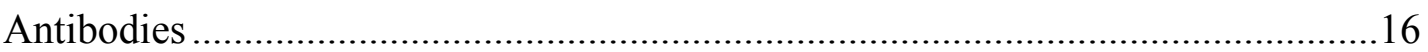

Atto550 NHS Labeling of Anti-hnRNP A1 and Normal IgG Antibodies ..................16

Antibody Penetration Tracing ..............................................................................

Anti-hnRNP A1 and Early Endosomes Co-localization............................................17

Determination of Clathrin or Caveolar Mediated Endocytosis....................................17

Measurement of ATP Levels ............................................................................. 18

Determination of Antibodies Effect on Caspase 3/7 Levels ......................................18

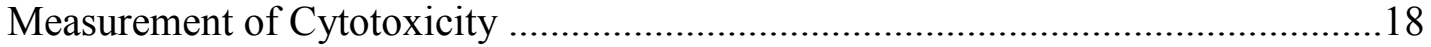

Double Labeling to Image Redistribution of Endogenous hnRNP A1 .....................19

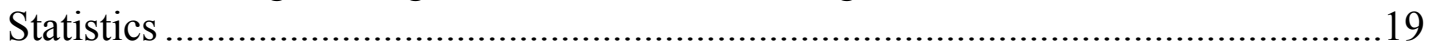

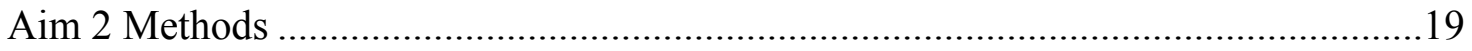

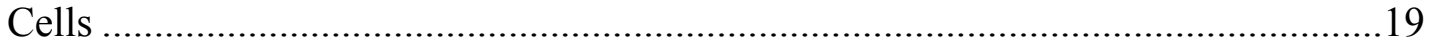

Exposure of SK-N-SH Cells to Antibodies in vitro ................................................19

RNA Immunoprecipitation .................................................................................2

Determination of Protein Levels of Antibody Treated SK-N-SH Cells by

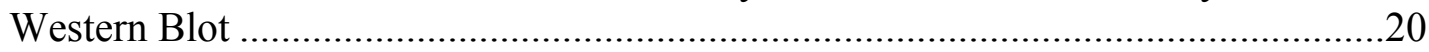

Immunocytochemistry of hnRNP A1-M9 Antibody Colocalization with

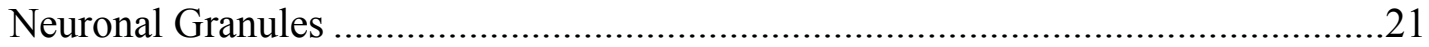

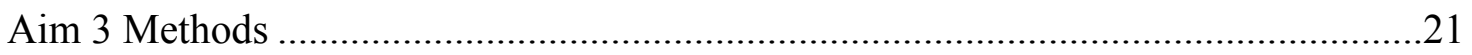

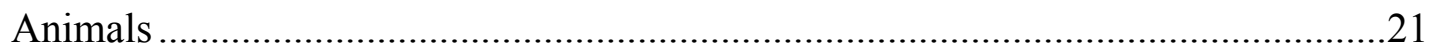


Induction of Experimental Autoimmune Encephalomyelitis/Antibody Injections....21

Clinical Scoring

Analyses of Neurodegeneration by Fluoro-Jade C Staining of Degenerating

Neural Elements

\section{CHAPTER 3. ANTI-HNRNP A1 ANTIBODIES PENETRATE AND CAUSE} DELETERIOUS EFFECTS IN NEURONS IN VITRO

Introduction

Direct Antibody Addition into Growth Media Results in Antibody Penetration into

Neuronal Cells

Antibodies to hnRNP A1 Penetrate Neuronal Cells and Co-localize with Early

Endosomes

Antibodies to hnRNP A1 and IgG Penetrate Neuronal Cells via Clathrin Mediated

Endocytosis and Not via Caveolar Mediated Endocytosis .

Anti-hnRNP A1 Antibodies Deplete Cellular ATP ................................................27

Anti-hnRNP A1 Antibodies Are Not Cytotoxic ...................................................29

Anti-hnRNP A1 Antibody Increased Apoptosis in Neuronal Cells ...........................29

Anti-hnRNP A1 Antibodies Cause Redistribution of Endogenous hnRNP A1

Protein

Conclusions

CHAPTER 4. ANTI-HNRNP A1 ANTIBODIES CO-LOCALIZE WITH RNA GRANULES AND ALTER SPASTIC PARAPLEGIA GENES IN NEURONS .......36

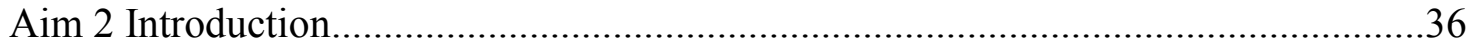

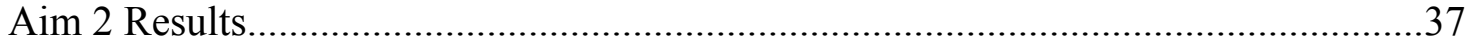

Anti-hnRNP A1 Antibodies Colocalize with SGs but Not P Bodies or Neuronal

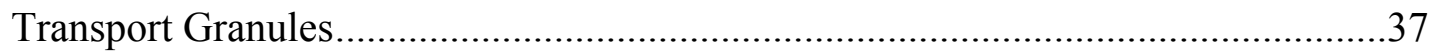

SPG4 and SPG7 RNA Bind hnRNP A1 Protein....................................................41

Anti-hnRNP A1-M9 Antibodies Alter SPG4 and SPG7 Protein Levels by

Western Blot

Aim 2 Conclusions

\section{CHAPTER 5. ANTI-HNRNP A1 ANTIBODIES INCREASE NEURODEGENERATION IN EXPERIMENTAL AUTOIMMUNE ENCEPHALOMYELITIS (EAE), AN ANIMAL MODEL OF MS}

Introduction

Results

Anti-hnRNP A1-M9 Antibodies Exacerbate $\mathrm{MOG}_{35-55}$ Induced EAE ......................48

Anti-hnRNP A1-M9 Antibodies Cause Increased Levels of Neurodegeneration

of Specific CNS Pathways in EAE

Conclusions for Aim 3

CHAPTER 6. DISCUSSION 


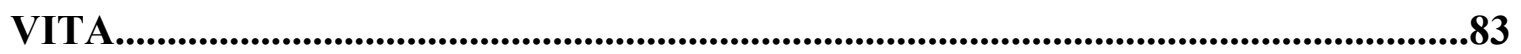

viii 


\section{LIST OF TABLES}

Table 4-1. Quantification of antibody colocalization with stress granules ..................40

Table 5-1. Areas of neurodegeneration in an antibody augmented model of EAE........53 


\section{LIST OF FIGURES}

Figure 3-1. Localization of antibodies by immunocytochemistry in SK-N-SH cells......25

Figure 3-2. Antibodies co-localize with early endosomes.

Figure 3-3. Antibodies (labeled red) penetrate SK-N-SH cells via clathrin-mediated endocytosis.

Figure 3-4. Anti-hnRNP A1 antibodies caused an increase in apoptosis and depletion of ATP levels.

Figure 3-5. Anti-hnRNP A1 antibodies cause redistribution of endogenous hnRNP A1 in SK-N-SH cells.

Figure 4-1. Antibodies co-localize with stress granules in SK-N-SH neurons.

Figure 4-2. RNA immunoprecipitation

Figure 4-3. Anti-hnRNP A1 antibodies alter protein levels as measured by Western blot.

Figure 5-1. Clinical scores of EAE mice.

Figure 5-2. Fluoro-jade C staining of the spinal cord.

Figure 5-3. Fluoro-jade C staining of the ventral spinocerebellar tract of the brain stem.

Figure 5-4. Fluoro-jade $\mathrm{C}$ staining of the deep cerebellar white matter.

Figure 5-5. Fluoro jade $\mathrm{C}$ staining of the corpus callosum. .56

Figure 5-6. Neural pathways related to the ventral spinocerebellar tract (VSCT). .58 


\section{CHAPTER 1. INTRODUCTION}

This dissertation is a compilation of work I completed in the lab of Dr. Michael Levin to fulfill the requirements for a Ph.D. in neuroscience. The background section entails a literature review on the role of neurodegeneration in the pathogenesis of multiple sclerosis (MS). It includes information on a number of hypotheses about neurodegeneration including, but not limited to, work related to my experiments on how autoantibodies to the RNA binding protein hnRNP A1 might contribute to neurodegeneration in MS. The work has been divided into the following three aims:

- Aim 1, "Anti-hnRNP A1 antibodies penetrate and cause deleterious effects in neurons in vitro," was published in the Journal of Clinical and Cellular Immunology ${ }^{1}$.

- Aim 2, "Anti-hnRNP A1 antibodies co-localize with RNA granules and alter spastic paraplegia genes in neurons," is a continuation of work describing mechanisms by which anti-hnRNP A1 antibodies cause neurodegeneration of neurons in vitro. A manuscript based on these experiments is under review by Neuroscience Letters.

- Aim 3, "Anti-hnRNP A1 antibodies increase neurodegeneration in experimental autoimmune encephalomyelitis (EAE), an animal model of MS," examines how anti-hnRNP A1 antibodies might contribute to neurodegeneration in vivo.

\section{Multiple Sclerosis}

\section{Background}

Multiple sclerosis (MS) is a complex autoimmune disease that impairs the central nervous system (CNS). MS is the most common demyelinating disorder of the CNS. The neurological disability and clinical course of the disease is highly variable and unpredictable from one patient to another. The cause of MS is still unknown but it is thought to occur in genetically susceptible individuals who develop disease due to a nongenetic trigger such as altered metabolism, a virus, or other environmental factors ${ }^{2}$.

Patients with MS generally present to the physician with any of the following symptoms: numbness/weakness of limbs, loss/blurred vision, tingling/pain, tremor, slurred speech, fatigue, bowel problems, or dizziness. There are three main clinical types of MS. First, is the relapsing remitting type of MS. During the relapsing-remitting phase, patients experience bouts of neurological symptoms (relapses) which will eventually improve partially or completely for months or even years (remissions). Patients with relapsingremitting MS (RRMS) sometimes develop a second phase of disease called secondary progressive MS (SPMS) during which they progress to a 'smoldering' chronic disease 
which evolves at a constant rate without remissions. The third type of MS is primary progressive MS (PPMS). Patients with PPMS present to the physician with initial neurological symptoms and from that point onward have a disease course with constant 'smoldering' disease progression without any remissions. MS patients develop progressive, irreversible, neurological disability associated with neuronal and axonal damage, collectively known as neurodegeneration. Neurodegeneration in MS involves multiple mechanisms including autoimmunity to CNS targets, axonal hypoxia and oxidative stress, mitochondrial dysfunction, and disturbances in lipid metabolism and nuclear receptors. ${ }^{3-6}$ MS has historically been viewed primarily as a white matter disease. However, recent data indicates that both white matter and gray matter destruction contribute to its pathogenesis. While both inflammation and demyelination are well-described cellular processes, a diverse pool of any of the following might characterize neurodegeneration: neuronal cell death, apoptosis, necrosis, and hypoxia. Neurodegeneration was traditionally considered as a secondary phenomenon to inflammation and demyelination; however, some neurons undergo apoptosis at earlier stages of disease. Evidence suggests that an overlap between neurodegeneration, demyelination, and inflammation cause MS.

While demyelineation may plan an important role in relapsing remitting stage (RRMS), it doesn't correlate well with progressive forms of the disease. Evidence has While demyelineation may play an important role in relapsing remitting stage (RRMS), it doesn't correlate well with progressive forms of the disease. Evidence has suggested that the progressive stages of disease correlate more closely with axonal loss. ${ }^{7,8}$ Over the past several years a major shift in thinking about the pathogenesis of progressive forms of MS has occurred. ${ }^{8-20}$ Axonal loss rather than demyelination has been shown to correlate more accurately with clinical disability. ${ }^{18,21}$ A new concept emerging in the MS literature theorizes that axonal loss may be occurring independently of or even be the cause of the demyelination in MS. ${ }^{18,21}$ Evidence indicates that neurodegeneration occurs in all stages of the disease. ${ }^{7,9,13,16,22}$ In addition, neurodegeneration seen in progressive forms of MS does not correlate with white matter plaque location, but instead correlates with gray matter and cortical pathology. ${ }^{7,9,16,23-27}$ A post-mortem analysis of spinal cords from MS patients showed that axonal loss in white matter tracts did not associate with demyelinated plaques in the region. ${ }^{17}$ This indicates that there might be some pathological mechanisms independent of myelin loss that contributes to the axonal loss and neurodegeneration present in MS. Further evidence has shown that axonal injury can occur before myelin loss suggesting that axonal injury and neurodegeneration could be independent of demyelination and may occur prior to or in parallel with demyelination. $13,17,18,28$ Neurodegeneration is a very complicated process that involves several factors associated with the resulting clinical progression in MS patients.

\section{Historical Perspective of MS}

The first depiction of the disease which would be known as Multiple Sclerosis was illustrated by a Scotsman in $1838 .{ }^{29}$ The unnamed patient depicted was of French origin. Early on in the discovery of the disease various names and classifications existed. 
Jean-Martin Charcot (1825-1893), who many agree began the first serous studies on demyelinating disorders, referred to the disease as la sclerose en plaques disseminees, la sclerose multiloculaire, or la sclerose generalisee. ${ }^{29}$ These names were translated for the English-speaking world at the time as disseminated (cerebrospinal) sclerosis.

Throughout the early year's studies of the disease, various nomenclature was used throughout England, France, Australia and the new world. The term which we use today, multiple sclerosis originated in Germany. In Germany the term multiple Sklerose de Nervensystems was used from the onset. Consensus on nomenclatures was ultimately achieved in 1955 with the publication of Multiple Sclerosis, written by Douglas McAlpine, Nigel Compston, and Charles Lumsden. ${ }^{29}$

Throughout the history of MS, various scientific laboratory based findings have occurred which shed insight into varying aspects of the disease. In 1941, Elvin Kabat performed the first immunoglobulin $\mathrm{G}$ characterization, thus providing evidence of intrathecal antibody synthesis in MS patients. ${ }^{29}$ He further formed the framework for complementarity-determining regions of antibodies which led to the molecular basis of therapeutic humanization of monoclonal antibodies. In the modern era, John Whitaker is responsible for the revealing the role and specificity of oligoclonal banding in the CSF of patients with MS. In the 1960s new observations begin to implicate glia and remyelination in the pathogenesis of MS. ${ }^{29}$ By the late 1970s Ken Smith and his colleagues had provided proof that remyelination can restore conduction. The studies performed during this era which eventually led to the findings by Ken Smith and colleagues set the stage for an era of studies aiming to explore the neurobiology of remyelination as well as approaches to restore loss of this mechanism. ${ }^{29}$ More recently evidence has come to light which suggests axonal degeneration plays a vital role in the pathogenesis of $\mathrm{MS}^{8}$. As evidenced throughout the history of the disease, MS is a multifaceted disease in which novel findings have arisen periodically throughout history leading to the understanding we currently have today.

\section{Hypoxia}

Over the years, multiple hypotheses have been proposed to explain the pathogenesis of MS, ranging from viral infection, cytokine-induced apoptosis and oxidative stress to molecular mimicry and metabolic disorders. ${ }^{3,4,30,31}$ However, none successfully identified a single pathological mechanism, likely because MS is a heterogeneous disease with a multifaceted etiology. ${ }^{32,33}$ Although it has not been proven, the data presented could lead to the hypothesis that various disease causing entities likely lead to the form of MS present within each individual.

One school of thought suggests MS pathology is due to axonal damage and loss, which occurs when chronically demyelinated neurons reach a state of "virtual hypoxia" associated with reduced ATP production, ion channel and mitochondrial dysfunction. It is believed that loss of myelin results in an increased energy demand and a relative cellular energy deficit, which eventually leads to neuronal death. In a viable neuron, $\mathrm{Na}+/ \mathrm{K}+$ ATPase is located at Nodes of Ranvier. Nodes of Ranvier are gaps along the 
axon where the myelin sheath is not present. The Nodes of Ranvier conduct the electrical impulse along the axon. The Nodes of Ranvier contain $\mathrm{Na}+/ \mathrm{K}+\mathrm{ATPase}, \mathrm{Na}+/ \mathrm{Ca} 2+$ exchangers, and voltage gated $\mathrm{Na}+$ channels that generate action potentials along the axon. Evidence suggests that after demyelination, $\mathrm{Na}+$ channels undergo redistribution from a location predominantly on the Nodes of Ranvier to diffusely spread along the axon. ${ }^{34,35}$ Thus, the number of $\mathrm{NA}+/ \mathrm{K}+$ ATPase increases along a demyelinated axon in order to counteract the loss of $\mathrm{Na}+$ channels and continue saltatory conduction. The increase in $\mathrm{Na}+/ \mathrm{K}+$ ATPase results in an increased energy demand for neuronal firing. In MS patients this increased energy demand cannot be met because of the impaired mitochondrial energy production in the CNS. ${ }^{17,28,36}$ Impaired mitochondrial energy production leaves neurons in a depleted energy state, which has been shown to reduce the ability of $\mathrm{Na}^{+} / \mathrm{K}^{+}$ATPase function. ${ }^{37}$ Depleted mitochondrial energy production and reduced firing ability in the overpopulated $\mathrm{Na}^{+} / \mathrm{K}^{+}$ATPase within demyelinated neurons in MS leads to several deleterious downstream effects, among which is impaired neurotransmission. With a lack of efficient $\mathrm{Na}^{+} / \mathrm{K}^{+}$ATPase, the cell (in theory) should enter a state of axonal depolarization. This state of axonal depolarization causes the overpopulated $\mathrm{Na}^{+} / \mathrm{K}^{+}$ATPase to become leaky, resulting in increased intracellular $\mathrm{Na}+$ concentrations. It is believed that if axonal $\mathrm{Na}^{+}$rises to a concentration greater than 20 $\mathrm{mM}$, the $\mathrm{Na}^{+} / \mathrm{Ca}^{+}$exchanger will operate in reverse, thus acting as a system to dump $\mathrm{Ca}^{2+}$ into the axon. ${ }^{38}$ The increase in $\mathrm{Ca}^{2+}$ within the axon is known as $\mathrm{Ca}^{2+}$ loading. Additional sources may contribute to axonal $\mathrm{Ca}^{2+}$ loading such as release from intracellular $\mathrm{Ca}^{2+}$ stores, voltage gated $\mathrm{Ca}^{2+}$ channels, and $\mathrm{Ca}^{2+}$ permeable cation channels such as glutamate gated receptors. ${ }^{39-43}$ Large quantities of glutamate released by activated immune cells activate glutamate receptors, which result in axonal $\mathrm{Ca}^{2+}$ loading and subsequent neuronal death. ${ }^{44,45}$ In hypoxic cells reversal of $\mathrm{Na}^{+}$dependent glutamate transporters results in glutamate release. ${ }^{5}$ In addition, astrocytes can release glutamate by exocytosis or hemichannels and unmyelinated callosal axons release glutamate in a vesicular manner similar to normal release at the synapse. ${ }^{46-48}$ This increase in vesicular release of glutamate within the white matter has implications for the mechanisms of ischemia-induced myelin damage possibly through activation of glial cells. $^{46}$

Hypoxia might also play a role in formation of MS lesions. Decreased oxygen availability (hypoxia) is often seen in tissues at the sites of chronic inflammation. Inflamed tissue has increased metabolic activity due to the presence of inflammatory cells, poor perfusion related to blood vessel stenosis, and microthrombosis. Therefore, chronically inflamed tissue has increased demand and a limited supply of oxygen. This imbalance results in hypoxia at inflammatory sites. Hypoxia also increases the permeability of the blood brain barrier (BBB) and results in overexpression of proinflammatory genes. ${ }^{49-51}$ Patients with MS are seen to have increased permeability of the BBB. Hypoxia is often accompanied by hypoperfusion. In about $50 \%$ of MS patients, blood flow through normal-appearing white matter is reduced. ${ }^{52-56}$ Taken together, both hypoxia and hypoperfusion might be a precipitating factor for MS lesion formation.

Hypoxia-inducible factor (HIF) is an important transcription factor that regulates cellular metabolism and survival under hypoxic stress. HIF is composed of an alpha and 
beta subunit (HIF- $\alpha$ and HIF- $\beta$ ). Active HIF requires heterodimeric complex formation of the two subunits, which then translocate to the nucleus, binds to the hypoxia-response element (HRE), and associates with co-activators such as CBP/p300. The binding results in activation or suppression of many genes involved in metabolism and cell survival. These changes include an increase in hypoxia-inducible factor-1 $\alpha$ (HIF $1 \alpha$ ) protein levels, expression of HIF1 - inducible genes in MS brain, blood vessel density and endothelial

cell proliferation. ${ }^{49,57,58} \mathrm{HIF}-1 \alpha$ plays a pro-inflammatory role in cells of the myeloid lineage and anti-inflammatory role in intestinal epithelial cells. ${ }^{59,60}$ Increases in HIF-1 $\alpha$ could explain the increased inflammation seen in patients with MS, specifically in the RRMS stage when a predominantly $\mathrm{B}$ and $\mathrm{T}$ cell response is present.

HIFs regulate cellular stress responses in tandem with nuclear factor-kappa $\beta$ (NF-k-ß) to control hypoxic inflammation through activation of cytokine and hypoxia pathways. ${ }^{49}$ In fact there is a cross talk between these two transcription factors during hypoxic inflammation. HIF can be activated in response to multiple stimuli such as bacterial lipopolysaccharide, microtubule disruption, interleukin (IL-18) and tumor necrosis factor $\alpha$ (TNF- $\alpha$ ), hepatocyte growth factor and reactive oxygen species (ROS). The mechanism of HIF activation involves NF-k- $\beta$ dependent up-regulation of HIF-1 $\alpha$ messenger ribonucleic acid (mRNA) levels. ${ }^{61} \mathrm{HIF}-1 \alpha$ contains an active binding site for NF-k- $\beta$ upstream of the transcription start position. ${ }^{62}$ A recent study demonstrated that NF-k- $\beta$ controls the HIF pathway in response to TNF- $\alpha .{ }^{63}$ While NF-k- $\beta$ controls HIF- $1 \alpha$ expression levels, HIF-1 $\alpha$ can regulate NF-k- $\beta$ signaling. Mice overexpressing HIF- $1 \alpha$ exhibited increase in pro-inflammatory NF-k- $\beta$ targets. ${ }^{64}$ From data presented, it appears that HIFs could play an important role in the pathogenesis of MS. Although HIFs can act in a helpful manner in epithelial cells by acting in an anti-inflammatory manner, HIFs act as pro-inflammatory factors in the cells of myeloid lineage such as B and T cells. By acting in a pro-inflammatory manner, HIF alterations could be the source of the increased inflammatory cells seen in MS, especially in the RRMS stage when B and T cells predominate. Although this theory is not yet fully correlated, additional work within this area could spark important insight to the field of MS.

\section{Oxidative Stress}

Oxidative Stress (OS) resulting from the formation of reactive oxygen species (ROS) secreted primarily by macrophages is believed to play a role in the pathogenesis of $\mathrm{MS}^{2}$. ROS are free radicals and related molecules, which are defined as any chemical species that contain one or more unpaired electrons. ${ }^{3}$ The most common ROS are hydroxyl radical (OH-), superoxide radical (O2-), and nitric oxide (NO) as well as other molecules such as hydrogen peroxide (H2O2) and peroxynitrite (ONOO-). Unpaired electrons cause ROS to act as electron acceptors, which results in the stealing of electrons by ROS (oxidation). ROS occur within a normal cell to a certain extent and a number of mechanisms are in place to guard against ROS induced damage; however, it appears that in patients with MS the ROS exceed the capacity of the cellular defense mechanisms. ROS are known to cause damage to lipids, proteins, and DNA leading to cellular death by necrosis and apoptosis. Also, metals such as iron are normally stored within iron binding 
proteins. However injured cells release iron so it is available to catalyze the free-radical reactions of ROS formation. Other sources of free radical production are due to oxygen use in mitochondria and enzymatic pathways such as xanthine oxidase, NADPH oxidase, lipoxygenases, and cyclooxygenase. ${ }^{3}$ The respiratory burst system of activated microglia, which are glial cells that act as the immune response of the CNS, have also been shown to produce large quantities of ROS. In addition, reactive astrocytes have been shown to produce $\mathrm{NO}^{3,65}$

Evidence suggests that oxygen and nitrogen free radicals generated by macrophages cause demyelination and axonal injury in both EAE and MS ${ }^{66,67}$ EAE is an animal model of MS in which mice injected with myelin peptides mixed in adjuvant exhibit CNS demyelination. Furthermore, free radicals activate transcription factors resulting in the up-regulation of the expression of many genes that are associated with MS such as NF-k- $\beta$, TNF- $\alpha$, intracellular adhesion molecule 1 (ICAM-1), and vascularcell adhesion molecule 1 (VCAM-1). ${ }^{45,68}$ A study by Langemann et al., revealed that MS plaques had increased free radical activity as well as decreased levels of important antioxidants such as glutathione, alpha-tocopherol, and uric acid. ${ }^{69}$ Further evidence has shown that oxidative damage to DNA in MS includes damage to mitochondrial DNA, implicating mitochondria not only in the formation of ROS but possibly as a pathway directly affected by OS due to the formation of ROS. ${ }^{70}$ Studies have also shown that nitric oxide synthase (NOS) is upregulated in inflammatory lesions and that $\mathrm{NO}$ and its derivative peroxynitrite inhibit mitochondrial respiration. ${ }^{6,66,71} \mathrm{NO}$ is both essential for life and toxic. Its immunomodulatory effect helps sustain healthy homeostasis; however, large NO quantities damage axons. ${ }^{6}$ Inflammation induces the production of NO. Excessive generation of $\mathrm{NO}$ is an indicator of aging and neurodegeneration. Increased NO concentration raises intracellular $\mathrm{Ca}^{2+}$ and $\mathrm{Na}^{+}$levels and may be responsible for mitochondrial dysfunction. ${ }^{6,72}$ Tissue damage in MS is caused in part by elevated levels of NO. In the CNS, NO is produced by macrophages and microglia following the induction of nitric oxide synthase (NOS) by the proinflammatory cytokines TNF- $\alpha$ and INF- $\gamma .^{73}$ Notably, NO has been show to prevent the stabilization of HIF through an increase in prolyl hydroxylase dependent mechanism leading to HIF degradation. ${ }^{74,75}$ Another oxidative agent, $\mathrm{H}_{2} \mathrm{O}_{2}$, decreased HIF-DNA binding capacity and the expression of its target genes. ${ }^{76,77}$ Taken together these studies suggest that redox system plays an important role in HIF regulation.

NO can also react with sulfhydryl groups of proteins resulting in S-nitrosylation of target proteins, which initially can act as a protective mechanism in OS to defend proteins from degradation. However, increased OS and over accumulation of NO, results in irreversible cell damage caused by oxidation of free thiols, nitration of tyrosine residues, and lipid peroxidation. ${ }^{78}$ Increased S-nitrosylation has been detected in normal appearing white matter of MS patients' brain compared to normal controls, indicating that nitrosative damage is involved in the pathophysiology of MS. ${ }^{79}$

In addition to ROS, glutamate appears to be another major source of OS in the brain through the activation of ionotropic glutamate receptors. It is possible that damage induced by free radicals can occur by the stimulation of phospholipase A2 (PLA2) and 
the release of amino acids, which in the presence of free radicals results in an enhanced release of glutamate. ${ }^{80,81}$ Cerebrospinal fluid (CSF) of MS patients has elevated levels of glutamate. ${ }^{82,83}$ Increased glutamate via interaction with alpha-amino-3-hydroxy-5methyl-4-isoxazolepropionic acid (AMPA)/kainate receptors have also shown to be deleterious to oligodendrocytes (which appear to be highly vulnerable to glutamate excitotoxicity). ${ }^{84,85}$ Interestingly, AMPA/kainate receptors are known to have increased permeability to $\mathrm{Ca} 2+$ resulting in $\mathrm{Ca} 2+$ loading of cells. ${ }^{86}$ As the oxidative stress theory stands, it appears that ROS in the presence of a weakened antioxidant cellular defense results in the damage of cellular components such as lipids, proteins, nucleic acids and mitochondrial DNA. These damaged components alter multiple pathways, associated with ATP production, up regulation of genes associated with MS pathology, and increase glutamate levels (via AMPA/kainate receptors).

\section{Molecular Mimicry}

Molecular mimicry is one mechanism that explains the association between an autoimmune response to an environmental agent acting as a contributor to the pathogenesis of MS. ${ }^{87-92}$ The hypothesis of molecular mimicry describes the molecular consequences of immunological cross reactivity: when T-cells or antibodies specific for an environmental agent (such as a virus) cross react with a host antigen, resulting in target cell and organ damage. Previously it has been shown that patients with HTLV-1 Associated Myelopathy/Tropical Spastic Paraparesis (HAM/TSP), an HTLV-1 virus induced spastic paraparesis clinically similar to MS, produce autoantibodies to the RNA binding protein hnRNP A1. ${ }^{93}$ Similary, it has been presented that patients with MS produce antibodies to hnRNP A1, specifically to the M9 region of the protein. ${ }^{94}$ M9 serves as the nuclear export sequence/nuclear localization sequence for hnRNP A1. 1,2,11,12,93-99 Anti-hnRNP A1 antibodies have been shown to produce deleterious effects upon neuronal cells. It has been shown that anti-hnRNP A1 antibodies reduce cellular ATP, increase apoptosis, reduce neuronal firing, and cause a redistribution of endogenous hnRNP A1 protein. ${ }^{1,94}$ Furthermore, transfection of anti-hnRNP A1 antibodies into neurons caused changes in transcripts related to A1 function, specifically the spinal paraplegia genes (SPGs). ${ }^{12}$ These findings of altered RNA levels of the SPGs were further confirmed in IgG isolated from MS patients compared to controls. ${ }^{12}$ Additionally, we present data supporting anti-hnRNP A1 antibodies role in a "dying back" neurodegenerative mechanism in vivo in a murine EAE model of MS. In summary, patients with MS produce autoantibodies to the RNA binding protein hnRNP A1 similar to patients with HAM/TSP. Anti-hnRNP A1 antibodies appear to cause multiple deleterious effects both in vitro and in vivo which could provide a possible mechanism for the neurodegeneration seen in patients with MS.

\section{Antibodies}

Antibodies present in MS patients can be categorized into two major groups depending on their target antigens: myelin and non-myelin antibodies. In vitro and in 
vivo studies suggest that both types of antibodies have some involvement in the pathogenesis of MS. ${ }^{11}$ Anti-myelin antibody targets include myelin oligodendrocyte glycoprotein (MOG), myelin basic protein (MBP), myelin-associated glycoprotein (MAG), and proteolipid protein (PLP). ${ }^{100-103}$ These antibodies have been found in sera and CSF of MS patients, however the exact role of such myelin antigens in MS remains contradictory. One study demonstrated that development of clinically definite MS could be predicted based on the presence of anti-MOG and anti-MBP antibodies in patient's sera; others found no association between anti-MOG and anti-MBP antibodies and MS progression. ${ }^{104-106}$ Immunopathogenic effects of anti-myelin antibodies might be epitope specific or depend on the antibody confirmation. ${ }^{102,107-109}$ Early studies did not specify cellular pathways affected by autoantibodies, however in a recent study Ho et al., showed that MAG autoantibodies targeted the following natural brain lipids: 1-palmitoyl-2glutarol-sn-glycero-3-phosphocholine (PGPC), 1-palmitoyl-2-oleoyl-sn-glycero-3(phospho-L-serine), (POPS), 1-hexadecyl-2-azelaoyl-sn-glycero-3-phosphocholine (azPC) and 1-palmitoyl-2-azelaoyl-sn-glycero-3-phosphocholine (azPC ester). ${ }^{110}$ Moreover the authors showed that that POPS, PGPC, azPC, and azPC ester affected inflammatory, survival, and apoptotic signaling pathways. Specifically, canonical NF-k- $\beta$ and extracellular signal-regulated kinase (ERK) pathways were activated in stimulated Tcells isolated from EAE mice. Overall their data suggests that myelin phospholipids are targeted by autoimmune responses in MS.

A growing number of studies point to the fact that antibody-mediated axonal injury could be initiated by antibodies to non-myelin antigens. Non-myelin antibodies present in MS patients target neuronal surface molecules (axolemma-enriched fractions, neurofascin, gangliosides), cytoskeletal proteins (neurofilaments (NF), neurofilament light chain NF-L), intracellular enzymes, signaling molecules and chaperones ( $ß$-arrestin, retinal arrestin, heat shock proteins, glutamate decarboxylase, proteasomes) and nuclear antigens (nuclear ribonuclear proteins). ${ }^{111}$ These antibodies present a different mechanism of immune-mediated axonal injury. For example, antibodies to neurofascin (NF-186, NF-155), which is a cell adhesion molecule involved in axon subcellular targeting and synapse formation during neural development, caused exacerbation of EAE without demyelination in the spinal cord of rats. When anti-neurofascin antibodies were co-transfected with MOG specific T-cells, they selectively targeted Nodes of Ranvier and inhibited neurotransmission in an MS animal model. ${ }^{12}$ In separate experiments, chronic progressive MS patients had significantly higher levels of NF-L specific antibodies in sera compared to that of patients with other neurological diseases. ${ }^{113,114}$ How the anti-NF and anti-NF-L antibodies arise and what their specific effects are on the axon is unclear, but their correlation with disease progression appears applicable in a biomarker specific manner. ${ }^{112-114}$

In addition, antibodies to hnRNP A1, B2 (hnRNP A1/B2) were present in CSF of MS and HTLV-1 associated myelopathy/tropical spastic paraparesis (HAM/TSP) patients, but not in normal controls. ${ }^{12,92-94,97,98,115}$ hnRNPs are RNA-binding proteins that play a major role in adjustment of pre-mRNA splicing through various factors. ${ }^{116}$ They also participate in mRNA stability, NF-k- $\beta$ dependent transcription and telomerase activity. ${ }^{117-119}$ hnRNP A1 plays several key roles in neuronal functioning and its 
depletion, either due to debilitated cholinergic neurotransmission or causing drastic changes in RNA metabolism during autoimmune reactions. ${ }^{12,120}$ Recently, RNA binding proteins have gained attention because a large number of these proteins were mutated in neurodegenerative diseases. ${ }^{121,122}$ RNA binding proteins use protein aggregation as part of a normal regulated, physiological mechanism that controls protein synthesis. The process of regulated protein aggregation is most evident in the formation of stress granules (SG). ${ }^{123}$ HnRNPA1 has been shown to relocate into cytoplasmic SG in the presence of stress stimuli, such as osmotic shock or OS. ${ }^{124}$ Further, studies showed that addition of antibodies to hnRNPA1 affected its distribution from a primarily nuclear location to a mixed nuclear/cytoplasmic distribution. ${ }^{12,95,125}$ It was known that antihnRNP A1 antibodies decreased neuronal firing in vitro but it wasn't clear if the antibodies to this intracellular protein could penetrate neurons and find its target. ${ }^{94}$ Recent studies revealed that anti-hnRNP A1 antibodies penetrated neuronal cells via clathrin mediated endocytosis and caused deleterious effects to the neurons. ${ }^{12,94,95,125}$ Specifically, anti-hnRNP A1 antibodies increased apoptosis, reduced ATP levels, and caused redistribution of endogenous hnRNPA1 protein. Thus, anti-hnRNP A1 antibodies altered endogenous protein localization as well as inhibited normal cellular processes in vitro.

The presence in MS patients of two types of autoantibodies may not be mutually exclusive. It is possible that antibodies to myelin antigens may have an impact on the early, relapsing stages of disease while the non-myelin antigens play a more dominant role in progressive stages of MS. More importantly, both types of antibodies may cause neurodegeneration through activation of apoptotic, inflammatory cytokine, and immune response pathways.

\section{hnRNP A1 and M9 Antibodies}

hnRNP A1 is an RNA binding protein overexpressed in neurons. hnRNP A1 is a member of a family of over 20 nuclear proteins designated as heterogeneous nuclear ribonucleoproteins (hnRNPs). ${ }^{126}$ The hnRNPs are involved in pre-MRNA processing events as well as transport of mRNAs to the cytoplasm for translation in eukaryotic cells. ${ }^{126}$ While some hnRNPs are strictly nuclear molecules, a subset of hnRNPs, including hnRNP A1 shuttle constantly between the nucleus and the cytoplasm. ${ }^{126}$ hnRNP A1 functions in pre-mRNA packaging, splicing, mRNA metabolism and transport. ${ }^{126,127}$ hnRNP A1 is a 320 amino acid protein with many functional motifs. ${ }^{127}$ The N-terminus of hnRNP A1 consists of 185 residues which can be divided into two 90 amino acid repeats which function as RNA recognition motifs (RRM) or RNA binding domains (RBD). ${ }^{127}$ Within each RBD are two consensus binding sequences termed RNP1

and RNP2 which are part of the binding surface of the RBD. ${ }^{127}$ The remaining 135 amino acid $\mathrm{C}$ terminal region contains additional functional motifs. The $\mathrm{C}$-terminal region is rich in glycines and contains an RGG box region that is involved in RNA binding, protein-protein interactions, nuclear localization, and RNA strand annealing activity. ${ }^{127}$ Near the end of the C-terminus of hnRNP A1 is the M9 sequence encompassing amino acids 268-305. M9 serves as both a nuclear export sequence and nuclear localization 
sequence (NES/NLS) for hnRNP A1, thus making this region responsible for the nuclear/cytoplasmic shuttling of the hnRNP A1 protein. ${ }^{126,128}$ The final motif on the Cterminus of hnRNP A1 is termed the ' $\mathrm{F}$ peptide'. The F peptide spans the region from amino acids 301-319 and must be phosphorylated to initiate the binding of hnRNP A1 to transportin 1 (Trn1) which is necessary for cellular trafficking between the nucleus and cytoplasm. $^{129}$

Mechanisms involving RNA metabolism, and in particular RBPs, are becoming increasingly important in the pathogenesis of neurodegenerative disorders such as frontotemporal dementia, multisystem proetinopathy, and amyotrophic lateral sclerosis (ALS). ${ }^{130-132}$ Importantly, MS patients have been shown to develop autoantibodies to the RBP hnRNP A1. ${ }^{11,12,92,94,95,97,98,125}$ Epitope analyses has shown that the antibody response in MS patients is specific for the 'M9' region of hnRNP A1. M9 is hnRNP A1's nuclear export sequence/nuclear localization sequence (NES/NLS) which is required for hnRNP A1's transport into and out of the nucleus. ${ }^{12,92-95,97,98,125,126}$ Previous studies have shown that anti-hnRNP A1-M9 antibodies transfected into neuronal cells cause neurodegeneration and alter the RNA expression of the spinal paraplegia genes (SPG4 (spastin), SPG7 (paraplegin), and SPG20 (spartin)). ${ }^{12,125,126}$ Mutations in the SPGs (of which spastin is the most common) result in hereditary spastic paraparesis (HSP), which is clinically indistinguishable from progressive MS. ${ }^{12,126,133}$ It appears there may be a potential link between anti-hnRNP A1, M9 specific antibodies, produced in MS patients and the alterations in the SPGs expression levels which could be a possible contributing factor to the neurodegeneration seen in MS.

\section{Apoptosis}

Apoptosis is the term utilized to describe the process of programmed cellular death. ${ }^{134}$ Apoptosis is a vital component of numerous cellular processes including development and function of the immune system, cell turnover, embryonic development, and naturally induced cellular death by humoral and chemical systems within the body. ${ }^{134}$ During development and aging, apoptosis occurs normally within the body as a homeostatic mechanism in order to maintain tissues and cell populations. ${ }^{134}$ Through a protective manner apoptosis can act as an immune response by causing cell death in cells that have been attacked by a toxic foreign agent. ${ }^{134}$

There are two main pathways leading to apoptosis: the extrinsic "death receptor" and intrinsic or "mitochondrial" pathway. ${ }^{134}$ Although once thought to be completely separate pathways, evidence now suggests that molecules in one pathway can have effect on the other pathway. ${ }^{134} \mathrm{~A}$ third pathway has emerged involving apoptosis induced by cytotoxic T-cells through what is now called the perforin/granzyme pathway. ${ }^{134}$ Although each pathway is initiated in a different fashion, each pathway shares the same executioner caspase, caspase $3 .{ }^{134}$ The executioner pathway is initiated by the cleavage of caspase $3{ }^{134}$ The action of caspase 3 cleavage results in DNA fragmentation, nuclear and cytoskeletal protein degradation, apoptotic body formation, protein cross-linking, phagocytic cell receptor expression, and ultimately phagocytosis of the cell. ${ }^{134}$ Two of the 
main mechanisms used to determine apoptotic events within studies is to measure the ATP levels and cleaved caspase 3 levels of cells.

\section{Endosomes/ Clathrin and Caveolar Mediated Endocytosis}

Endocytosis is the invagination of the plasma membrane and the formation of vesicles and vacuoles through membrane fusion which internalizes fluid, solutes, macromolecules, and other cellular components. ${ }^{135}$ The two main types of endocytosis are clathrin-mediated endocytosis and caveolar-mediated endocytosis. ${ }^{135}$ Caveolar uptake is performed by non-coated plasma membrane invaginantions called caveolae. ${ }^{136}$ Caveolae are notably known for their association with caveolins which are a family of cholesterol-binding proteins. ${ }^{136}$ Caveolins are responsible for maintaining the structure of the endosomes formed during caveolar-mediated endocytosis. ${ }^{136}$ Notably, many of the molecules within caveolae are also present within lipid rafts. ${ }^{136}$ Clathrin-mediated endocytosis takes place when specific molecules are taken up into clathrin coated invaginations for transport within the cell. ${ }^{137}$ The adaptor complex AP2 is a multimeric protein on the plasma membrane responsible for internalizing molecules into the clathrincoated vesicles for transport. ${ }^{137}$ Once selective cargoes are packaged within the clathrin vesicles, these transport vesicles fuse with early endosomes for transport. ${ }^{137}$ Depending on cargo being assessed, molecules can be transported by one or both of these two pathways.

Within neurons, endosomes play a vital role in maintaining viability of the cell due to their role in transporting various molecules throughout the axon. It has been widely accepted that signaling endosomes are the transport mechanism utilized by these essential molecules for transport anterogradely as well as retrogradely along microtubules in the axon. ${ }^{138}$ The motors responsible for axonal transport along microtubules are dyneins and kinesins. ${ }^{138}$ Dynein motors are responsible for retrograde transport while kinesins are the anterograde motors. ${ }^{138}$ Both kinesin and dynein motors transport long range signals along the axon between the soma (cell body) and the axon terminals. ${ }^{138}$ One example of retrograde signaling can be seen through the internalization of Trk receptors via clathrin-mediated endocytosis into signaling endosomes at the distal axon. These internalized Trk receptors are then retrogradely transported to the soma in order to initiate survival responses. ${ }^{138}$ Another example of signaling endosomes can be visualized through the transport of La, an RBP. La protein is an RBP that is anterogradely transported along microtubules via kinesin motors. ${ }^{138}$ When La reaches its destination at the axon terminal it is covalently modified by small ubiquitin-like modifying polypeptides (SUMO). ${ }^{138}$ Once 'sumoylated', La is then able to bind dynein motors and transport retrogradely back to the soma, thus finishing its transport cycle. ${ }^{138}$ Studies have shown that the disruption of retrograde transport leads to a variety of neurodegenerative disorders. ${ }^{138}$ Diseases such as hereditary spastic paraplegia (HSP) and ALS are characterized by "dying back" of axons in which the axon degeneration shows a distal to proximal progression. ${ }^{138}$ Specifically, the disruption of dynein motor function has been shown to cause neurodegeneration in both mouse and human diseases. ${ }^{138}$ However, it also appears that anterograde transport could in theory have an effect on 
neurodegeneration. It has been shown that disruption of retrograde or anterograde transport can cause a blockage of trafficking in both directions due to a resulting "traffic jam". ${ }^{138}$ This traffic jam, which can be caused by disruption of either transport mechanism results in the impaired transport of signaling endosomes. ${ }^{138}$

\section{RNA Granules}

Neurons are required to communicate over long distances in a bidirectional manner. This bidirectional communication system utilizes macromolecules encoded with essential information which link synapses, dendrites, axons, and the soma. ${ }^{139}$ Bidirectional transport is composed of two different transport systems, anterograde and retrograde transport. Anterogradely, messenger RNA (mRNA) is transported from the soma to the terminals of the axon. The mRNA is locally translated at the terminals. This new translated protein is then shipped back to the soma by retrograde transport. Anterograde and retrograde transport use slightly different mechanisms of action. Anterograde transport occurs due to the transport of RNA granules with the use of kinesins as the motor to transport the molecules anterogradely down microtubules toward the axon terminals until they reach sites of translation prior to reaching axon terminals in most instances. ${ }^{139}$ Retrograde transport uses signaling endosomes as well as importins and dynein to transport molecules retrogradely along microtubules from axon terminals back to the soma. ${ }^{139}$ Signaling endosomes are the results of neutrophins (neuronal growth factors) being released from target tissues and binding to Trk receptors. This binding action forms a ligand- receptor complex, which is internalized forming a signaling endosome. Importins and dynein are proteins that act as the motor during retrograde transport. When importins and dynein are bound directly by molecules such as signaling endosomes, the importins and dynein act as a motor by trafficking the bound molecule in a retrograde fashion along the microtubule network towards the soma. ${ }^{139}$

RNA granules also known as neuronal granules are utilized in anterograde transport and consist of RNA binding proteins, mRNAs, ribosomal machinery for translation of mRNA, and microtubule motors. ${ }^{140}$ RNA granules (neuronal granules) are utilized to transport mRNA in an anterograde manner along the microtubules for localized translation likely near the axon terminals. ${ }^{140,141}$ It is suggested that there may be multiple types of RNA granules due to a lack of complete co-localization of various proteins and RNA granule markers. ${ }^{140}$ Additionally, the mechanism by which ribosomal subunits within the RNA granules are repressed from translating mRNAs within the granules is not clearly understood. However, it is hypothesized that something within the RNA granules, such as micro-RNAs may act to block the ribosomal subunits by blocking mRNA elongation at the polysome stage. ${ }^{140}$

In addition to the role RNA granules play in neuronal trafficking under normal conditions, they also play a very important role in neurons during stress. In response to stress stimuli, RNA granules form two main types of stress induced granules known as Processing bodies (P bodies) and stress granules. ${ }^{124} \mathrm{P}$ bodies are RNA granules whose internal mRNA cargo is tagged for degradation. ${ }^{124,141}$ Stress granules are RNA granules, 
which form during nutrient deprivation or metabolic stress and contain mRNA cargo that is stored in an abortive transition initiation complex enabling them to enter rapid reinitiation once stress stimuli are gone. ${ }^{124}$

RNA binding proteins (RBPs), which bind and transport mRNA, are responsible for the recruitment of themselves as well as their bound cargo to RNA granules. There are several RBPs present within RNA granules. Each RBP contains a LC region (low complexity polypeptide sequence) within the RBPs with little diversity in their amino acid composition. ${ }^{141}$ The LC region is composed of many tyrosine residues and is responsible for the localization of the RBP-mRNA complex to RNA granules. hnRNP A1 is one of the RBPs found within RNA granules during the stress response. ${ }^{124,140,141}$ hnRNP A1 binds various mRNAs for transport and translation within neurons. hnRNP A1 contains an LC region between amino acids 186-320 which includes the M9 region (268-305) and the F peptide (305-320). ${ }^{141}$ During stress, a stretch of serines located in the F peptide is phosphorylated by Mnk $1 / 2$ kinase. ${ }^{124}$ The phosphorylation of the F peptide results in a redistribution of hnRNP A1 to the cytoplasm by abrogating hnRNP A1 and TNPO1 interactions. ${ }^{124} \mathrm{hnRNP}$ A 1 and its bound mRNA cargo localize to stress granules but are not found within $\mathrm{P}$ bodies during the stress response. ${ }^{124}$ The action of hnRNP A1 binding to stress granules rather than P bodies, provides hnRNP A1 and its bound mRNA cargo a harbor of safety until the stress response has been abolished at which point hnRNP A1 is released from the protective stress granules where it can continue trafficking its mRNA cargoes. Interestingly, hnRNP A1 binding to poly(A) mRNA increases during the presence of stress whereas its binding to TNF- $\alpha$ mRNA decreases during the presence of stress. ${ }^{124}$ It appears that hnRNP A1 shows a preference to protect poly(A) mRNA over TNF- $\alpha$ mRNA during stress.

\section{Experimental Autoimmune Encephalomyelitis (EAE)}

EAE is a mouse model of CNS autoimmune disease mediated by $\mathrm{CD}^{+}{ }^{+} \mathrm{T}_{\text {-cells. }}{ }^{142}$ The disease is exemplified by demyelination of axons caused by perivascular $\mathrm{CD} 4^{+} \mathrm{T}$ cells and mononuclear infiltration leading to hind-limb paralysis. ${ }^{142,143}$ Serving as the best animal model to date for MS, EAE mice are shown to have similar disease course and features as MS patients such as inflammation, demyelination, axonal loss, and gliosis. ${ }^{143}$ EAE provides a relevant model to study the immune response as well as axonal damage in the immune-mediated demyelinating disorder MS. ${ }^{142,143}$ There are two methods of EAE disease induction: the active induction and adoptive transfer model. ${ }^{142}$ Both methods can be used in female SJL or C57Bl/6 mice, however the disease course between the two strains varies slightly. SJL mice tend to produce a relapsing-remitting disease course, whereas the C57/B16 mice produce a more chronic and continuous disease. $^{143}$

The active induction method is often termed the "classic" EAE model. This model is induced by immunization with myelin peptides such as proteolipid protein (PLP), myelin oligodendrocyte glycoprotein (MOG), or myelin basic protein (MBP) in complete Freund's adjuvant (CFA). ${ }^{12,143}$ The immunization is performed concurrently 
with pertussis toxin injection to disrupt the blood-brain barrier BBB. In the active induction model, the myelin peptide of choice often depends on mouse strain and symptomology desired. SJL mice are regularly immunized with PLP and demonstrate a relapsing-remitting disease course. ${ }^{143} \mathrm{C} 57 \mathrm{Bl} / 6$ mice are generally immunized with MOG and display a more chronic, "smoldering" disease course. ${ }^{143}$ The disease onset generally occurs within 9-12 days post immunization and disease course varies as mentioned previously. ${ }^{143}$

The passive or "adoptive transfer" model utilizes passive transfer of encephalogenic $\mathrm{CD} 4^{+} \mathrm{T}$-cells into recipient mice. ${ }^{142,143}$ In this model, donor mice are initially immunized as in the active induction model, however, these donor mice are not injected with pertussis toxin. ${ }^{142}$ Approximately 10 days following immunization of donor mice, spleens and lymph nodes are extracted and T-cells are cultured for 3-5 days in media containing a milieu of cytokines to produce encephalogenic $\mathrm{CD} 4^{+}$T-cells. $^{142}$ Following culture of the "activated" $T$ cells, recipient mice are injected with the encephalogenic $\mathrm{CD}^{+} \mathrm{T}$-cells by either i.p or i.v. to induce disease. The adoptive transfer model can also be induced in either SJL or $\mathrm{C} 57 \mathrm{Bl} / 6$ mice with slightly different disease course similar to that described in the active induction model. ${ }^{142}$ The disease course of adoptive transfer model mice is overall generally more severe than the disease course of active transfer model mice. ${ }^{142}$ The adoptive transfer model mice generally display symptoms within 6-10 days post transfer.

Clinical scoring of EAE is based on a scale from 0-5 to most accurately mimic the human disease based on the symptoms exhibited by mice. The basic scoring system is as follows: 0- normal mouse with no disease symptoms; 1- limp tail or hind limb weakness, but not both; 2- limp tail and hind limb weakness; 3- partial hind limb paralysis; 4complete hind limb paralysis; 5- moribund state, death. ${ }^{142}$ Within the scoring system there are also 0.5 increments based on certain visualized occurrences that would place the animal between scoring levels.

\section{Antibody Augmented EAE}

Within the world of autoimmune disorders, autoantibodies to specific antigens have been implicated to show specificity to certain disorders. For example, patients with MS and HAM/TSP have been shown to produce autoantibodies to the M9 epitope of the protein hnRNP A1. ${ }^{11,12,92-95,97,98,125}$ Other autoimmune disorders have also implicated various autoantibodies specific to certain diseases. Anti-recoverin antibodies were found to be present in patients with cancer associated retinopathy (CAR). ${ }^{144}$ Interestingly it was discovered that anti-recoverin antibodies lead to apoptosis of retinal cells in CAR. ${ }^{144}$ In patients with neuromyelitis optica (NMO), patients produce antibodies to aquaporin4. ${ }^{19,145}$ Bennett et al. demonstrated that six different recombinant antibodies produced which were specific to aquaporin-4 epitopes contributed to CNS pathology in Lewis rats with EAE. ${ }^{146}$ Furthermore, Bradl et al. treated EAE Lewis rats with IgG isolated from both antibody-positive and antibody negative NMO patients. Bradl et al. provide evidence that while antibody-negative patient IgG have little effect to augment disease, 
antibody-positive IgG from NMO patients worsen disease and induce NMO-like lesions in Lewis rats with EAE. ${ }^{145}$

\section{Objectives and Specific Aims}

\section{Objectives}

At the onset of my project, it was previously shown that patients with MS produced autoantibodies against the RBP hnRNP A1. Data at this point also demonstrated that the autoantibodies contained epitope specificity to hnRNP A1-'M9' (hnRNPA1's NES/NLS). Additionally, it had been shown that anti-M9 specific antibodies decreased neuronal firing in patch-clamp experiments and caused neurodegeneration in a neuronal cell line.

My project was designed to further determine the effect of anti-M9 antibodies on neuronal cells both in vivo and in vitro. My project objectives included determining mechanisms of antibody entry into neuronal cells, anti-M9 effects on cellular viability, neuronal protein expression and RNA levels, and to determine antibody effect upon the in vivo EAE murine model of MS. Additionally, my project included aims to further the understanding of mRNA binding partners of hnRNP A1 as well as determine whether anti-M9 antibodies have an effect on hnRNP A1 and RNA granules during cellular stress.

\section{Specific Aim 1}

Describe anti-hnRNPA1 antibodies penetration and the deleterious effects they have on neuronal cells in vitro.

\section{Specific Aim 2}

Analyze whether a hnRNP A1 protein - spastin mRNA interaction exists as well as determine whether anti-hnRNP A1-M9 antibodies possess the ability to interact with RNA granules.

\section{Specific Aim 3}

Determine the effects of anti-hnRNP A1-M9 antibodies on the clinical phenotype and cellular mechanisms of neurodegeneration in the CNS of mice with EAE. 


\title{
CHAPTER 2. MATERIALS AND METHODS
}

\author{
Aim 1 Methods
}

\section{Cells}

SK-N-SH cells (ATCC, HTB-11), an immortalized human neuroblastoma cell line was maintained in Dulbecco's modified Eagle's medium (DMEM) with F12, supplemented with $10 \%$ fetal bovine serum and $1 \%$ penicillin-streptomycin antibiotics.

\section{Antibodies}

Anti-hnRNP A1 antibodies specific to the M9 epitope of hnRNP A1 were obtained from Abcam (ab 4791-rabbit polyclonal, ab 5832- mouse monoclonal) and rabbit IgG were obtained from Abcam (ab 107866 ) and Millipore ( 12-370). Anticlathrin antibodies were purchased from Novus Biologicals (NBP1-05991).

\section{Atto550 NHS Labeling of Anti-hnRNP A1 and Normal IgG Antibodies}

A $0.1 \mathrm{M} \mathrm{NaHCO} 3$ buffer was made $(8.4 \mathrm{~g} \mathrm{NaHCO} 3,22.9 \mathrm{~g} \mathrm{NaCl}, 1$ liter $\mathrm{H} 2 \mathrm{O})$ and brought to a $\mathrm{pH}$ of 8.4 with the following solution: $10.6 \mathrm{~g} \mathrm{Na} 2 \mathrm{CO} 3,29.2 \mathrm{~g} \mathrm{NaCl}, 1$ liter H2O. $35 \mu \mathrm{g}$ of antibodies $(1 \mu \mathrm{g} / \mathrm{ml})$ were added to $70 \mu \mathrm{l}$ of Atto550 NHS Ester (SigmaAldrich, 92835) and $500 \mu \mathrm{l}$ of $\mathrm{NaHCO} 3$ buffer. The mixture was rotated for 1 hour at room temperature. After the hour-long incubation, the mixture was injected into Slide-alyzer dialysis cassette (ThermoFisher Scientific) and dialyzed overnight in PBS. The next day the labeled antibodies were concentrated by centrifugation in Amicon filters (Millipore) and concentration was determined with a NanoDrop 1000 spectrophotometer (ThermoFisher Scientific).

\section{Antibody Penetration Tracing}

150,000 SK-N-SH cells were seeded per chamber into four chamber slides. Atto550 labeled anti-hnRNP A1 and rabbit IgG were added at a concentration of $2 \mu \mathrm{g} / \mathrm{ml}$ directly into DMEM. An untouched control cell group was employed as a negative control. At 24 and 72 hours after antibody and $\operatorname{IgG}$ addition, cells were fixed with 4\% paraformaldehyde for 15 minutes at room temperature. Cells were then washed 4 times 5 min each with PBS, mounted, and imaged using a Zeiss Axio Observer A1 fluorescent microscope (Carl Zeiss). Quantification of antibody penetration percentage between the anti-hnRNP A1 treated and control rabbit IgG treated cells was performed using AxioVision Software. Five representative images were used per treatment from the 24 hour images. Antibody penetrated cells were divided by the total number of cells to give 
a percentage of antibody penetration: Cells with antibodies per image/Total cells per image) $\times 100=$ Penetration Percentage).

\section{Anti-hnRNP A1 and Early Endosomes Co-localization}

100,000 SK-N-SH cells per chamber were seeded into 8 chamber slides. Atto550 labeled antibodies were added directly into DMEM/F12 at a concentration of $2 \mu \mathrm{g} / \mathrm{ml}$. At 24 hours prior to antibody addition, $20 \mu 1$ of Cell Light Early Endosome GFP (Invitrogen) was added to label endosomes. At 6, 24, and 48 hours after antibody addition, cells were fixed with 4\% paraformaldehyde for 15 minutes at room temperature. Cells were then washed, mounted, and observed as described above. Quantification of the endosome penetration for the three time points was performed in AxioVision Software Version 4.8. Total colocalization events per cell was determined for 5 representative cells at the $6 \mathrm{hr}$., $24 \mathrm{hr}$, and $48 \mathrm{hr}$. time points respectively.

\section{Determination of Clathrin or Caveolar Mediated Endocytosis}

2.5 million SK-N-SH cells were seeded into 35mm glass-bottom dishes (MatTek Corp). Three plates each were designated as clathrin or caveolar mediated endocytosis. Pitstop 2 reagent (Cellagen tech, C7487-25) was added at a 10nM concentration for 30 minutes into DMEM/F12 media to block clathrin mediated endocytosis. Nystatin $(24 \mu 1 / \mathrm{mL})$ solution (Sigma-Aldrich, N1638) was added into three separate dishes for 30 minutes to block caveolar mediated endocytosis. After the 30-minute incubation, antihnRNP A1 and IgG antibodies were added at a concentration of $4 \mu \mathrm{g} / \mathrm{mL}$. Live imaging of the cells was performed at 6,12, and 24 hours after antibody addition in order to determine antibody penetration. Co-immunoprecipitations were performed in order to confirm the clathrin pathway as the method of penetration. Protein A beads were blocked with 6\% Milk-TBS for an hour at room temperature. Beads were then washed $4 \times 5$ minutes with IP buffer. Anti-hnRNP A1 antibodies and control rabbit IgG were attached to protein A beads overnight at 4C. Beads were then washed $4 \times 5$ min with IP buffer. SK-N-SH cell lysate was added over beads without antibodies, hnRNP A1 antibody beads, and control IgG beads overnight at 4C. Beads were washed 4 x 5 minutes with IP buffer. Beads were spun down 5 minutes at $5000 \mathrm{rpm}$. $50 \mu \mathrm{l}$ of $2 \mathrm{x}$ buffer was added per tube and heated 5 minutes at $95 \mathrm{C}$. Tubes were then cooled while spinning $5000 \mathrm{rpm}$ for 5 min at $4 \mathrm{C}$. $150 \mu \mathrm{g}$ of each sample was loaded onto a $10 \%$ Tris gel and run at $100 \mathrm{~V}$ for 90 minutes. The gel was then transferred to a PVDF membrane at $100 \mathrm{~V}$ for 90 minutes. The membrane was blocked for 30 minutes at room temperature with $6 \%$ milk-TBS. Membranes were then washed 4 x 5 minutes with TBS-T. Anti-clathrin interactor-1 antibodies (NBP1-05991) were added at a concentration of 1:2000 in 6\% milk-TBS overnight at $4 \mathrm{C}$. The membrane was then washed 4 x 5 minutes in TBS-T. Secondary anti-rabbit HRP antibodies were added for 50 minutes at room temperature at a concentration of 1:10000. The membrane was then washed 4 x 5 minutes. SuperSignal West Pico Chemiluminescent Substrate (ThermoFisher Scientific) was then added for 5 minutes and the membrane was immediately imaged. 


\section{Measurement of ATP Levels}

ATP levels were determined using the CellTiter-Glo Luminescent Cell Viability Assay (Promega) which quantitates the ATP levels based upon luminescence signal production which is equivalent to total ATP present. The CellTiter-Glo system utilizes a luciferin based assay which in the presence of ATP converts luciferin to oxyluciferin, resulting in the production of a luminescent signal. The intensity of the luminescence (RLU=Relative Luminescent Units) is directly proportional to the amount of ATP within the system. 50,000 SK-N-SH cells were seeded per well into a 96 well plate. AntihnRNP A1 antibodies produced in rabbit and normal rabbit IgGs were added at a concentration of $8 \mu \mathrm{g} / \mathrm{ml}$ directly into $100 \mu \mathrm{l}$ of DMEM/F12 media. An untouched control cell group also served as a control. At 48 and 72-hour points after antibody addition $100 \mu \mathrm{L}$ of CellTiter-Glo Reagent (Promega) was added. After one hour of incubation, the plate was read on a spectrophotometer between wavelengths $560 \mathrm{Ex} / 590 \mathrm{Em}$.

\section{Determination of Antibodies Effect on Caspase 3/7 Levels}

50,000 SK-N-SH cells were seeded per well into a 96 well plate. Anti-hnRNP A1 antibodies and normal rabbit IgGs were added at a concentration of $8 \mu \mathrm{g} / \mathrm{ml}$ and an untouched control cell group served as an additional control. At 24, 48, and 72 hours after antibody addition $100 \mu 1$ of ApoOne Homogenous Caspase 3/7 reagent (Promega) was added per well. After one hour of incubation the plate was read on a spectrophotometer at $485 \mathrm{Ex} / 530 \mathrm{Em}$ wavelengths.

\section{Measurement of Cytotoxicity}

Cytotoxicity was determined using the Cytotox-ONE Cytotoxicity Assay (Promega). Cytotox-ONE Cytotoxicity Assay determines cytotoxicity of the cell by determining membrane permeability by measuring the release of lactase dehydrogenase (LDH) from the cell. This is measured by the LDH dependent conversion of resazurin into the fluorescent resarufin product. The amount of cytotoxicity is measured as relative fluorescent units (RFU). 100,000 SK-N-SH cells were seeded per well in triplicates into a 96 well plate. Anti-hnRNPA1 antibodies and normal rabbit IgGs were added at a concentration of $8 \mu \mathrm{g} / \mathrm{ml}$ and an untouched control cell group served as normal cytotoxicity percent control and a group with only culture media served as a background control. At 24, 48, and 72 hours the plate was taken out of the incubator and lysis solution (Promega) was added for 30 minutes at room temperature. Cytotox-ONE reagent was then added to all chambers and let to sit at room temperature for 10 minutes. Stop solution was then added to all chambers and readings were taken with a spectrophotometer at wavelengths $560 \mathrm{Ex} / 590 \mathrm{Em}$. To determine cytotoxicity the following equation was used [(Experimental group fluorescence - Background fluorescence from media group) / (Control cell group fluorescence - Background fluorescence from media group)] x 100. 


\section{Double Labeling to Image Redistribution of Endogenous hnRNP A1}

2.5 million SK-N-SH cells were seeded into 35mm MatTek dishes with a glass coverslip bottom. Normal rabbit IgG and rabbit produced anti-hnRNP A1 antibodies were added into DMEM/F12 media at a concentration of $4 \mu \mathrm{g} / \mathrm{mL} .24$ hours after antibody addition, cells were fixed for 15 minutes with 4\% paraformaldehyde. Cells were then blocked and permeablized for 30 minutes with $6 \%$ Milk-PBS $+0.4 \%$ Triton. Cells were then washed 4 x $5 \mathrm{~min}$ with PBS. Mouse monoclonal hnRNP A1 antibodies specific to the M9 epitope of hnRNP A1 were added at a dilution factor of 1:1000 for 1 hour at room temperature in PBS to label endogenous hnRNP A1. Cells were washed $4 \mathrm{x}$ 5min with PBS. Anti-mouse Fluorescein labeled secondary antibody was added at 1:1000 for 50 min at RT in PBS. Cells were then washed and imaged in PBS.

Quantification of endogenous hnRNP A1 redistribution was performed by comparing the densitometric mean of the FITC fluorescence expression of the nucleus vs. the cytoplasm of 10 cells per group using AxioVision Software.

\section{Statistics}

Statistical analyses were performed in GraphPad Prism software using either student t-test or one-or two-way ANOVA. Significant differences depicted as standard error measurement $*<0.05, * *<0.01, * * *<0.001$, ns $=$ not significant.

\section{Aim 2 Methods}

\section{Cells}

SK-N-SH cell line (ATCC, HTB-11), an immortalized human neuroblastoma cell line was maintained in Dulbecco's modified Eagle's medium/F12 supplemented with $10 \%$ fetal bovine serum and $1 \%$ penicillin-streptomycin antibiotics. $100 \mathrm{~mm}$ plates were seeded for the RNA immunoprecipitation, Real Time PCR analysis, and Western blotting. $10^{5} \mathrm{SK}-\mathrm{N}-\mathrm{SH}$ cells were seeded per well into $500 \mathrm{uL}$ of complete DMEM/F12 media into 8 well chamber slides (Corning \#354632) for RNA granule immunocytochemistry experiments.

\section{Exposure of SK-N-SH Cells to Antibodies in vitro}

Anti-hnRNP A1 antibodies were obtained from Abcam (ab4791-rabbit polyclonal, ab5832 - mouse monoclonal) and Millipore (05-1521- mouse monoclonal; 04-1469- mouse monoclonal). The anti-hnRNP A1 antibodies are specific for hnRNP A1-M9 and have been shown to overlap the M9 immunodominant epitope recognized by IgG isolated from MS patients ${ }^{12}$. Isotype-matched anti-rabbit IgGs were obtained from 
Abcam (ab107866) and Millipore (12-370). Antibodies were added to the culture media of SK-N-SH cells at a concentration of $8 \mathrm{ug} / \mathrm{ml}$ for each assay as determined in previous studies ${ }^{1}$. Primary antibodies utilized for protein detection by Western blot were as follows: spastin (SPG4-Abcam ab38150), paraplegin (SPG7-Abcam ab154989), spartin (SPG20-Abcam ab94950), and hnRNP A1-(Millipore 05-1521). Antibodies were used at manufacturers recommended concentration. Antibodies utilized for immunochemistry were as follows: TAR-DNA Binding Protein (TDP-43 (Millipore MABN45)), GW-182 (Abcam ab70522), or hnRNP A2/B1 (Abcam ab6102).

\section{RNA Immunoprecipitation}

SK-N-SH cells were cultured in $100 \mathrm{~mm}$ cell culture dishes. Cells were scraped and lysate extracted. Lysate was added to protein $\mathrm{A} / \mathrm{G}$ beads labeled with either antihnRNP A1 antibody (ab4791) or anti-IgG (Millipore 12-370) as a control for non-specific binding. Samples were rotated overnight at $4^{0} \mathrm{C}$. The following day, bound proteins were eluted from the protein $\mathrm{A} / \mathrm{G}$ beads. Eluent from both samples as well as control SK-N-SH lysate was run on SDS gels and probed for hnRNP A1 to confirm specific binding of hnRNP A1 protein. RNA was then extracted from the eluents. RNA concentration and quality was determined by $260 / 230$ ratio by Nanodrop (ThermoFisher Nanodrop 1000). RNA was used as a template for first strand synthesis to make cDNA by a High Capacity cDNA Reverse Transcription kit (Applied Biosystems 4368814) using a thermocycler. cDNA concentration and quality was determined by Nanodrop. cDNA was used to perform quantitative real-time PCR probing for potential RNA binding partners to hnRNP A1 protein.

\section{Determination of Protein Levels of Antibody Treated SK-N-SH Cells by Western Blot}

SK-N-SH cells seeded in $100 \mathrm{~mm}$ dishes were treated with either anti-hnRNP A1 antibodies, anti-IgG antibodies, or were left untreated. Antibody treatments were at a concentration of $8 \mathrm{ug} / \mathrm{ml}$ in DMEM/F 12 complete media for 48 hours. Following antibody treatment, cells were scraped and lysates were centrifuged 5 min @ 2500 rpm. Supernatants were heated with 2x running buffer for 5 minutes @ 95C and then samples were loaded onto $10 \%$ sodium dodecyl sulfate polyacrylamide gel electrophoresis (SDSPAGE) and run at $100 \mathrm{~V}$ for 1.5 hours. Gels were then transferred onto nitrocellulose membranes at $100 \mathrm{~V}$ for 1.5 hours. Membranes were blocked with $6 \%$ milk-TBS for 40 minutes at room temperature. Primary antibodies were then added overnight at $4 \mathrm{C}$ at manufacturer's suggested concentration. Primary antibodies were as follows: SPG4 Abcam ab38150, SPG7 - Abcam ab154989, SPG20 - Abcam ab94950, and hnRNP A1 (Millipore 05-1521). The next day, membranes were washed 4 x 5 min with TBS. Secondary anti-HRP antibodies were then added for 50 minutes at room temperature at 1:10000 dilutions. Membranes were washed 4x5 minutes with TBS-Tween. Membranes were then developed and imaged. 


\section{Immunocytochemistry of hnRNP A1-M9 Antibody Colocalization with Neuronal Granules}

$10^{5} \mathrm{SK}-\mathrm{N}-\mathrm{SH}$ cells were seeded per well into 500uL of complete DMEM/F12 media into 8 well chamber slides (Corning \#354632). Anti-hnRNP A1 and control IgG antibodies were labeled with Alexafluor 488 and added to the culture media of SK-N-SH cells at a concentration of $8 \mathrm{ug} / \mathrm{ml}$ for each assay as determined in previous studies ${ }^{1}$. 48 hours following antibody addition, cells were fixed with 4\% paraformaldehyde for 30 minutes at room temperature. Fixed cells were then blocked and permeabilized with $6 \%$ Milk-PBS $+0.4 \%$ TritonX-100. Cells were then labeled for TDP-43 (stress granules), GW-182 (P bodies), or hnRNP A2/B1 (transport granules) at the manufacturer's suggested concentration overnight at $4 \mathrm{C}$. Following primary antibody incubation, cells were washed 4 x 5 minutes with PBS. Cells were treated with secondary anti-mouse Cy3 at manufacturer's suggested concentration for 2 hours at room temperature. Cells were washed 4 x 5 minutes with PBS, mounted with DAPI mounting medium, and imaged. Colocalization events were quantified based on average number of colocalizations. Colocalization events were counted and compared to number of cells in each image resulting in a ratio of colocalization events to total number of cells. Approximately 500 cells were counted per group. Data was analyzed by one-tail t-test based on previously observed trend ${ }^{1}$. (P values $\left.* \leq 0.05 ; * * \leq 0.01 ; * * * \leq 0.001\right)$.

\section{Aim 3 Methods}

\section{Animals}

C57BL/6J female mice were purchased from Jackson Laboratories (Bar Harbor, ME). Mice were 11 weeks of age at experimental onset and were housed on a 12:12 hour light:dark cycle, with standard chow and water freely available. All animal procedures were reviewed and approved by the University of Tennessee and Veterans Affairs Medical Center - Memphis Institutional Animal Care and Use Committees.

\section{Induction of Experimental Autoimmune Encephalomyelitis/Antibody Injections}

On day 0 , mice were immunized with $150 \mathrm{ng}$ of pertussis toxin via intraperitoneal (ip.) injection and $200 \mathrm{ul}$ of myelin oligodendrocyte glycoprotein (MOG) $35-55 /$ complete Freund's adjuvant (CFA) emulsion subcutaneously (100 ul between the shoulders and hips). The following day (day 1), each mouse was injected with an additional $150 \mathrm{ng}$ of pertussis toxin. Pertussis toxin and $\mathrm{MOG}_{35-55} / \mathrm{CFA}$ were obtained from Hooke Laboratories (cat no. EK-2110) (Lawrence, MA). Mice were checked daily for clinical signs of EAE (see clinical scoring section below). Upon first clinical signs, mice were injected ip. with either phosphate buffered saline (PBS), mouse IgG2b (isotype control) (Millipore MABC006), or mouse anti-hnRNP A1-M9 (Millipore 04-1469), whose 
epitope overlaps with the immunodominant epitope of IgG isolated from MS patients. Each animal received three injections of $100 \mathrm{ug}$ of antibody per injection every other day from the initial signs of EAE.

\section{Clinical Scoring}

Clinical scoring was adapted from "Appendix A: Mouse EAE Scoring Guide" found in the protocol of the Active Induction EAE Kit from Hooke Laboratories (Hooke cat no. EK-2110). The scoring system is composed of scoring values ranging from 0-5.0 increasing at increments of 0.5 . A score of 1.0 serves as the initial sign of disease onset and is scored as an observation of a 'limp tail', whereas 5.0 is moribund. Mice with scores of 4.0 or higher for more than two days are euthanized and given a score of 5.0 for the remainder of the study. Mice were scored daily throughout the study. Additionally, a notation of whether "spastic gait" was observed in an individual animal was recorded. "Spastic gait" was described as abnormally "stiff" or "flexed" extremities, compared to 'typical' EAE, when mice develop a "limp" paralysis in which their legs drag.

\section{Analyses of Neurodegeneration by Fluoro-Jade C Staining of Degenerating Neural Elements}

Mice were either euthanized by perfusion after two consecutive days of a 4.0 or higher score or at the end of the study. Intracardiac perfusion was performed via the aorta. Mice were perfused with $50 \mathrm{ml}$ of ice cold PBS until fluid ran clear, followed by $250 \mathrm{ml}$ of ice cold 4\% buffered paraformaldehyde. Brain and spinal cords were extracted and post-fixed in 4\% paraformaldehyde for 48 hours at $4 \mathrm{C}$. Brain tissues were sectioned at 30 um utilizing a sliding microtome. Spinal cord tissue was paraffin embedded and sectioned at $10 \mathrm{um}$. Sections were stained with hematoxylin and eosin and were used as reference. Fluoro-jade C (Millipore AG325) was utilized to label degenerating neural elements in both fixed, frozen brain tissue as well as paraffin embedded, fixed, spinal cord tissue. Fluoro-jade $\mathrm{C}$ is a fluorescent derivative that stains degenerating neurons selectively ${ }^{147}$. The Fluoro-jade C protocol was adapted from Kanthasamy, AG et. al ${ }^{148}$. Brain and spinal cord sections labeled with Fluoro-jade $\mathrm{C}$ were analyzed while referencing the Interactive Atlas Viewer (atlas.brain-map.org) and Brown et al ${ }^{148}$, which contained a comprehensive Fluoro-Jade C staining map of the entire CNS in MOG induced $\mathrm{EAE}_{35-55}$. Quantification of Fluoro-jade $\mathrm{C}$ intensity was performed using image $\mathrm{J}$ software with a method adapted from Burgess et al ${ }^{149}$. A comprehensive analysis was performed throughout the brain and spinal cord to determine specific regions which had increased neurodegeneration. Specific regions of the brain were imaged in order to determine if neurodegeneration was statistically different between groups based upon the mean fluorescence intensity from representative images of the specific region. The mean fluorescence intensity of 30 total images from 3 animals per group for Arbor Vitae and Corpus Callosum sections and 20 total images from 3 animals for VSCT sections were used in each statistical analysis. 


\section{CHAPTER 3. ANTI-HNRNP A1 ANTIBODIES PENETRATE AND CAUSE DELETERIOUS EFFECTS IN NEURONS IN VITRO}

\section{Introduction}

Antibodies have been implicated in the pathogenesis of a number of autoimmune diseases such as rheumatoid arthritis (RA), systemic lupus erythematosus (SLE), paraneoplastic syndromes, multiple sclerosis (MS), and human T-lymphotropic virus type 1 (HTLV-1) associated myelopathy/tropical spastic paraparesis (HAM/TSP) (a viral model of MS). ${ }^{93,94,97,98,150-154}$ The dominant theory asserts that upon cellular injury intracellular antigens are exposed to the adaptive immune response allowing for antibody response to the target antigen. It is generally believed that transected axons are vulnerable to an autoimmune response while healthy neurons are considered protected from a direct pathogenic effect. The method by which antibodies cause disease is an ongoing area of research, however current data proposes that interactions between antibodies and various intracellular molecules cause inflammation, altered cellular messaging, and apoptosis. ${ }^{155}$ The antibody response to hnRNPs seen in SLE patients provides some insight into this process of antibody-mediated disease. SLE patients produce antibodies to hnRNP P2. ${ }^{156}$ Following apoptosis of target cells, hnRNP P2 is transported to the cell surface and therefore is available to generate an autoimmune response. ${ }^{156}$ In neurological disease, previous data suggested that only neuronal surface antigens were reliable targets for pathogenic autoimmune responses. ${ }^{157,158}$ This concept is now being challenged. Current data suggest that antibodies have the ability to enter neurons in an epitope specific manner. In autoimmune retinopathy, autoantibodies to enolase, a cytoplasmic glycolytic enzyme, were shown to penetrate neurons and alter the function of enolase. ${ }^{159}$ Another example is present in a model of stiff man syndrome in which anti-amphiphysin antibodies entered neurons and co-localized with presynaptic markers and altered gamma-aminobutyric acid (GABA) release in vivo. ${ }^{160}$ Such studies address important issues. However, to better understand the contribution of immune responses to intraneuronal antigens in the pathogenesis of immune-mediated neurological diseases, more information is required.

MS is a neurodegenerative disorder resulting in demyelination of neurons, which is increasingly associated with grey matter and neuronal degeneration. MS and HAM/TSP patients have been shown to produce autoantibodies to the intracellular RNA binding protein heterogeneous nuclear ribonucleoprotein A1 (hnRNP A1). ${ }^{93,94,96-98,154}$ IgG from MS and HAM/TSP patients reacted specifically with an epitope within the M9 region of hnRNP A1, its nuclear localization/export sequence (NLS/NES). These antibodies decreased neuronal firing and contributed to the death of neuronal cultures. ${ }^{94,154}$ However, until now it remained unknown if anti-hnRNP A1 antibodies have the ability to penetrate neuronal cells. Because autoantibodies to the M9 region of hnRNP A1 appear unique to MS and HAM/TSP patients and are not seen in healthy individuals, we decided to investigate whether antibodies that target the hnRNP A1-M9 epitope can penetrate healthy neuronal cells and examine the possible deleterious effects that they might induce. 


\section{Direct Antibody Addition into Growth Media Results in Antibody Penetration into Neuronal Cells}

In order to investigate the role of antibodies to hnRNP A1 on neuronal cells, we determined whether the antibodies had the ability to penetrate SK-N-SH neuronal cells. We utilized commercially available anti-hnRNP A1 antibodies that recognize the M9 region of hnRNP A1 and control IgG antibodies to determine the antibodies' ability to penetrate neuronal cells. To visualize antibody penetration, we labeled the antibodies with a red fluorescent dye - Atto-550-NHS dye as previously described. ${ }^{161}$ Atto-550NHS is a new label with high molecular absorption and quantum yield. Excitation and emission for Atto-550-NHS (Ex.556/Em.578nm) is similar to a well-known Cy3 dye. Atto-550-NHS labeled antibodies were added directly to the cell growth media at different concentrations $(2,4,6$, and $8 \mu \mathrm{g} / \mathrm{mL})$ without any additional reagents. Cells were examined under Zeiss AxioObserver1 microscope in phase and fluorescence modes 24 and 72 hours following treatment. Cells treated with fluorescent label itself did not uptake the dye, suggesting that Atto-550-NHS was not responsible for antibody penetration (data not shown). At the 24-hour point, the anti-hnRNP A1 and control IgG antibodies were found inside neuronal cells (Figure 3-1A). Additionally, at 72 hours, penetration was seen in both groups. Interestingly, quantification of the percentage of cells penetrated by antibodies reveals that the anti-hnRNP A1 antibodies penetrate the neuronal cells more readily than control $\operatorname{IgG}$ (Figure 3-1B). These findings suggest that antibodies possess the ability to penetrate neuronal cells and that the anti-hnRNP A1 antibodies penetrated more readily than control antibodies.

\section{Antibodies to hnRNP A1 Penetrate Neuronal Cells and Co-localize with Early Endosomes}

Next, we sought to examine the mechanisms of cellular penetration. Since endocytosis is involved in the uptake of numerous molecules from the extracellular environment, we set out to determine whether endosomes were involved in antibody entry into neurons. To accomplish this task, we performed localization studies and tested whether antibodies co-localized with early endosomes. Early endosomes were prelabeled with GFP BacMam reagent (green) and antibodies with Atto-550-NHS (red). As early as 6 hours after antibody addition, anti-hnRNP A1 antibodies co-localized with early endosomes (Figure 3-2A). Maximum co-localization was seen at 24 hours after antibody addition (Figures 3-2A and 3-2B). At 48 hours, antibody co-localization with early endosomes was still present (Figure 3-2A), however the number of endosomes that contained antibodies was significantly less than at 24 hours. The fact that co-localization is seen at multiple time points rather than just a single window of time, suggests that antibodies continually penetrate neuronal cells as long as there are still free antibodies in the extracellular media. These results indicate that endocytosis appears to be the mechanism by which antibodies penetrate neuronal cells. 

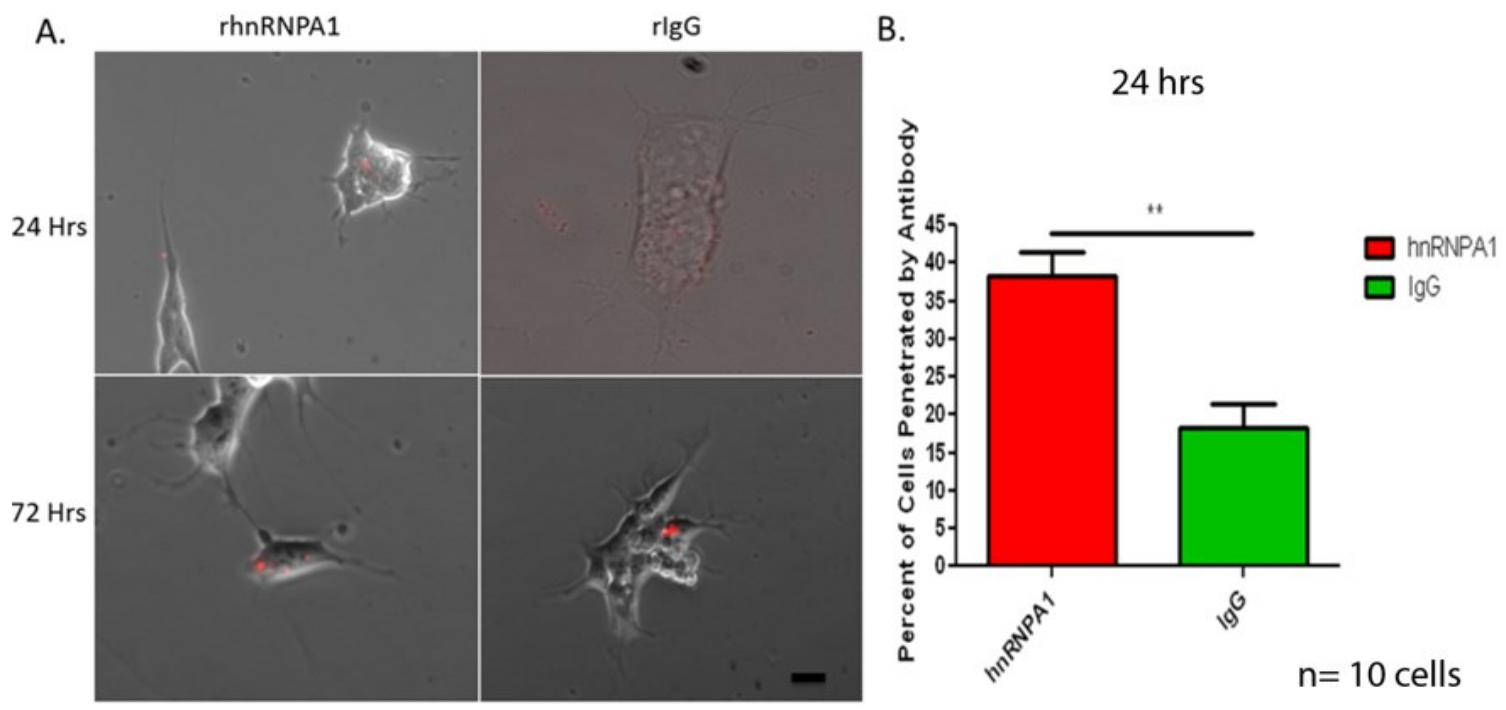

Figure 3-1. Localization of antibodies by immunocytochemistry in SK-N-SH cells. A. Following addition of antibodies to the media, Atto-550-NHS labeled anti-hnRNPA1 antibodies specific to the M9 epitope and control antibodies (both in red) were found to penetrate neuronal cells. B. Quantification of antibody penetration percentage between the anti-hnRNP A1 treated and control rabbit IgG treated cells was performed using AxioVision Software. Five images were used per treatment from the 24 hour images. Antibody penetrated cells were divided by the total number of cells to give a percentage of antibody penetration. (Cells with antibodies per image/Total cells per image) x $100=$ Penetration Percentage. Data was analyzed with one way ANOVA with standard error measurement (SEM). $* \mathrm{P}<0.05, * * \mathrm{P}<0.01$. 
A.

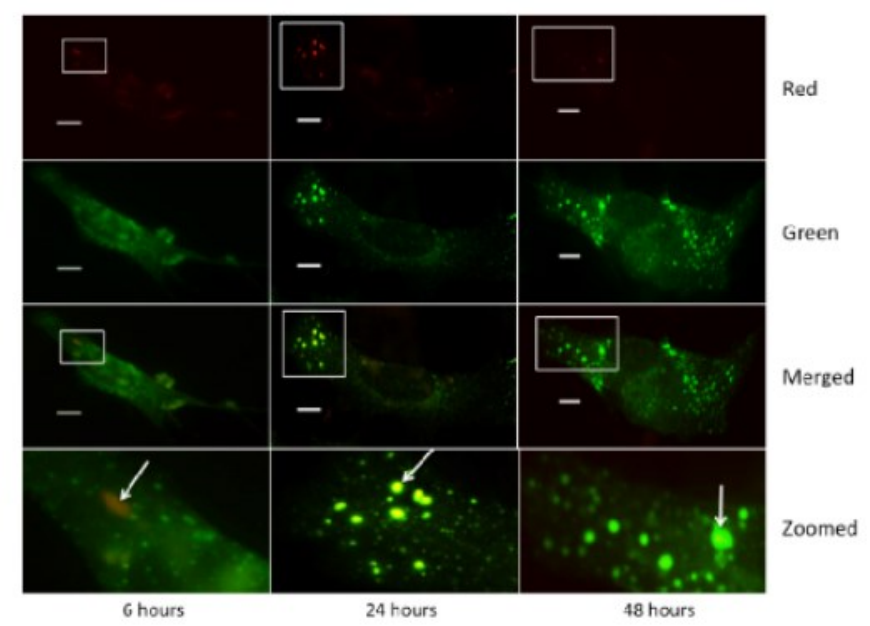

B.

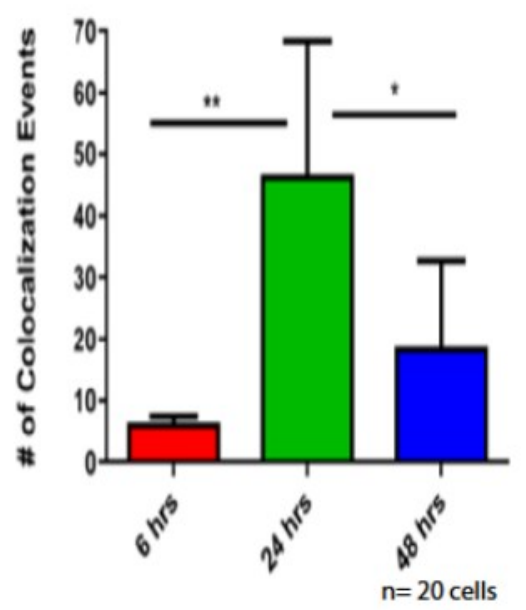

Figure 3-2. Antibodies co-localize with early endosomes.

Early endosomes labeled with BacMam particles GFP (green), antibodies with Atto550NHS (red). Examples of co-localization (yellow) are shown in boxes. Arrows in highpowered images point to colocalized antibody example. Scale bar $=10 \mu \mathrm{m}$. 2B.

Quantification of antibodies co-localized to early endosomes at 6, 24 and 48 hours after antibody addition to the media. Images were taken on fixed cells and number of colocalized antibodies was calculated per cell at a given time point. Events were calculated in 10 cells per time point from two experiments ( 20 cells total per time point). Groups were analyzed with one-way ANOVA with Newman-Keuls multiple comparison test using SEM. $* \mathrm{P}<0.05, * * \mathrm{P}<0.01$. 


\section{Antibodies to hnRNP A1 and IgG Penetrate Neuronal Cells via Clathrin Mediated Endocytosis and Not via Caveolar Mediated Endocytosis}

Two main types of endocytosis occur in neuronal cells, clathrin and caveolar mediated endocytosis. ${ }^{162,163}$ Molecules endocytosed into cells can traffic through one or both pathways. We examined whether neuronal cells uptake antibodies via one or both of these pathways. To answer this question, we used competitive inhibitors of the individual pathways to determine whether antibody penetration was blocked. To evaluate clathrin-mediated endocytosis we used Pitstop2 reagent, which selectively inhibits clathrin via inhibition of the clathrin terminal domain. ${ }^{163,164}$. With clathrin-mediated endocytosis inhibited, we witnessed complete blockage of the penetration of the antibodies, thus illuminating clathrin-mediated endocytosis is at least one mechanism by which antibodies penetrate neuronal cells (Figure 3-3A). To assess whether caveolarmediated endocytosis was also involved in antibody entry into the cell we used nystatin, a lipid raft inhibiting agent, to block caveolar-mediated endocytosis ${ }^{165}$. When caveolarmediated endocytosis was blocked, we observed a standard amount of antibodies inside neuronal cells (Figure 3-3A). Cells with nystatin did not differ from cells without any blocking reagent. This suggests that caveolar-mediated endocytosis is not involved in the intracellular penetration of antibodies (Figure 3-3A). To confirm our observation biochemically, we performed co-immunoprecipitation experiments. In these experiments, anti-hnRNP A1 antibodies or control rabbit IgG were bound to agarose-A beads. The whole cell lysate was incubated with the beads overnight, complexes were eluted, immobilized onto PVDF membranes and probed with anti-clathrin antibodies. Beads without antibodies (B) served as negative control (Figure 3-3B). A clathrin band is seen in the whole cell lysate lane (L) confirming its presence in SK-N-SH cells. Signal is enriched in both the control IgG and anti-hnRNP A1-M9 lanes, but not in the blank lane (B), indicative of antibody binding to clathrin, and thus confirming the specificity of observation seen by immunocytochemistry. Taken together, these results demonstrate that antibodies penetrate neuronal cells via clathrin-mediated endocytosis.

\section{Anti-hnRNP A1 Antibodies Deplete Cellular ATP}

Based upon our previous data showing antibody entry into neurons, we hypothesized that there would be altered cellular events related to the presence of antibodies inside the cells. Previously, we showed that transfection of anti-hnRNP A1M9 antibodies into dNT-2 neurons resulted in expression of neurodegenerative markers and cell death within 7 days. ${ }^{154}$ Transfection of normal rabbit IgG did not have the same effect. Thus, it appears that anti-hnRNP A1-M9 antibodies play a role in neuronal degeneration. In this study, we carried out all experiments without transfection and examined the possible effects of these antibodies on SK-N-SH neuronal cells. Since ATP is essential to cell health and function, we determined if cellular ATP levels, a measure of cellular viability, were altered by anti-hnRNP A1 antibodies. We performed the CellTiter-Glo Luminescent Cell Viability Assay to determine the ATP levels of the neuronal cells in untouched as well as in the precence of the antibodies. In these experiments we used unlabeled anti-hnRNP A1 or IgG. Our results reveal that ATP levels 

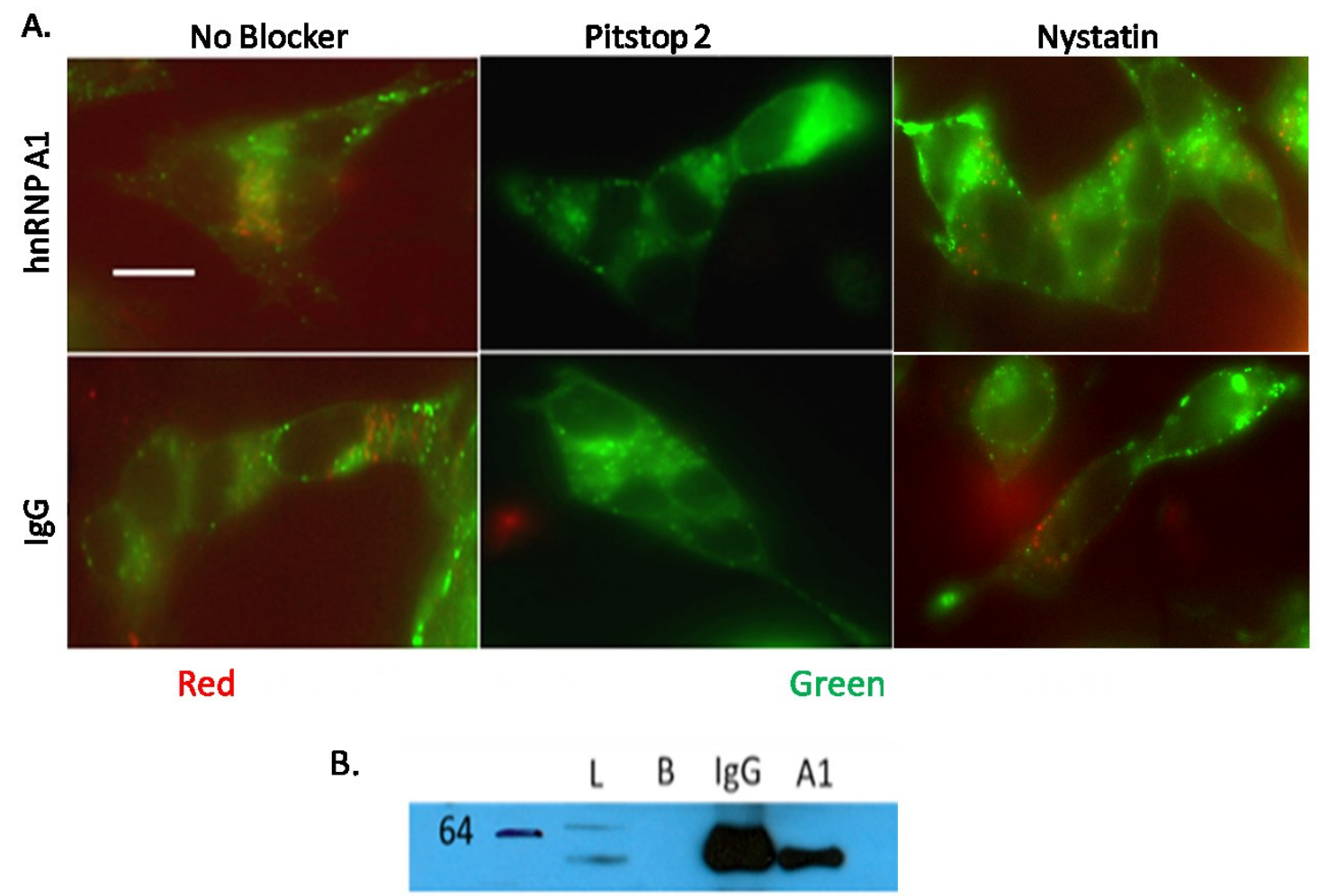

Figure 3-3. Antibodies (labeled red) penetrate SK-N-SH cells via clathrinmediated endocytosis.

A. Addition of Pitstop2 reagent prevented clathrin-mediated endocytosis of both antihnRNP A1 and IgG into SK-N-SH cells. Addition of nystatin, used to block caveolar mediated endocytosis, did not block penetration. B. Clathrin immunoprecipitation on agarose A-linked anti-hnRNPA1 and control IgG. L= SK-N-SH cell lysate. $\mathrm{B}=$ Agarose A beads, $\mathrm{IgG}=$ control IgG attached to agarose $\mathrm{A}$ beads, $\mathrm{A} 1=$ anti-hnRNPA1 antibodies attached to agarose A beads. Both control IgG and anti-hnRNP A1 antibodies bound clathrin, confirming the observation by immunocytochemistry. 
were compromised by as early as 48 hours after anti-hnRNP A1 antibody addition (Figure 3-4). Addition of normal rabbit IgG did not significantly affect ATP levels in these cells. The alteration of cellular ATP levels induced by anti-hnRNP A1 antibodies could lead to a multitude of deleterious downstream effects on the neuronal cells.

\section{Anti-hnRNP A1 Antibodies Are Not Cytotoxic}

Considering that anti-hnRNP A1-M9 antibodies diminished the viability of neuronal cells, we performed the Cytotox-ONE Cytotoxicity Assay to determine the possible cytotoxic effects of anti-hnRNP A1-M9 antibodies on neuronal cells.

Cytotoxicity in these experiments was determined by the amount of membrane damage to the cells as measured by the Cytotox-ONE Cytotoxicity Assay. SK-N-SH cells were seeded in 96-well plate in triplicate for each group: 1) untouched, 2) cells with control rabbit IgG, 3) cells with anti-hnRNP A1 antibodies. All groups were normalized based on relative fluorescence of untouched cells. Plates were evaluated at 24, 48, and 72 hours after antibody addition. There were no statistical differences in cytotoxicity between the anti-hnRNP A1 and control antibodies, suggesting that neither control rabbit IgG or antihnRNP A1-M9 antibodies were cytotoxic to SK-N-SH cells. (Figure 3-4B).

\section{Anti-hnRNP A1 Antibody Increased Apoptosis in Neuronal Cells}

Considering the effects of anti-hnRNP A1 on neuronal viability (ATP levels), we hypothesized that the anti-hnRNP A1 treated cells would show an increase in apoptosis compared to control antibodies. Caspases 3 and 7 are the executioner apoptosis caspases. In order to examine the levels of these caspases we used the ApoOne Homogenous Caspase $3 / 7$ Kit (Promega). Cells treated with anti-hnRNP A1 antibodies were compared to control IgG and untouched cells at 24, 48 and 72 hours after antibody addition. No differences were detected between the groups at $24 \mathrm{~h}$. However at $48 \mathrm{~h}$, cells treated with anti-hnRNP A1-M9 antibodies showed increased Caspase 3/7 levels in comparison to control IgG. This trend continued at the 72-hour time point showing a significant increase in Caspase 3/7 activity in the anti-hnRNP A1-M9 antibody treated group compared to controls (Figure 3-4C). These results reveal that one mechanism by which anti-hnRNP A1-M9 antibodies alter cell viability in neuronal cells is due to an increase in Caspase $3 / 7$ activity leading to apoptosis of neuronal cells.

\section{Anti-hnRNP A1 Antibodies Cause Redistribution of Endogenous hnRNP A1 Protein}

Based upon the specificity of an antibody-antigen interaction, we hypothesized that the anti-hnRNP A1 antibodies not only penetrated neurons and localized to early endosomes, but also targeted endogenous hnRNP A1 protein. In order to resolve this question, we performed double labeling experiments in which we added rabbit Atto550 labeled anti-hnRNP A1 and control antibodies to neuronal cells, fixed and permeabilized the cells and then labeled endogenous hnRNP A1 with mouse monoclonal anti-hnRNP 
Figure 3-4. Anti-hnRNP A1 antibodies caused an increase in apoptosis and depletion of ATP levels.

A. Cellular ATP levels are significantly reduced when SK-N-SH cells were exposed to anti-hnRNP A1 antibodies. B. Antibodies were not cytotoxic to the SK-N-SH cells

(Percent cytotoxicity was normalized based on the numbers detected in untouched cells). C. In contrast to control antibodies, reduced levels of caspase 3/7 were seen at 48 and 72 hours after anti-hnNRP A1 antibodies were added to the media. Results were analyzed with two-way AN 
A.

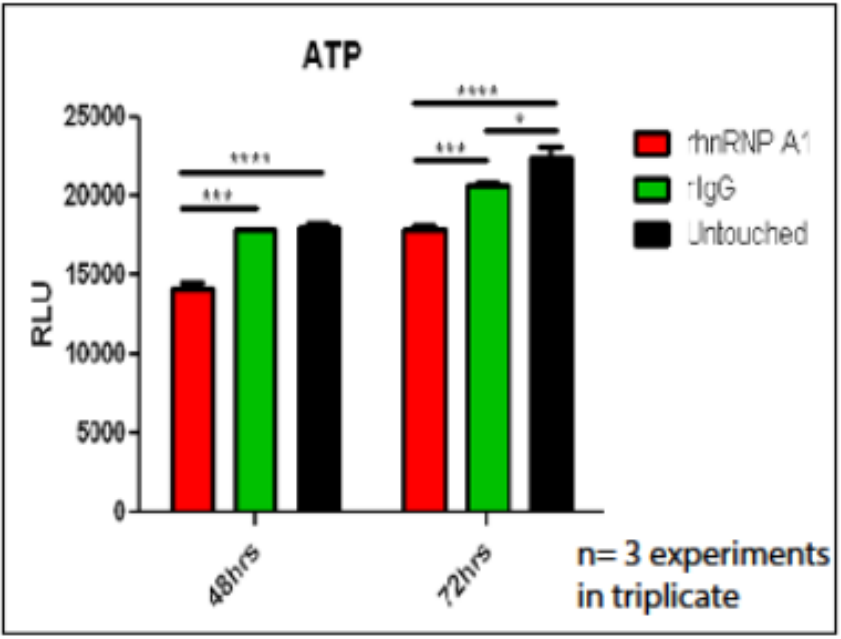

B.

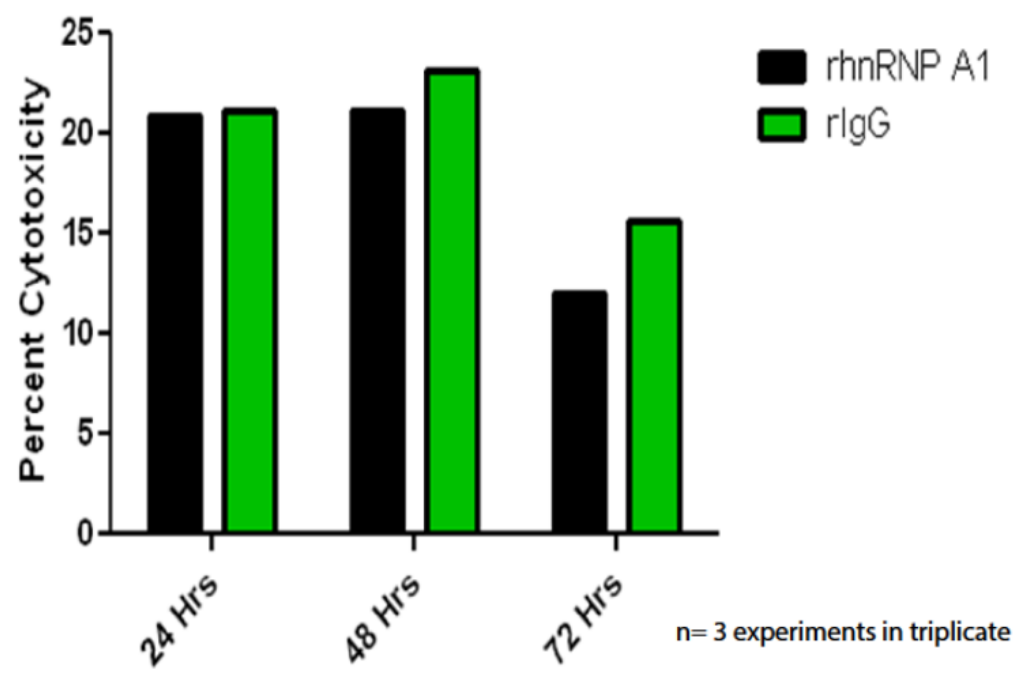

C.

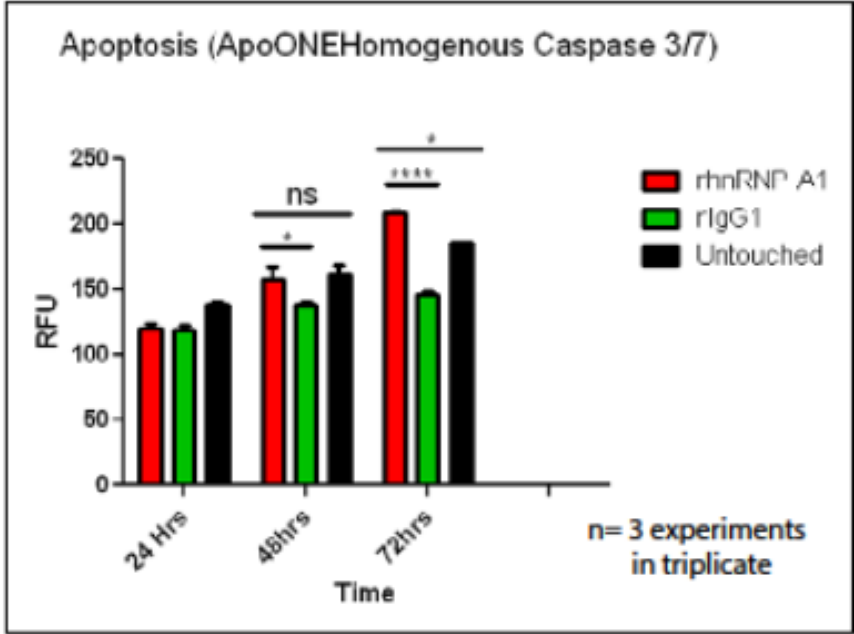


A1 followed by secondary anti-mouse FITC antibodies (Figure 3-5). In contrast to control antibodies, our results reveal that following anti-hnRNP A1-M9 antibody addition there is a redistribution of endogenous hnRNP A1 from being primarily nuclear to being equally distributed in the nucleus and in cytoplasm (Figures 3-5A and 3-5B). Total densitometric means of FITC expression were quantified for both the nuclear and cytoplasmic portions of the cells in all experimental groups using Axiovision software. The densitometric mean of FITC expression of the nuclear and cytoplasmic cellular portions was used to quantify a nuclear to cytoplasmic ratio for each experimental group (Figure 3-5B). These analyses indicate that the anti-hnRNP A1 treated cells have a significantly altered nuclear to cytoplasmic expression ratio of endogenous hnRNP A1 when compared to untouched control and the IgG treated cells. Based upon the data presented, it suggests that anti-hnRNP A1 antibodies penetrate neuronal cells and have a specific effect upon endogenous hnRNP A1 protein resulting in the protein getting trapped in the cytoplasm. Alteration of the endogenous protein could have downstream effects upon mRNA binding partners transported by hnRNP A1 under normal conditions.

\section{Conclusions}

The role of autoantibodies and their ability to target intracellular antigens is an important focal area of research for autoimmune disorders. The notion that cellular injury is initiated and swiftly followed by intracellular antigens being exposed to the adaptive immune response resulting in the ability of antibody response to the target antigen prevails as the dominant theory. Autoantibodies produced by patients with autoimmune disorders have been implicated in the pathogenesis of various diseases such as SLE, RA, paraneoplastic syndromes, MS and HAM/TSP. ${ }^{12,93,94,97,98}$ In previous work it has been shown that patients with MS and HAM/TSP produce autoantibodies to the nuclear shuttling protein hnRNP A1, and specifically to its M9 region. ${ }^{12}$ The autoantibodies specific to hnRNP A1 have been implicated in the pathogenesis of MS and HAM/TSP, however until now it was not clear how these antibodies might cause target cell damage.

Anti-recoverin antibodies that are present in patients with cancer associated retinopathy (CAR) syndrome were previously shown to penetrate E1A.NR3 cells via endocytosis. ${ }^{144}$ In this study, we have shown that antibodies to hnRNP A1 are capable of penetrating SK-N-SH neuronal cells through clathrin-mediated endocytosis. Compared to control antibodies, the anti-hnRNP A1 antibodies penetrated neuronal cells more readily and showed a higher level of co-localization within early endosomes. Interestingly, anti-hnRNP A1 antibodies added directly into the media penetrated at nearly the same efficiency seen previously through transfection methods of anti-hnRNP A1 antibodies into the SK-N-SH neuronal cells. ${ }^{12}$ The fact that the method of antibody penetration occurs through endosomes gives great insight because endocytosis is a general method of cellular uptake of various molecules and it occurs in various neuronal cell types. Therefore, it would be important to note that this method of antibody penetration might be taking place in a multitude of neurons throughout the nervous system. Retrograde axonal transport through signaling endosomes has been shown as a 
A.

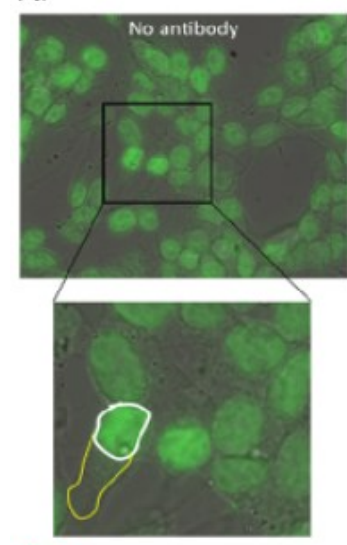

Red-labeled antibody White outline- nucleus

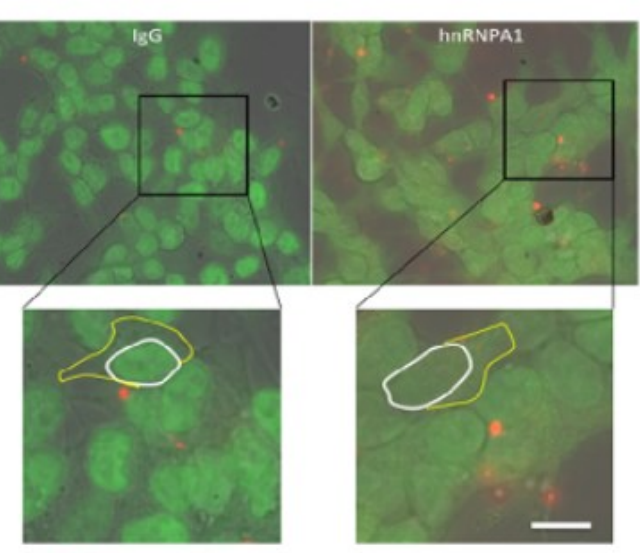

Green-endogenous hnRNP A1 protein - cytoplasm
B.

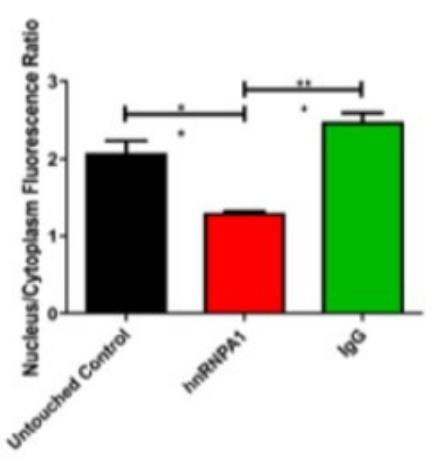

$n=20$ total cells from 3 experiments

Figure 3-5. Anti-hnRNP A1 antibodies cause redistribution of endogenous hnRNP A1 in SK-N-SH cells.

A. Atto-550-NHS labeled anti-hnRNPA1 or control antibodies (red) were added to the cells. 24 hours later cells were fixed, washed and permeabilized. Endogenous hnRNP A1 was labeled with primary mouse monoclonal antibodies followed by FITC anti-mouse secondary antibodies. White borders outline nuclei, yellow - cytoplasm. B. Quantification of endogenous hnRNP A1 redistribution in comparison to untouched cells and cells with control IgG. Nuclear to cytoplasmic fluorescence ratio calculated using densitometric means, $\mathrm{n}=20$ cells from three separate experiments. One way ANOVA was utilized using SEM. $* \mathrm{P}<0.05, * * \mathrm{P}<0.01$. 
growing area of research in neurodegenerative disorders. A link has been formulated between disruption of retrograde transport and a variety of neurodegenerative diseases 138. Various neurotrophic factors such as nerve growth factor (NGF), brain derived neurotrophic factor (BDNF), neurotrophin 3 (NT-3), and neurotrophin 4 (NT-4) have been shown to bind specific Trk receptors at axon terminals and are ultimately transported retrogradely via signaling endosomes by microtubule dependent dynein motors. ${ }^{138,166,167}$ Dynein function disruption has been shown to lead to neurodegeneration in both mice and human disease. ${ }^{138}$ Furthermore, it has also been presented that both retrograde and anterograde transport malfunction can result in a "traffic jam" of sorts, displaying that mutations in kinesins as well as dynein can influence signaling endosomes in a negative manner ${ }^{138,168}$. Of specific interest, hereditary spastic paraplegia (HSP) a disease that is clinically indistinguishable from progressive MS and HAM/TSP, exhibits the "dying back phenomena" specific to disruption of retrograde transport as the disease is seen to progress in a distal to proximal progression ${ }^{138}$. Several forms of HSP contain causative mutations of molecular motors or in other proteins that affect axonal transport indirectly. ${ }^{138,169-171}$ These studies provide insight into future studies to assess whether autoantibodies to hnRNP A1 produced by MS and HAM/TSP patients have an effect upon retrograde transport, thus providing a possible mechanism for the distal to proximal neurodegeneration seen in patients with MS.

In these studies, we show for the first time that antibodies to hnRNP A1 have the ability to penetrate healthy neuronal cells and cause negative effects on the intracellular environment. Compared to control antibodies, anti-hnRNP A1 antibodies significantly depleted cellular ATP levels and caused significant increases in caspases 3/7 levels leading to apoptosis in SK-N-SH neuronal cells. It is important to note that decrease in cellular ATP often correlates with the initiation of apoptosis leading to cell death. ${ }^{172} \mathrm{In}$ addition, we showed that anti-hnRNP A1 antibodies caused a redistribution of endogenous hnRNP A1 from being primarily nuclear to being equally nuclear and cytoplasmic. The epitope recognized by the anti-hnRNP A1 antibodies is AA 293-304, located at the C-terminal of the M9 sequence, which is hnRNP A1's NLS/NES. ${ }^{97}$ The anti-hnRNP A1 antibodies targeted this sequence and altered its nuclear-cytoplasmic transport. Importantly, Michael et al ${ }^{126}$ showed that deletion mutations in this region of M9 result in loss of nuclear import and export of hnRNP A1. Taken together, these data indicate that the anti-hnRNP A1 antibodies specifically targeted M9 and in doing so, caused a change in localization of hnRNP A1, which considering its critical role in cellular function, would likely have deleterious effects on neurons. Consequently, we hypothesize that anti-hnRNP A1 autoantibodies found in patients with MS and HAM/TSP might contribute to the pathogenicity of the disease, rather than merely disease markers.

In summary, these experiments showed that anti-hnRNP A1 antibodies penetrate SK-N-SH neuronal cells through clathrin-mediated endocytosis and enter early endosomes. In addition, the antibodies significantly altered ATP levels and increased Caspase 3/7 levels that ultimately lead to apoptosis and cell death. Further, our studies reveal that anti-hnRNP A1 antibodies also have specific effects on hnRNP A1. These 
findings have implications concerning the pathogenesis of MS and HAM/TSP, in which neuronal damage and neurodegeneration are important features. The findings presented in AIM 1 set the foundation for the next two aims. By determining the mechanism of antibody entry as well as various negative effects compromising the viability of the antibody-treated cells, the groundwork was set to delve further into a specific mechanism causing the cell death. In AIM 2 we seek to answer these questions by assessing antibodies possible role in stress granules, determining mRNA binding partners of hnRNP A1 protein, and investigating translational repression of selected genes of interest due to antibodies effect on neuronal cells. 


\title{
CHAPTER 4. ANTI-HNRNP A1 ANTIBODIES CO-LOCALIZE WITH RNA GRANULES AND ALTER SPASTIC PARAPLEGIA GENES IN NEURONS
}

\begin{abstract}
Aim 2 Introduction
Multiple sclerosis (MS) is an autoimmune disorder of the central nervous system (CNS) of unknown etiology. It is believed to occur in individuals with genetic susceptibility. ${ }^{173-176}$ Disease in these individuals might be initiated by an environmental stimulus, which results in an inflammatory response directed towards CNS targets. The resulting autoimmune response leads to demyelination and axonal degeneration within the CNS.
\end{abstract}

MS patients have been shown to produce autoantibodies to various myelin and non-myelin antigens. For example, patients develop autoantibodies to myelin oligodendrocyte glycoprotein (MOG), myelin associated glycoprotein (MAG), myelin basic protein (MBP), and proteolipid protein (PLP). ${ }^{100-103}$ These myelin autoantibodies have been found in the cerebrospinal fluid (CSF) and serum of MS patients. Contradictory studies exist as to whether these myelin-associated antibodies are pathogenic. ${ }^{11,102,104-109,177}$ In addition to myelin antigens, MS patients have been shown to produce autoantibodies to various neuronal antigens such as neurofascin (NF-186), neurofilament light chain (NF-L), and to glial antigens such as KIR4.1 and glial fibrillary acidic protein (GFAP). ${ }^{112-114,178}$ Importantly, studies have shown these target specific antibodies to be associated with increased disease progression in animal models (NF-186) and cerebrospinal fluid (CSF) from human samples (NF-L, GFAP) respectively. ${ }^{112-114}$

Autoantibodies produced by MS patients are specific to the M9 region of hnRNP A1. 1,2,11,12,95,99 Anti-hnRNP A1 antibodies enter neuronal cells through a mechanism involving clathrin-mediated endocytosis. ${ }^{1}$ Once inside neuronal cells, anti-hnRNP A1 antibodies reduce cellular ATP levels, increase apoptosis, and cause endogenous hnRNP A1 mislocalization from a primarily nuclear localization to a near 1:1 nuclear to cytoplasmic ratio. ${ }^{1}$ Furthermore, anti-hnRNP A1 antibodies transfected into human neuronal cells in vitro altered RNA levels of the spastic paraplegia genes (SPGs) spastin (SPG4), spartin (SPG20), and paraplegin (SPG7) as shown by microarray. ${ }^{12}$ The alterations in the SPGs were further confirmed in neurons purified from human brain samples of MS patients compared to normal controls by Real-Time PCR. ${ }^{12}$ Mutations within the SPGs are associated with the neurologic disease hereditary spastic paraplegia, which is clinically similar to some forms of MS. ${ }^{133}$

Three main types of granules exist within neuronal cells: stress granules (SGs), processing bodies (P bodies), and neuronal transport RNPs (also known as neuronal 'transport granules'). ${ }^{179}$ SGs develop as the result of various cellular stressors. ${ }^{179}$ SGs harbor translationally repressed RBPs and their mRNA cargo during cellular stress. Once the cell has returned to homeostasis; the SGs sort, remodel, and export the various mRNAs for reinitiation or storage. ${ }^{179} \mathrm{P}$ bodies act as sites of translational repression and degradation for the cell. ${ }^{179} \mathrm{P}$ bodies do not contain ribosomal subunits and are the waste 
management system of the cell. Neuronal transport granules act as motile granules to transport RBPs and their translationally arrested mRNA cargoes to sites of translation. ${ }^{179}$ In summary, SGs harbor translationally arrested RBPs and their cargo during times of cellular stress until homeostasis is achieved, $\mathrm{P}$ bodies manage the waste of the cell, and neuronal transport granules are responsible for transport of RBPs and their translationally arrested cargoes, which upon arrival to designated sites in the neuron, undergo translation.

In this section we sought to determine anti-hnRNP A1 antibodies interaction with the three types of neuronal granules (SGs, P bodies, and neuronal 'transport granules'). Additionally, we tested whether the SPGs (SPG4 and SPG7) are mRNA binding partners of hnRNP A1 protein. Moreover, we resolved whether anti-hnRNP A1 antibodies effect RNA expression of the mRNA binding partners. Finally, we determined downstream effects of anti-hnRNP A1 antibodies on translation of these clinically relevant mRNA.

\section{Aim 2 Results}

\section{Anti-hnRNP A1 Antibodies Colocalize with SGs but Not P Bodies or Neuronal Transport Granules}

Neuronal granules play a crucial role in the trafficking of various RBPs and their mRNA cargoes in neuronal cells. P bodies and neuronal transport granules are present when cells are in homeostasis, whereas SGs only occur during cellular stress. ${ }^{179}$ SGs harbor RBPs and their cargo in a state of translational repression during a stress response until the cell has returned to a homeostatic state. Since patients with MS produce antibodies to hnRNP A1, which is an RBP, we hypothesized that the anti-hnRNP A1 antibodies would colocalize to RBP specific granules within neurons. Previously, we have shown that anti-hnRNP A1 antibodies entered neurons by clathrin-mediated endocytosis to a higher degree than control $\operatorname{IgG}(\mathrm{p} \leq 0.01) .{ }^{1}$ Therefore, we further hypothesized that anti-hnRNP A1 antibodies would cause cellular stress resulting in SG formation at higher levels than control IgG. To answer this question, anti-hnRNP A1 (ab4791) specific antibodies as well as isotype-matched control IgG labeled with Alexafluor 488 were added to the media of SK-N-SH neuronal cells in culture. After a 48 hour incubation, cells were fixed and labeled for SGs (TDP-43), P bodies (GW182), and neuronal transport granules (hnRNP A2B1) utilizing neuronal granule components previously identified by Kiebler et al. ${ }^{179}$ Antibody treated groups were compared to untreated control SK-N-SH cells. Our results revealed that both anti-hnRNP A1 antibodies and control IgG resulted in SG formation and both colocalized with SGs (Figure 4-1A). However, anti-hnRNP A1 antibodies penetrated neuronal granules and colocalized with SGs more readily and the differences were statistically significant $(\mathrm{p} \leq 0.05)$, suggesting that hnRNP A1 antibodies specifically bound hnRNP A1 within SGs (Figure 4-1B, Table 4-1). Neither of the antibodies colocalized with P bodies or neuronal transport granules (not shown). 
Figure 4-1. Antibodies co-localize with stress granules in SK-N-SH neurons.

A. SK-N-SH cells were seeded into 8 chamber slides. Cells were treated with either antihnRNP A1-M9 antibodies, IgG, or left untouched. Following a $48 \mathrm{hr}$ incubation, cells were fixed with 4\% Paraformaldehyde, blocked and permeabilized, and stained for stress granules with anti-TDP-43 antibodies. The figure shows that both anti-hnRNP A1-M9 antibodies and control IgG cause stress granule formation and colocalize to stress granules. B. Anti-hnRNP A1-M9 antibodies colocalize at a two-fold higher percentage than IgG. The increase in colocalization was quantified using a one-tailed t-test based on a previously observed trend. $* \mathrm{P}<0.05, * * \mathrm{P}<0.01$. 


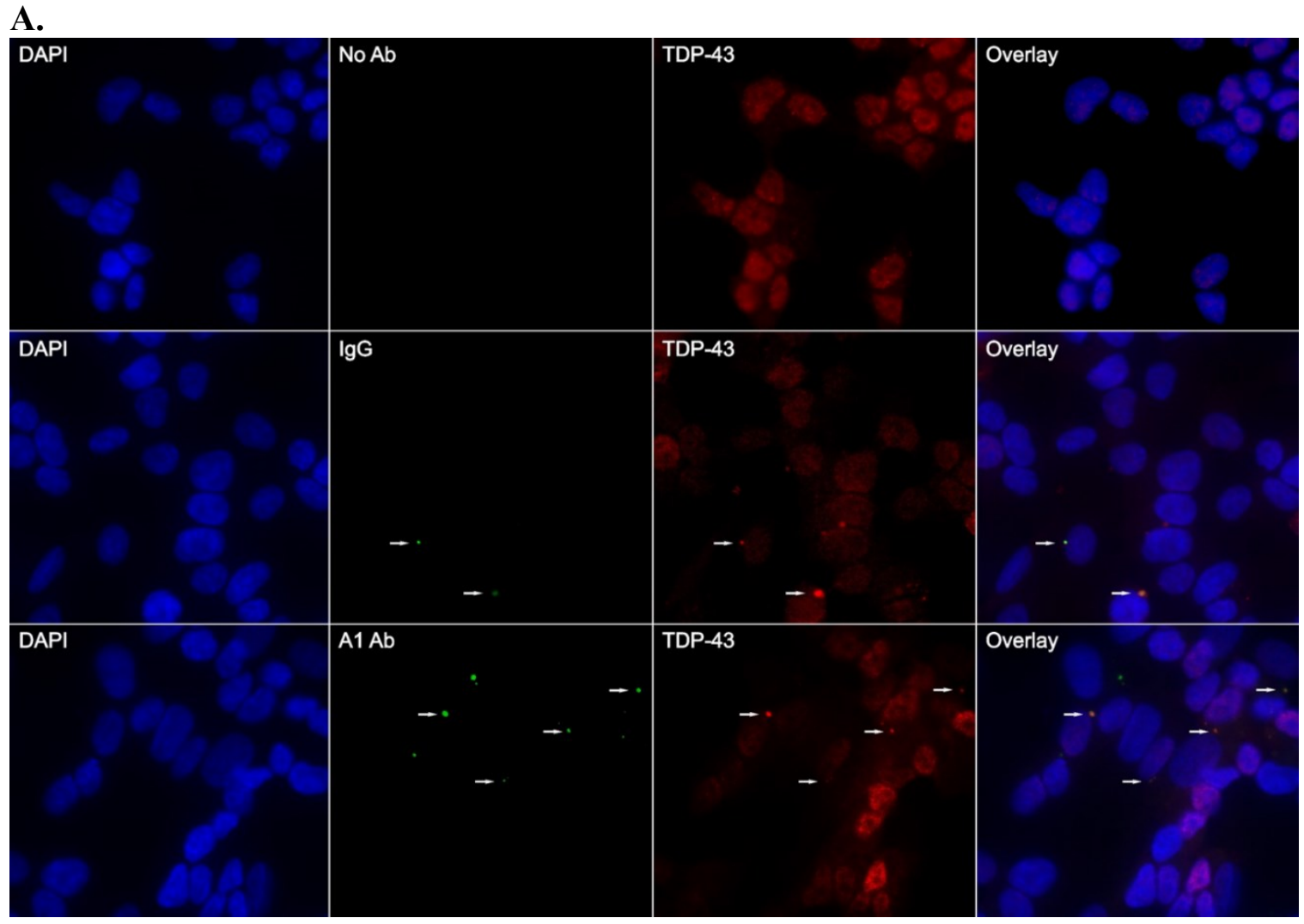

B.

\section{Antibody-SG Colocalization}

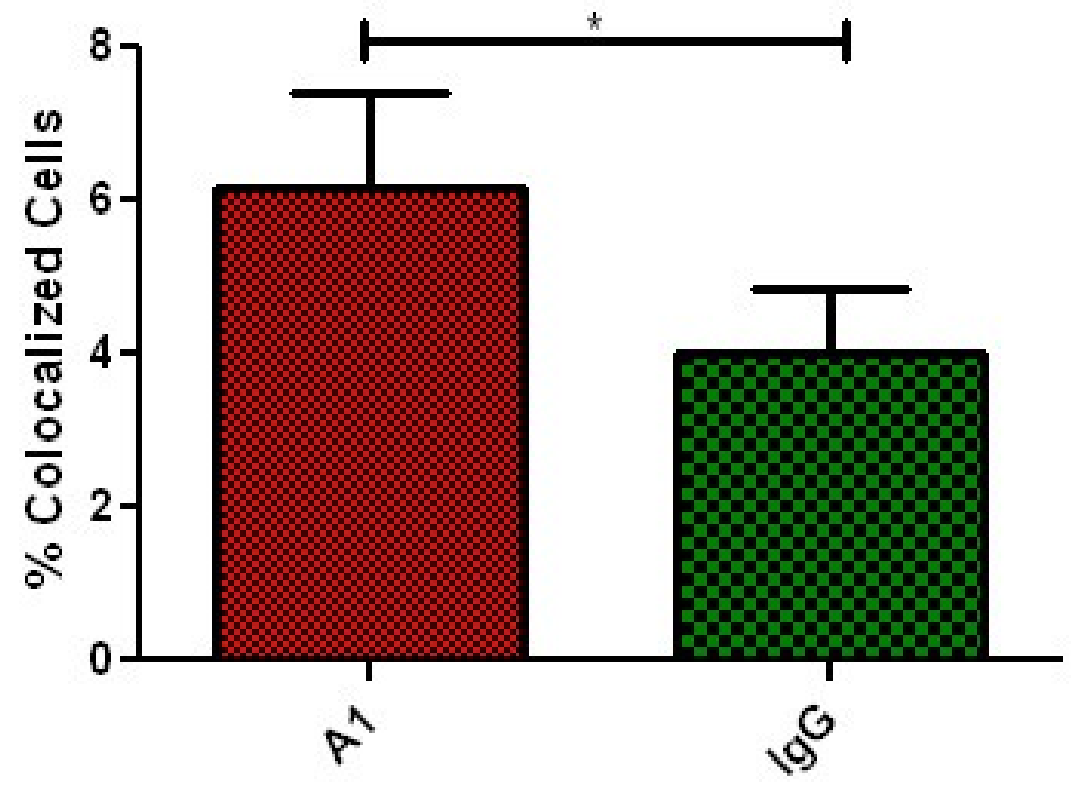


Table 4-1. Quantification of antibody colocalization with stress granules.

\begin{tabular}{lcc}
\hline Culture condition & IgG & A1 \\
\hline Cells counted & 537 & 530 \\
$\begin{array}{l}\text { Colocalizations } \\
\begin{array}{l}\text { Colocalization to cell } \\
\text { percentage }\end{array}\end{array}$ & 19 & 30 \\
\hline
\end{tabular}




\section{SPG4 and SPG7 RNA Bind hnRNP A1 Protein}

After determining that both anti-hnRNP A1 antibodies and control IgG colocalize within SGs, we sought to determine whether anti-hnRNP A1 antibodies have a specific effect on the RNA metabolism of hnRNP A1 protein's RNA cargo. In order to accomplish this goal we needed to determine the RNA binding partners of hnRNP A1 protein. Previous studies have shown that anti-hnRNP A1 antibodies transfected into neuronal cells resulted in altered levels of specific genes when determined by microarray. ${ }^{12}$ Furthermore, these studies also confirmed the alteration of a subset of these genes in neurons isolated from MS patient brain compared to control brain. ${ }^{12}$ Among the genes that were altered in both neuronal cell lines and neurons isolated from MS brains were the SPGs (SPG4, SPG7, and SPG20). ${ }^{12}$ Various mutations in the SPGs result in multiple forms of the human disease hereditary spastic paraparesis (HSP), which is clinically similar to progressive forms of MS. ${ }^{133}$ Taken together, we determined it was necessary to resolve whether these clinically relevant genes bound hnRNP A1.

To evaluate which RNAs might theoretically bind hnRNP A1, we used the RNA Binding Protein Data Base (RBPDB, http://rbpdb.ccbr.utoronto.ca/). This database contains the unique RNA binding sequences that are required for RNA to bind RNA binding domains of an RBP. The user enters an RNA sequence into the RBPDB. The RBPDB scans the RNA sequence and reports out all RBPs that might bind to it, and the exact sequence and its location within the RNA sequence input. Using the RBPDB, we found that spastin (SPG4) contains a 100\% binding sequence (NM_014946, b.32833288) match with hnRNP A1's RNA binding sequence in its non-coding sequence. Coding regions tend not to bind RBPs, so that the coding sequence remains unencumbered for translation. SPG7 and SPG20 had variable degrees of RNA binding sequence alignment. Besides the RNA sequence, a number of variables contribute to RNA binding, such as the ability for RNAs to bind multiple RBPs, the quantity of the RBP present in the cells being analyzed and the strength of the binding between the RNA and the RBP. ${ }^{180}$ Thus, RNA binding to hnRNP A1 is not the only mechanism by which RNAs might be altered in this system. Each RNA has to be examined experimentally, which we did in the following experiments. hnRNP A1 RNA was used as a positive control because it previously was shown to be an RNA binding partner of hnRNP A1 as determined by CLIP analysis. ${ }^{181}$

To test whether the target RNAs bound hnRNP A1, we performed RNA immunoprecipitation. Protein A/G agarose beads were labeled with anti-hnRNP A1 antibodies to elute hnRNP A1 protein as well as bound RNA. In parallel, protein A/G agarose beads were labeled with isotype-matched IgG (control). SK-N-SH lysates were incubated with the antibody-bound beads. Bound protein-RNA complexes were eluted with elution buffer. The eluents were then run on Western blot (Figure 4-2). Each lane was probed for hnRNP A1 protein to prove specificity of the elution of hnRNP A1 from the immunoprecipitation compared to the IgG control (Figure 4-2A). After determining the specificity of the immunoprecipitation, RNA was extracted from the eluents using the RNA-Stat 60 protocol. ${ }^{182}$ Following first strand synthesis, the cDNA was used for quantitative RT-PCR to determine if the target RNAs chosen from the microarray data 
A.

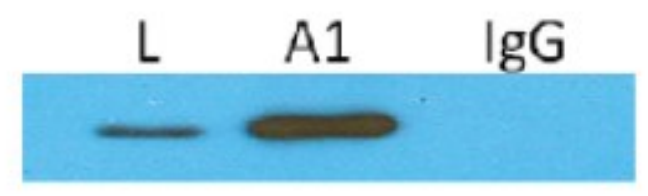

\section{B. Gene Expression of hnRNP A1 bound $\mathrm{mRNA}$}

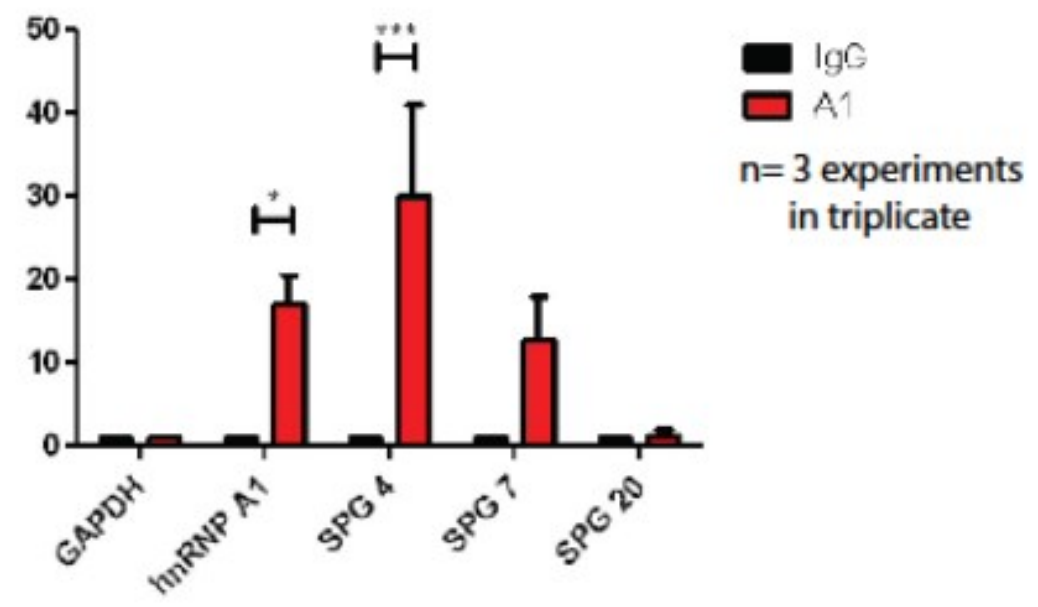

\section{Figure 4-2. RNA immunoprecipitation.}

SK-N-SH cells lysates were incubated with protein A/G agarose beads labeled with antibodies to either hnRNP A1 (ab4791) or IgG (Millipore 12-370). Proteins and any bound RNA partners were eluted from the beads. A. Protein eluents were run on $10 \%$ Tris-glycine gels for Western blotting. Gels were transferred to nitrocellulose membranes and were probed for hnRNP A1 protein (Millipore 4B10 05-1521). L= Untouched SK-N-SH lysate, A1 $=\mathrm{hnRNP} A 1$ bound agarose beads eluent, $\mathrm{IgG}=\mathrm{IgG}$ bound agarose beads eluent. The blots show that hnRNP A1 was present in the lysate (L) and the anti-hnRNP A1 IP (A1) but not in the control $\operatorname{IgG} \operatorname{IP}(\operatorname{IgG})$. B. RNA was isolated from protein eluent for each group using the RNA-stat 60 protocol. cDNA was utilized for RT-PCR probing for genes of interest. Values within groups were normalized to GAPDH. hnRNP A1 RNA was utilized as a positive control. Results revealed that hnRNP A1, SPG4, and SPG7(n.s.) are RNA binding partners of hnRNP A1. Data was analyzed using student t-test utilizing SEM. ${ }^{*} \mathrm{P}<0.05,{ }^{*} \mathrm{P}<0.01$. 
were present, indicative of their binding to hnRNP A1 protein. GAPDH was used as a quality control to normalize each group. Our results revealed that RNA of hnRNP A1, SPG4 and to a lesser extent, SPG7 bound hnRNP A1 (Figure 4-2B). In contrast, SPG20 did not bind to hnRNP A1 (Figure 4-2B).

\section{Anti-hnRNP A1-M9 Antibodies Alter SPG4 and SPG7 Protein Levels by Western Blot}

In previous studies, we showed that exposure of anti-hnRNP A1 antibodies to SK$\mathrm{N}-\mathrm{SH}$ neuronal cells in culture caused mislocalization of endogenous hnRNP A1, from a predominantly nuclear, to an equal nuclear to cytoplasmic distribution. ${ }^{1}$ In the present study, we showed that anti-hnRNP A1 antibodies localized to SGs within the neuronal cytoplasm. This suggests that the anti-hnRNP A1 antibodies targeted hnRNP A1 protein in the cytoplasm, which resulted in SG formation. Because mislocalization of RNA binding proteins from the nucleus is associated with changes in RNA metabolism (including translation), we set out to determine whether anti-hnRNP A1 antibodies caused changes in the RNA expression of RNAs bound to hnRNP A1 protein. ${ }^{183}$ Specifically, we hypothesized that protein levels of hnRNP A1's RNA binding partners (hnRNP A1, SPG4 and SPG7) would be altered whereas RNAs that did not bind hnRNP A1 (SPG-20) would not. Additionally, since anti-hnRNP A1 antibodies and normal IgG both initiated SG formation and both colocalized within SGs, we wanted to determine if anti-hnRNP A1 antibodies caused specific downstream effects (i.e. altered translation) upon the bound RNAs compared to isotype control IgG. We performed these studies by adding $8 \mathrm{ug} / \mathrm{ml}$ of anti-hnRNP A1-M9 antibodies or isotype-matched control IgG, to SK$\mathrm{N}-\mathrm{SH}$ neuronal cells. These data were compared to untreated SK-N-SH neuronal cells run in parallel. Cells were incubated with antibodies for 48 hours in complete growth media. Following the incubation period, cells were lysed and proteins extracted for Western blotting. Beta-actin was used as a control for protein expression. There was no difference in beta-actin expression between the three groups (Figure 4-3). Western blots showed that the anti-hnRNP A1 antibodies had a small effect upon hnRNP A1 protein levels and a profound reduction of SPG4 and SPG7 protein levels (Figure 4-3). There was no change in SPG20 protein levels, whose RNA did not bind hnRNP A1 (Figure 4-3). Taken together, these data indicate that in contrast to control IgG (which also localized to SGs), anti-hnRNP A1 antibodies specifically altered the translation of RNAs that bind hnRNP A1. It is likely that these downstream protein effects are largely due to the specificity of anti-hnRNP A1 antibodies for M9, hnRNP A1's nucleocytoplasmic transport domain.

\section{Aim 2 Conclusions}

Autoantibodies to various myelin as well as neuronal antigens have been implicated in the pathogenesis of MS. ${ }^{12,100-107,109,112-114,177}$ Antibodies produced against myelin antigens such as MOG, MBP, MAG, and PLP have been associated with demyelination in patients with MS, however some of these studies have been 


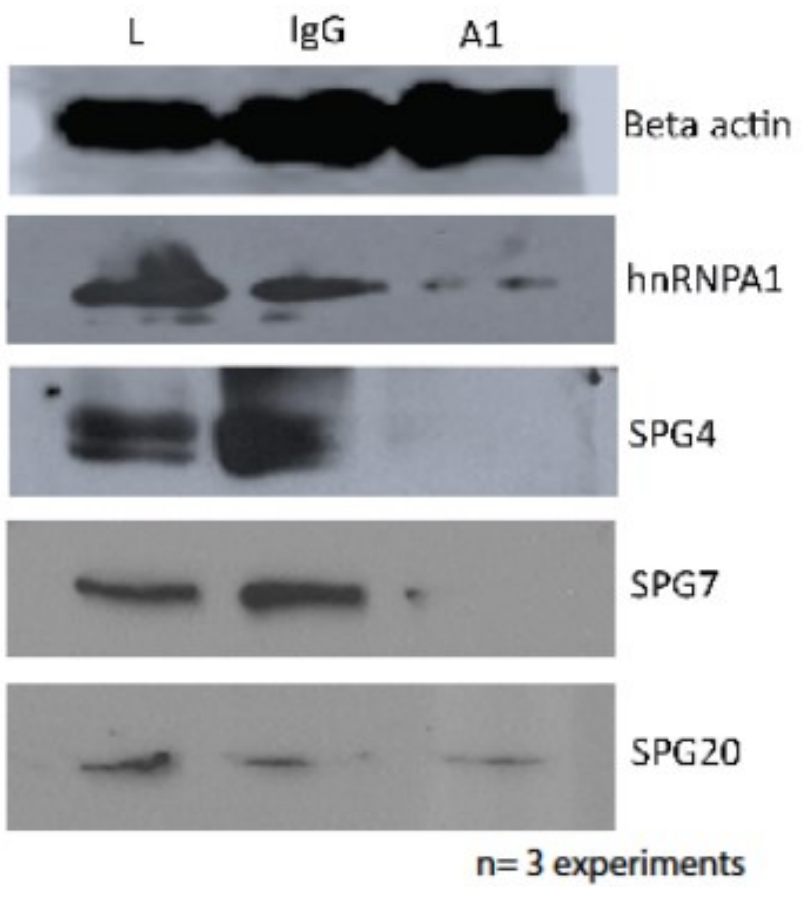

Figure 4-3. Anti-hnRNP A1 antibodies alter protein levels as measured by Western blot.

SK-N-SH cells were cultured and treated with anti-hnRNP A1 antibodies or control IgG. Following a 48 hour incubation, cells were lysed and protein lysate was run on $10 \%$ Trisglycine gels for Western blot analysis. Gels were transferred to nitrocellulose membranes, blocked, and probed for Beta Actin (control), hnRNP A1, SPG4, SPG7, and SPG20. Results revealed hnRNP A1, SPG4, and SPG7 had depleted protein values in anti-hnRNP A1-M9 antibody treated cells compared to controls. (L - lysate). 
contradictory. ${ }^{12,100-107,109,177}$ Furthermore, experiments involving antibodies to neuronal antigens such as NF-L and NF-186 suggest their role in neurodegeneration and possible disease markers of clinical progression..$^{12,112-114}$

Previous studies have shown that patients with MS produce antibodies to hnRNP A1 while healthy controls as well as patients with other neurologic disease have no titer specific to hnRNP A1. ${ }^{1,2,12,95,97,99}$ hnRNP A1 is an RBP highly expressed in neurons responsible for trafficking of various mature mRNA from the nucleus to the cytoplasm for translation. hnRNP A1 has a C-terminal region termed 'M9', which is its NES/NLS responsible for the nuclear - cytosolic trafficking of hnRNP A1.,2,11,12,94,95,97,99 The antibodies produced by MS patients are specific to this M9 region. ${ }^{1,2,11,12,95,99}$ Our previous studies have shown that endogenous hnRNP A1 was redistributed from a primarily nuclear localization to an equal nuclear/cytoplasmic mislocalization due to the specificity of the anti-hnRNP A1-M9 antibodies. ${ }^{1}$ Additionally, our data shows that the specificity of the anti-hnRNP A1 antibodies resulted in markers of neurodegeneration including decreased cellular ATP levels and increased apoptosis of neurons ${ }^{1}$. The current studies show that anti-hnRNP A1 antibodies cause some cellular stress resulting in formation of SGs to which the antibodies colocalize. Additionally, we demonstrate that SPG4 and SPG7 are RNA binding partners of hnRNP A1. Furthermore, we determined that anti-hnRNP A1 specific antibodies have profound effects upon specific RNA binding partners of hnRNP A1 protein. Specifically, we showed that SPG4 and SPG7 protein expression are markedly reduced due to the specificity of anti-hnRNP A1 antibodies compared to isotype control IgG.

Taken together, these studies suggest a mechanism by which autoantibodies cause neurodegeneration. One of hnRNP Al's many roles in RNA metabolism ('ribostasis') is to bind RNA, transport RNA from the nucleus to the cytoplasm, where it undergoes translation. ${ }^{184} \mathrm{hnRNP} \mathrm{A} 1$ is then quickly transported back to the nucleus. Thus, under normal cellular homeostasis, hnRNP A1 is predominantly localized to the nucleus. Our data indicate that the sequence of events by which anti-hnRNP A1 antibodies cause neurodegeneration is as follows. First, anti-hnRNP A1 antibodies enter neurons by clathrin-mediated endocytosis. Second, the anti-hnRNP A1 antibodies bind M9, which is hnRNP A1's nucleocytoplasmic shuttling domain. The binding interrupts the normal transport of hnRNP A1 into the nucleus, thus hnRNP A1 is mislocalized to the cytoplasm, which results in cellular stress and SG formation. The anti-hnRNP A1 antibodies localize to SGs, where they cause translational repression of SPG4 and SPG7, two clinically relevant RNAs, which now have been shown to bind hnRNP A1. Thus, anti-hnRNP A1 antibodies bind M9, which causes mislocalization of hnRNP A1 to the neuronal cytoplasm, SG formation, translational repression of RNA bound to hnRNP A1 and subsequent neurodegeneration. ${ }^{1,2,11,12,95,99}$

It is important to note that mutations in the genes affected (SPG4, SPG7) result in various forms of HSP. ${ }^{133}$ HSP is a genetically inherited spastic disorder similar clinically to MS. ${ }^{133}$ Mutations in SPG4 account for the most common autosomal dominant form of HSP. ${ }^{133}$ Additionally, SPG7 is a gene associated with mitochondrial function, specifically with mitochondrial respiratory chain complexes I and IV. ${ }^{\mathbf{1 8 5}}$ It was shown 
that patients with HSP resulting from mutations in SPG7 showed a significant decrease in mitochondrial respiratory chain complexes I and IV. ${ }^{\mathbf{1 8 5}}$ Previously we have shown depleted ATP levels in vitro due to the addition of anti-hnRNP A1 antibodies. In this article we show that anti-hnRNP A1 antibodies alter protein levels of SPG7 in vitro. In concurrence with the studies in HSP patients with depleted mitochondrial respiratory chain complexes, our data suggests that altered SPG7 levels due to anti-hnRNP A1 antibodies might act in a similar mechanism to the SPG7 mutations seen in patients with autosomal recessive HSP.

In summary, we have shown that anti-hnRNP A1 antibodies cause cellular stress resulting in SGs to which the antibodies colocalize. Additionally, we determined that SPG4, and SPG7 are RNA binding partners of hnRNP A1, which are translationally repressed due to the specificity of anti-hnRNP A1 antibodies. These studies emphasize the importance of autoantibodies to non-myelin antigens in the pathogenesis of MS and shed insight into a possible mechanism of how autoantibodies to hnRNP A1 cause changes in neuronal function which ultimately leads to neurodegeneration. The work presented in AIM 2 builds upon the work previously displayed in AIM 1. We have shown further trafficking information concerning antibodies following penetration of neuronal cells. We have shown that antibodies not only cause stress resulting in stress granules, but also that the antibodies colocalize to stress granules. Furthermore, we have further exhibited that the anti-hnRNP A1 antibodies have a specific effect that is not present in the IgG treated cells. Anti-hnRNP A1 antibodies specificity leads to translational repression of the SPGs (SPG4 and SPG7) which could expose a possible mechanism of cell death. The data combined from the first two AIMs provides ample ground work set for the final AIM to make the transition into a murine model in order to determine if antibody specific neurodegeneration translates from an in vitro model to an in vivo model of MS. 


\section{CHAPTER 5. ANTI-HNRNP A1 ANTIBODIES INCREASE NEURODEGENERATION IN EXPERIMENTAL AUTOIMMUNE ENCEPHALOMYELITIS (EAE), AN ANIMAL MODEL OF MS}

\section{Introduction}

In vitro studies have shown that anti-hnRNP A1 antibodies whose epitope overlapped the immunodominant epitope recognized by MS patients caused neurodegeneration in neuronal cell lines. In this study, we show that injection of antihnRNP A1 antibodies in mice with experimental allergic encephalomyelitis (EAE) worsen clinical disease, alter the clinical phenotype of the mice and cause neurodegeneration in specific central nervous system (CNS) pathways. One pathway affected was the ventral spinocerebellar tract (VSCT), which showed preferential neurodegeneration compared to control groups consistent with 'dying back' axonal degeneration. Taken together, these data suggest that antibodies contribute to neurodegeneration in immune-mediated disease of the CNS.

Neurodegeneration, including neuronal and axonal damage, has been shown to contribute to the pathogenesis of multiple sclerosis (MS). ${ }^{7,9,13,186-188}$ Manifestations of neurodegeneration have been observed using neuroradiological, neuropathological and animal studies of MS. For example, magnetic resonance imaging (MRI) brain images of MS patients show axonal damage as well as brain and spinal cord atrophy, which correlate with neurological disability. ${ }^{189,190}$ Pathologically, accumulation of amyloid precursor protein (APP) ${ }^{15,191}$ and staining for non-phosphorylated neurofilament (SMI32) ${ }^{9}$ (both markers of axonal injury) showed that axonal damage is a major component of MS lesions. ${ }^{9,187,192,193}$ Importantly, several studies have shown the presence of neurodegeneration throughout the disease course of MS, not just during latter progressive phases of disease, as was originally thought. ${ }^{187}$ In experimental allergic encephalomyelitis (EAE), an animal model of MS, data indicate that (like in MS) neuronal and axonal damage are present throughout the course of the disease, and thus contribute to the neurologic disability displayed by the animals. ${ }^{148,194,195}$

Antibodies that target neuronal and axonal antigens are one of several proposed mechanisms that might contribute to neurodegeneration in MS and EAE. ${ }^{11}$ For example, MS patients develop antibodies to the 'axolemma-enriched fraction', neurofilaments (NFs) and neurofascin. ${ }^{196-200}$ In mice, immunization with NF-L protein resulted in spastic paraparesis concurrent with spinal cord axonal degeneration. ${ }^{200,201}$ The mice developed a pro-inflammatory $\mathrm{T}$ - cell response, and importantly, also developed antibodies to NF-L and IgG deposits within axons of spinal cord lesions. ${ }^{200,201}$ Further, MS patients were found to make antibodies to neurofascin ${ }^{112,202}$, a protein with an isoform present on axons at the nodes of Ranvier. ${ }^{112,202}$ Application of these antibodies to hippocampal slice cultures inhibited axonal conduction. Following induction of EAE with myelin oligodendrocyte glycoprotein (MOG) specific T-cells, the addition of anti-neurofascin antibodies augmented disease. ${ }^{112,202}$ 
MS patients (in contrast to healthy controls) have also been found to have antibodies to hnRNP A1, a ubiquitously expressed RNA binding protein (RBP) that is over expressed in neurons. ${ }^{11,12,94,95,192,193}$ Importantly, RBPs including hnRNP A1 regulate RNA metabolism and dysfunctional RBPs have been shown to cause neurodegeneration in amyotrophic lateral sclerosis (ALS) and frontotemporal lobe dementia (FTLD). ${ }^{132,184,203}$ The immunodominant epitope of hnRNP A1 recognized by MS IgG was experimentally determined to include 'M9', hnRNP A1's nucleocytoplasmic shuttling domain, which is required for its transport into and out of the nucleus to the cytoplasm. ${ }^{11,12,204,205}$ In an in vitro model of neurodegeneration, anti-hnRNP A1 antibodies that overlap the MS 'M9' immunodominant epitope caused neurodegeneration (as shown by Fluoro-jade $\mathrm{C}$ staining), apoptosis, and changes in gene expression related to hnRNP A1 function and the clinical phenotype of MS patients (ie. spastic paraparesis and ataxia). ${ }^{11,12,192}$ In separate in vitro experiments, anti-hnRNP A1 antibodies were found to enter neurons by utilizing endocytosis, a mechanism identical to antibodies isolated from ALS patients and monoclonal antibodies directed at Tau protein. ${ }^{11,206-208}$ Further, the anti-hnRNP A1 antibodies caused the mis-localization of hnRNP A1 from predominantly nuclear to an equal nuclear/cytoplasmic distribution suggesting that the anti-hnRNP al antibodies bound M9 and disrupted hnRNP A1's normal trafficking between the nucleus and cytoplasm. ${ }^{11,206}$

Considering these data, we hypothesized that anti-hnRNP A1 antibodies might promote neurodegeneration in vivo. We tested this hypothesis by injecting anti-hnRNP A1 antibodies in C57BL/6J mice with MOG-induced EAE. MOG-C57BL/6J EAE mice develop chronic disease with very little variation once peak disease has been achieved. A recent study meticulously mapped the timing of neurodegeneration of the entire central nervous system (CNS) using Fluoro-jade C, a marker of degenerating neural elements. ${ }^{148}$ We paralleled this paradigm and found that the anti-M9 antibodies caused neurodegeneration in several neuronal pathways with a strong clinical correlation with signs and symptoms of MS patients.

\section{Results}

\section{Anti-hnRNP A1-M9 Antibodies Exacerbate MOG35-55 Induced EAE}

Following induction of EAE, mice were monitored daily for early clinical signs (limp tail) of EAE. Upon first clinical signs of EAE, mice were injected with PBS (injection control), anti-mouse IgG2b (isotype control), or mouse anti-hnRNP A1 antibodies (100 ug ip. every other day for a total of three injections). Untouched nonEAE C57BL/6J mice were used as a non-disease control. Anti-hnRNP A1 antibody treated mice developed statistically worse clinical disease compared to all other groups (Figure 5-1). Interestingly, anti-hnRNP A1 treated mice developed a more spastic gait compared to all other groups. In a typical experiment, $50 \%$ of the anti-hnRNP A1 injected animals were spastic compared to only $10 \%$ of the mice in other EAE groups. Furthermore, several anti-hnRNP A1 mice had to be euthanized prior to the end of the 


\section{Combined Classic EAE with Ab Addition}

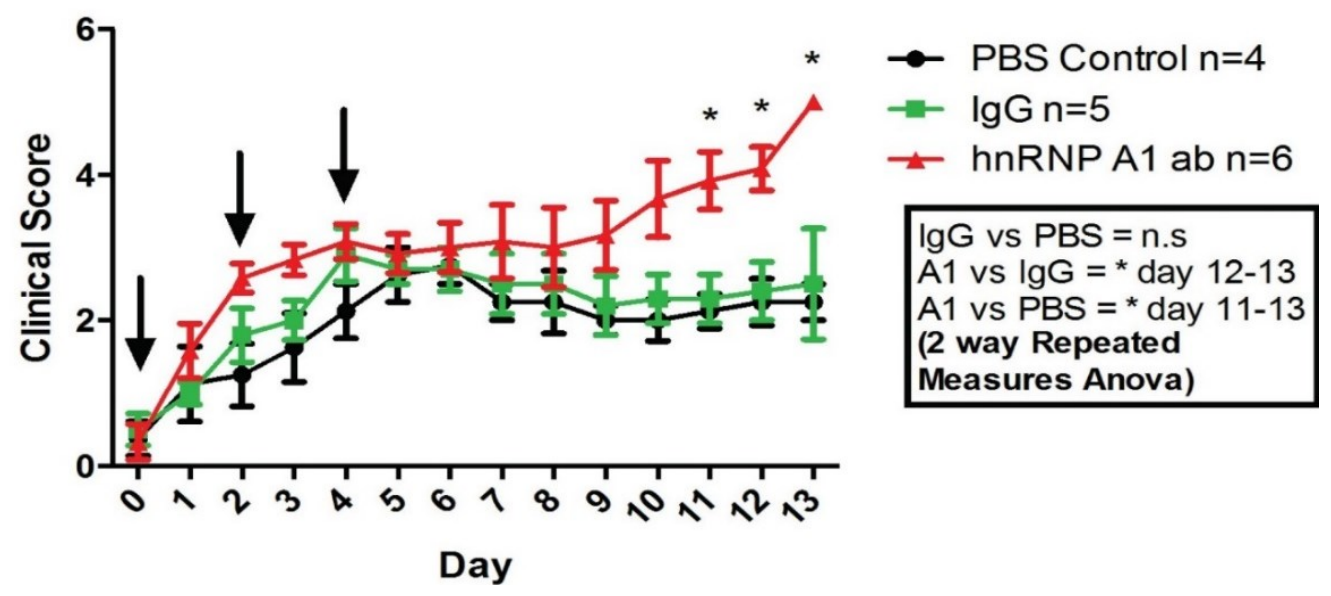

Figure 5-1. Clinical scores of EAE mice.

EAE was induced in C57/B16 mice using MOG35-55/CFA and pertussis toxin. On first signs of clinical symptoms, mice were given $100 \mu \mathrm{g}$ injections of PBS, IgG (MABC006), or anti-hnRNP A1 antibody (04-1469). Injections were given every other day for a total of three injections. EAE mice were scored daily from disease onset. Clinical scoring was adapted from 'Appendix A- EAE scoring guide' found in the protocol of the Active Induction EAE Kit from Hooke Laboratories (Hooke cat no. EK-2110). Generally speaking, the scoring was as follows: 1- limp tail, 2- hind limb weakness/wobbly gait, 3hind limb paralysis, 4- hind limb paralysis/weakness of front limbs, 5- moribund. Animals were scored clinically throughout the study. Anti-hnRNP A1-M9 antibody treated mice developed increased spasticity and paralysis compared to control groups. Quantification was performed in Graphpad Prism software. Statistical significance was analyzed by two-way repeated measures ANOVA using $\mathrm{SEM}$. $* \mathrm{P}<0.05, * * \mathrm{P}<0.01$. 
study due to the severity of the spasticity and clinical disease, whereas mice in the other two groups did not commonly reach this severe disease state. Additionally, in contrast to all other groups, some of the anti-hnRNP A1 mice developed tail lesions (30\% of antihnRNP A1 EAE compared to $0 \%$ of PBS or isotype control EAE). Previously published studies have associated tail lesions in EAE with increased disease severity and immune responses ${ }^{209}$. Taken together, these data indicate that the anti-hnRNP A1 antibodies contributed to worsened clinical disease and a change in the phenotype of the mice (spastic compared to flaccid paralysis).

\section{Anti-hnRNP A1-M9 Antibodies Cause Increased Levels of Neurodegeneration of Specific CNS Pathways in EAE}

Upon observing that anti-hnRNP A1 antibodies worsened EAE, we hypothesized that the augmented clinical progression might be related to changes in neurodegeneration in the CNS. To examine this, we stained the brains and spinal cords of three animals from each group with Fluoro-jade $\mathrm{C}$, a marker of degenerating neural elements. Fluorojade $\mathrm{C}$ has been used previously to map neurodegeneration in the identical active $\mathrm{MOG}_{35-55}$ induced EAE protocol utilized in these studies. We compared data between groups and used the map of Fluoro-jade $\mathrm{C}$ neurodegeneration in EAE published by Brown and Sawchenko as reference. ${ }^{148}$

There was very minimal background Fluoro-jade $\mathrm{C}$ staining of non-neural elements in spinal cords or brains in untouched (non-EAE) animals as previously described (Figures 5-(2-5), 'control'). ${ }^{148}$ In the spinal cords of the three EAE groups (PBS, isotype control IgG, anti-hnRNP A1), we found a similar distribution of staining (Figure 5-2). Staining (white punctates) dominated in the ventral spinocerebellar tracts (VSCT) at all levels of the spinal cord, with lesser amounts in the dorsal spinocerebellar tracts (DSCT). There was also a modest degree of staining in the posterior columns and throughout the grey matter of the spinal cord (Figure 5-2). There were no differences in Fluoro-jade $\mathrm{C}$ fluorescent intensity between the three EAE groups in the spinal cord (VSCT as an example, Figure 5-2).

Examination of the brainstems of these animals revealed neurodegeneration in the VSCT, trapezoid body, spinal tract of the trigeminal nerve, inferior cerebellar peduncle and other structures within the brainstem as described previously (Figure 5-3A and Table 5-1). ${ }^{148}$ There was an absence of staining in the superior cerebellar peduncle. There was neurodegeneration in the cerebellar peduncles that continued to include the pyramids (not shown) in all EAE induced animals. The cerebellar peduncle data parallels that of Brown and Sawchenko (Table 5-1) ${ }^{148}$, but the pyramidal data differs in that there was no reported Fluoro-jade $\mathrm{C}$ staining. Both involve the corticospinal system and these differences may be related to the length of the experiments (which were about a week longer than the experiments by Brown et al). By comparing the neurodegeneration between EAE groups, it was determined that there was an increase in Fluoro-jade C staining in one specific tract, the VSCT, of the anti-hnRNP A1 antibody treated mice compared to the other EAE mice. The distal VSCT of the brainstem as it entered the 


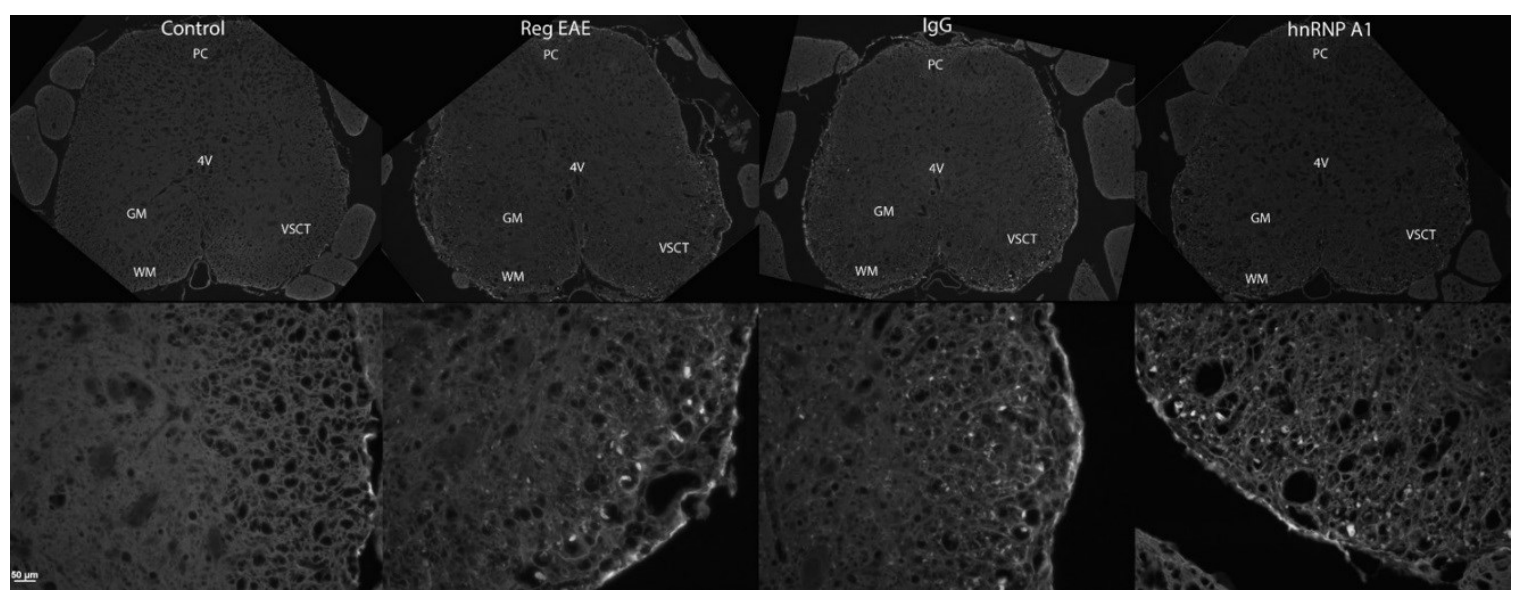

Figure 5-2. Fluoro-jade $C$ staining of the spinal cord.

Paraffin embedded brain sections from the lumbosacral region of the spinal cord were cut into $10 \mu \mathrm{m}$ sections. Sections were stained for neurodegeneration using the Fluoro jade $\mathrm{C}$ neurodegeneration marker. Zoomed images were taken from the ventral spinocerebellar tract cells of origin. 
A.

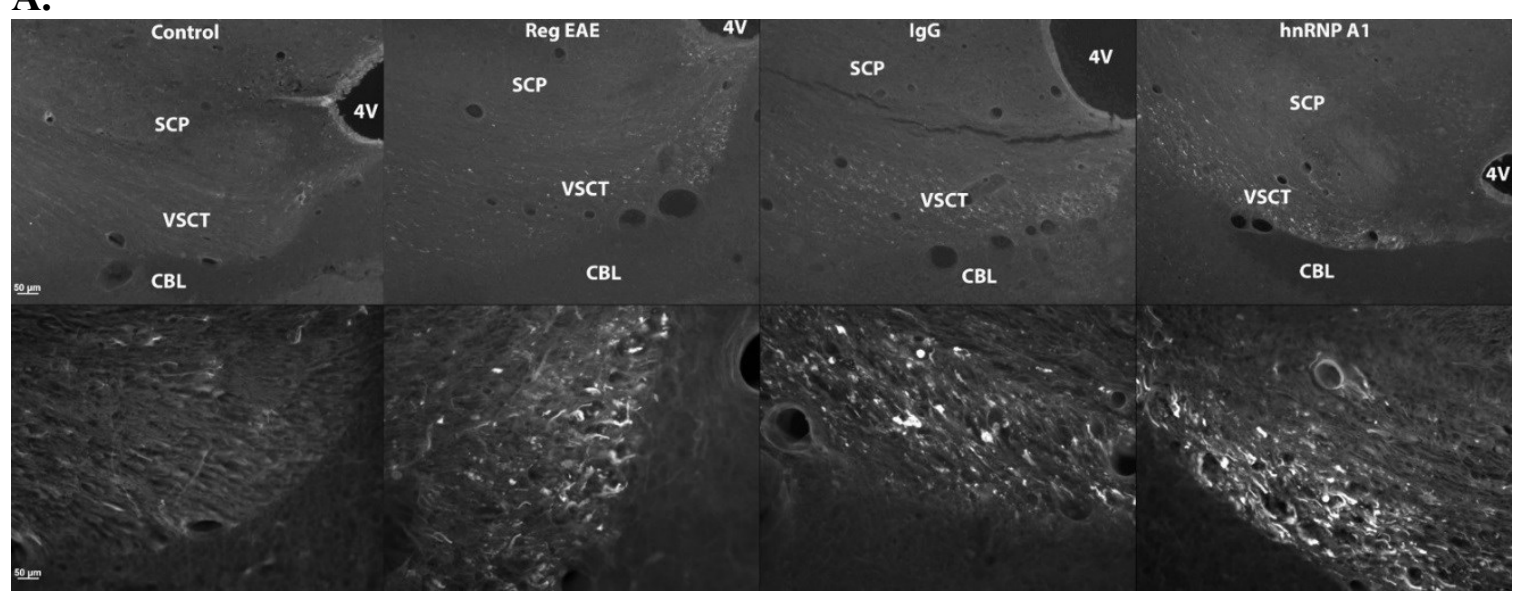

B.

Ventral Spinocerebellar tract Neurodegeneration

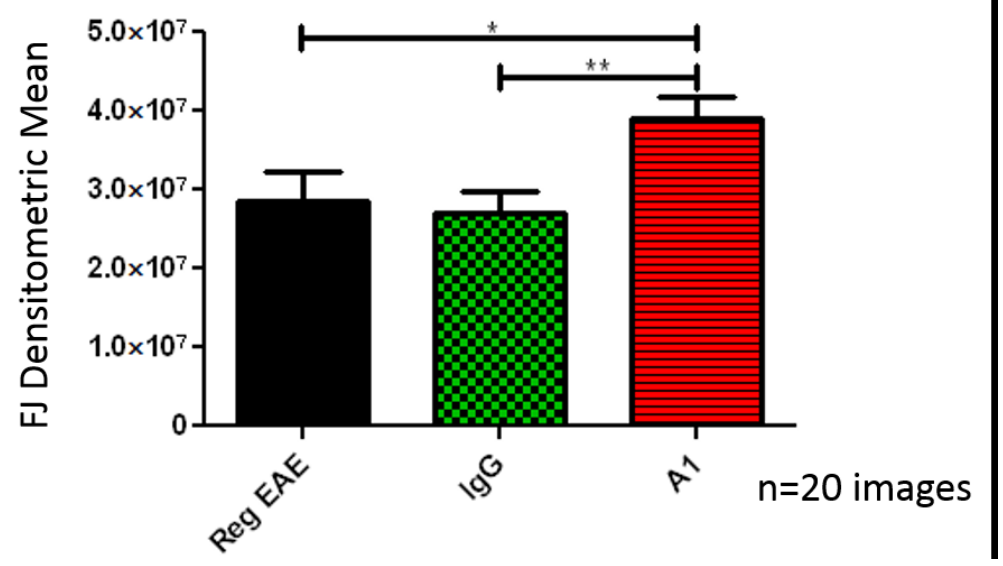

Figure 5-3. Fluoro-jade $\mathrm{C}$ staining of the ventral spinocerebellar tract of the brain stem.

A. Fixed frozen brain sections from the brain stem were cut using a sliding microtome. Sections were cut at $30 \mu \mathrm{m}$. Sections were stained for neurodegeneration using Fluoro jade $\mathrm{C}$ neurodegeneration marker. Zoomed image is taken from the area of the ventral spinocerebellar tract labeled with the arrow. B. Quantification of neurodegeneration was performed on 20 total images obtained from 3 animals per experimental group (20 images per condition). Mean fluorescence intensity was calculated using image $J$ software. Mean fluorescence intensities were compared using the student $t$-test using SEM. $* \mathrm{P}<0.05, * * \mathrm{P}<0.01$. 
Table 5-1. Areas of neurodegeneration in an antibody augmented model of EAE.

\begin{tabular}{lcc}
\hline Area of brain & $\begin{array}{c}\text { Neuro- } \\
\text { degeneration }\end{array}$ & $\begin{array}{c}\text { Increased } \\
\text { neurodegeneration } \\
\text { in A1 mice }\end{array}$ \\
\hline Brain Stem Tracts & + & + \\
Ventral Spinocerebellar Tract & + & - \\
Trapezoid Body & + & - \\
Spinal Tract of the Trigeminal Nerve & + & - \\
Inferior Cerebellar Peduncle & + & - \\
Pyramids & - & - \\
Superior Cerebellar Peduncle & & - \\
Cerebellum & - & - \\
Dentate Nucleus & - & + \\
Interposed Nucleus & - & + \\
Fastigial Nucleus & + & - \\
Deep White Matter (arbor vitae) & & - \\
Cerebral Cortex & + & \\
Corpus Callosum & & - \\
\hline
\end{tabular}


cerebellum parallel to the superior cerebellar peduncle showed increased neurodegeneration in anti-hnRNP A1 mice (Figure 5-3A and Table 5-1). Quantitative analysis (10 images from each of 3 mice per group, total of 30 images per group) of Fluoro-jade $\mathrm{C}$ fluorescence using Image $\mathrm{J}$ software confirmed an increase in neurodegeneration in the anti-hnRNP A1 mice compared to all other groups (Figure 53B). The cells of origin of the VSCT are admixed with other neurons within layers VII, VIII and IX of the gray matter of the lumbosacral spinal cord. ${ }^{210-215}$ Some of the neurons are grouped into partially defined nuclei within layer VII including spinal border cells (SBCs), and the lumbar and sacral pre-cerebellar nuclei. ${ }^{214,216}$ Axons from these neurons immediately cross in the spinal cord at their level of origin in the ventral white matter. These projections make up the VSCT, which continues up the spinal column through the lateral funiculi, followed by the brainstem and then enters the cerebellum with the superior cerebellar peduncle, where its projections then enter the deep white matter of the cerebellum. ${ }^{210-215}$ With this in mind, we next examined the cerebellum for changes in neurodegeneration between the three EAE groups.

We observed large quantities of neurodegeneration in the deep white matter (arbor vitae) of the cerebellum (Figure 5-4 and Table 5-1). Interestingly, both in this study as well as the study by Brown and Sawchenko ${ }^{148}$, this area contains some of the most intense Fluoro-jade C staining of the CNS in EAE. Furthermore, in concordance with the data presented by Brown and Sawchenko, we also saw a lack of neurodegeneration in efferent pathways of the cerebellum including Purkinje cells and the interposed, fastigial, and dentate nuclei (Table 5-1). ${ }^{148}$ Comparison of all EAE groups as well as healthy controls revealed that there was increased neurodegeneration in the deep white matter (arbor vitae) of the cerebellum of anti-hnRNP A1 antibody treated animals compared to all other EAE groups (Figure 5-4A and Table 5-1). This finding was quantified and confirmed using image $\mathrm{J}$ software, which showed a statistically significant increase in Fluoro-jade C staining in the anti-hnRNP A1 group (Figure 5-4B). There was minimal staining of the granule layer in the cerebellum, none of which included cell bodies.

After investigating the brain stem and the cerebellum, we examined the cerebral cortex for changes in neurodegeneration. We observed identical patterns of neurodegeneration as published previously. ${ }^{148} \mathrm{We}$ also witnessed neurodegeneration in the corpus callosum of all EAE induced mice (Table 5-1). ${ }^{148}$ Furthermore, we found that there was increased neurodegeneration in the genu of the corpus callosum of the antihnRNP A1 antibody treated mice compared to all other groups (Figure 5-5A and Table 5-1). Again, this observation was confirmed by quantitative imaging (Figure 5-5B).

\section{Conclusions for Aim 3}

Our data indicate that in EAE, an animal model of MS, antibodies to the 'M9' sequence of hnRNP A1 (compared to PBS and isotype matched IgG2b controls) caused: (1) worsened clinical disease, (2) an altered clinical phenotype (spastic paraparesis), (3) increased levels of neurodegeneration in the distal projection of the VSCT in the deep 


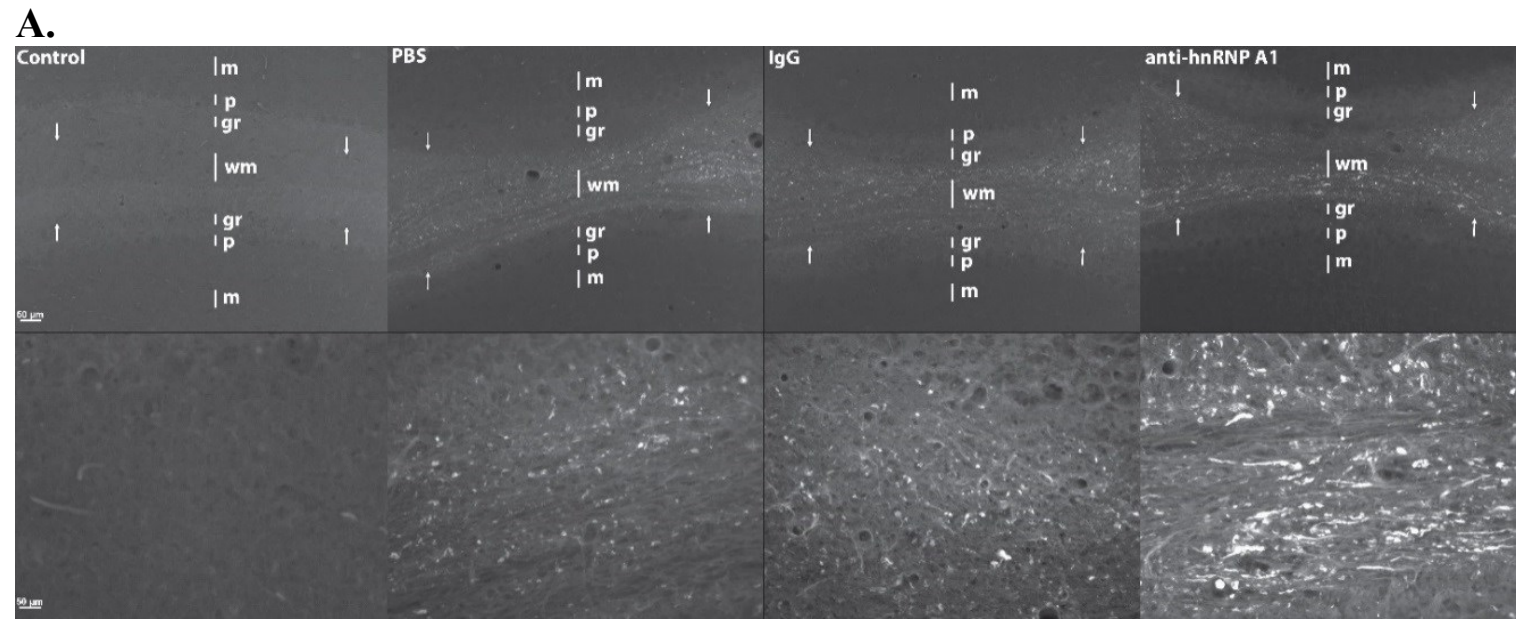

B.

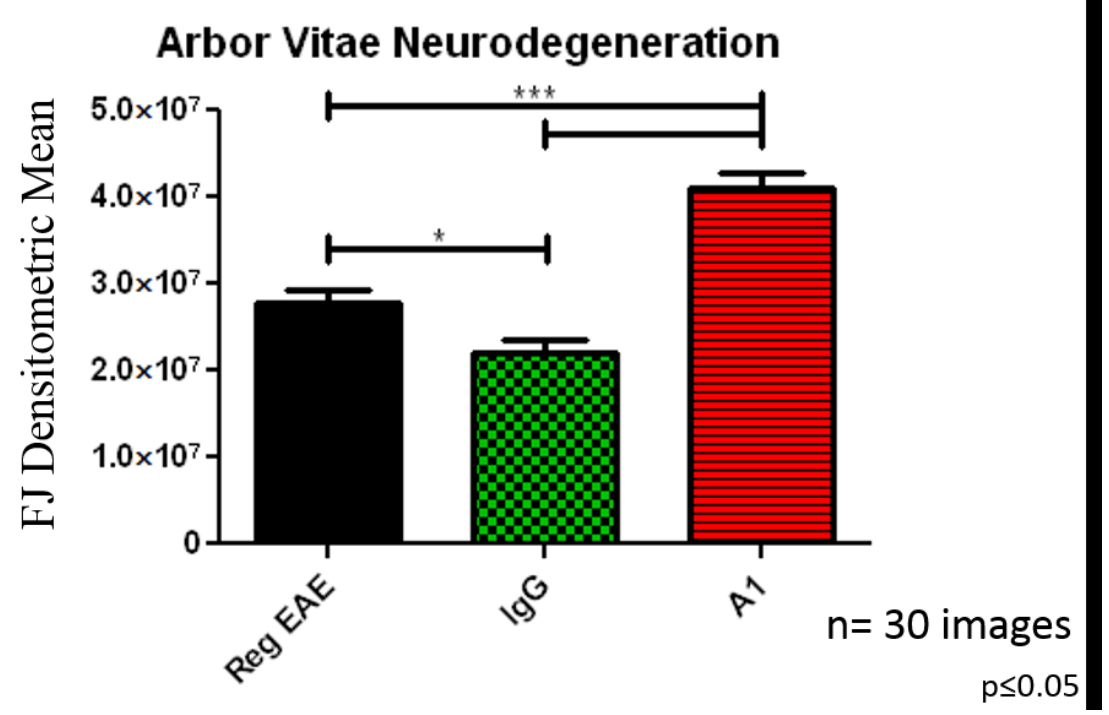

Figure 5-4. Fluoro-jade $C$ staining of the deep cerebellar white matter.

A. Fixed frozen brain sections from the cerebellum were cut using a sliding microtome. Sections were cut at $30 \mu \mathrm{m}$. Sections were stained for neurodegeneration using Fluoro jade $\mathrm{C}$ neurodegeneration marker. Zoomed image is taken from the labeled area from the center of the white matter (labeled white line). Cerebellar layers are labeled as follows: wm- white matter, gr- granular layer, p- purkinje cell layer, m- molecular layer. Arrows represent the border of the deep white matter of the cerebellum (wm). B. Quantification of neurodegeneration was performed on 10 images from 3 animals per experimental group (30 images per condition). Mean fluorescence intensity was calculated using image J software. Mean fluorescence intensities were compared using the student t-test and $\mathrm{SEM} * \mathrm{P}<0.05, * * \mathrm{P}<0.01$. 


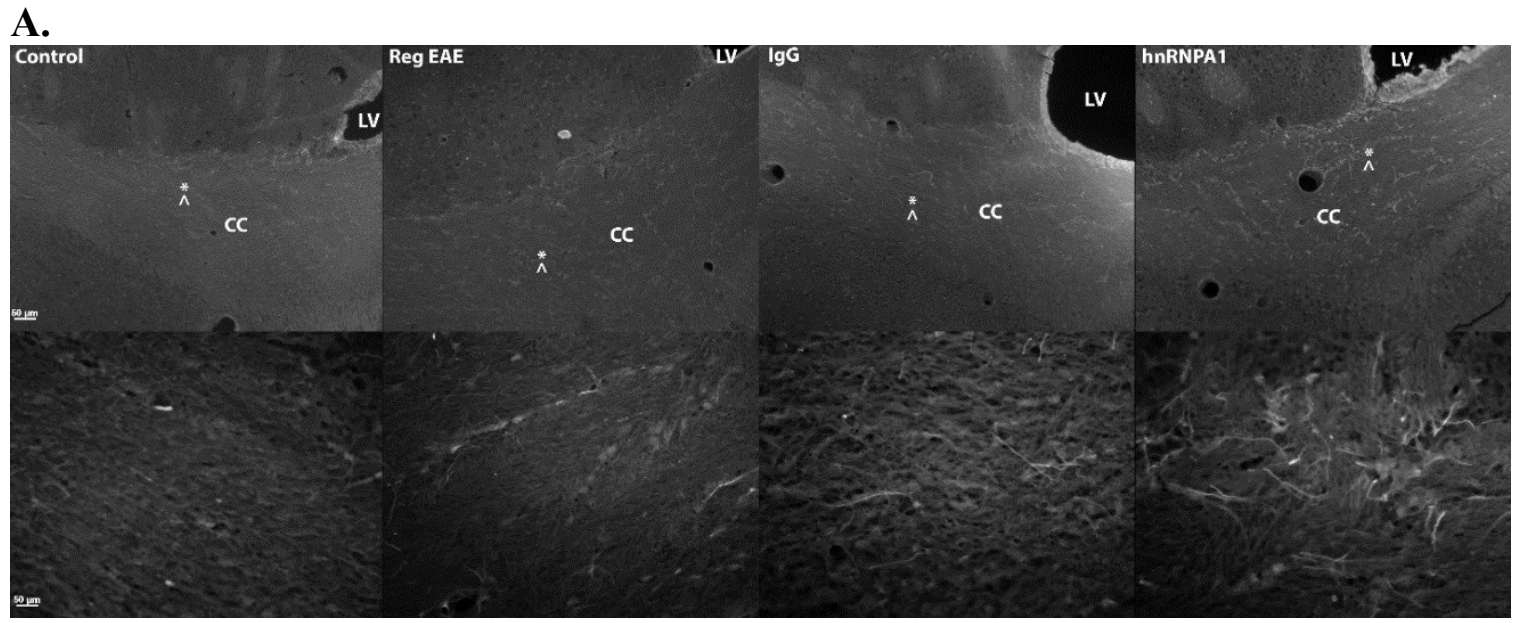

B.

\section{Corpus Callosum Neurodegeneration}

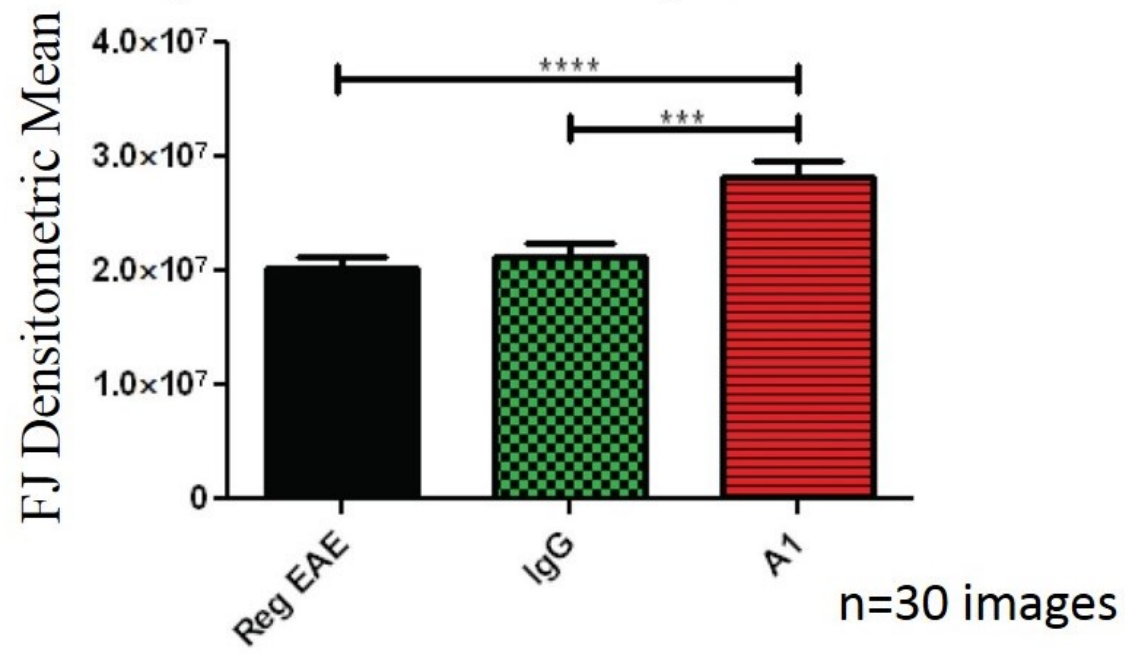

Figure 5-5. Fluoro jade $C$ staining of the corpus callosum.

A. Fixed frozen brain sections from the cerebral cortex were cut using a sliding microtome. Sections were cut at $30 \mu \mathrm{m}$. Sections were stained for neurodegeneration using Fluoro jade $\mathrm{C}$ neurodegeneration marker. Images were taken from the genu of the corpus callosum adjacent to the lateral ventricle. Zoomed images were taken from the center of the genu marked by an asterisk adjacent to the lateral ventricle. B. Quantification of neurodegeneration was performed on 10 images from 3 animals per experimental group (30 images per condition). Mean fluorescence intensity was calculated using image $\mathrm{J}$ software. Mean fluorescence intensities were compared using the student t-test and SEM $* \mathrm{P}<0.05,{ }^{*} * \mathrm{P}<0.01$. 
white matter of the cerebellum and (4) increased levels of neurodegeneration in the genu of the corpus callosum.

We believe the results of these experiments are consistent with an antibodymediated autoimmune response to a CNS target that contributes to a 'dying back' mechanism of axonal degeneration. "Dying back," describes length-dependent, distal to proximal, progressive axonal degeneration, which occurs in toxic, metabolic and neurodegenerative diseases. ${ }^{188,217-219}$ Although there was axonal degeneration within the proximal VSCT in the spinal cord, there were no differences between control and antihnRNP A1 mice. The cells of origin of the VSCT are contained within laminae VII, VIII and IX of the lumbosacral spinal cord, thus the most proximal segments of the VSCT are in the spinal cord. ${ }^{210-215}$ Moving rostrally, neurodegeneration of the VSCT continued in both control and anti-hnRNP A1 mice in the brainstem, but again there were no differences between the groups. As the VSCT entered the cerebellum and continued in the arbor vitae (deep cerebellar white matter), the anti-hnRNP A1 mice showed significantly worse neurodegeneration than either of the control groups. These segments of the VSCT contain its most distal axons as they synapse on granule cells in the cerebellum. Considering there are no differences in proximal, but profound differences in distal segments of the VSCT, this is consistent with 'dying back' neurodegeneration (Figure 5-6). ${ }^{188,217-219}$ Importantly, the VSCT makes afferent connections to the corticospinal tract via cerebellar efferents (Figure 5-6). Considering that both cerebellar lesions and genetic mutations in diseases such as the inherited spastic ataxias cause changes in muscle tone ${ }^{220-222}$, our data suggests that neurodegeneration of the VSCT might also contribute to spasticity, which was readily apparent in the anti-hnRNP A1 antibody injected mice. These data add to the growing body of evidence that EAE is a suitable animal model to study neurodegeneration mechanisms that may be applicable to MS. ${ }^{148,194,195,223-225}$

Neurodegeneration is now believed to be the primary cause of permanent, longterm disability in MS patients. ${ }^{187,188,192,193}$ Data indicate neuronal and axonal damage in an ongoing process in MS. 7,9,13,186,187 In some patients, it begins during the relapsing, remitting phase of MS (RRMS) and continues into secondary progressive MS (SPMS). Neurodegeneration is also a common feature in MS patients without relapses (primary progressive MS (PPMS). ${ }^{187}$ Pathologically, neurodegeneration appears independent of plaque formation. ${ }^{18}$ For example, analysis of the corticospinal tract in the spinal cord of MS patients showed a significant reduction of nerve fiber density independent of cerebral plaque location. $^{226-230}$ Interestingly, like the corticospinal tract, the spinocerebellar tracts are some of the longest of the CNS. Cerebellar symptoms are common and make major contributions to disability in MS patients. ${ }^{231}$

The brains and spinal cords of MS patients show evidence of both neuronal and axonal degeneration. For instance, there is neuronal loss and apoptosis of cortical pyramidal neurons. ${ }^{232}$ In addition, MS brains display markers of axonal degeneration including axonal dystrophy, axonal spheroids, and staining for non-phosphorylated neurofilament (SMI-32). 7,9,18,186,191 These markers confirm the presence of axonal degeneration in MS, but do not implicate a specific mechanism. ${ }^{219}$ The cause of 


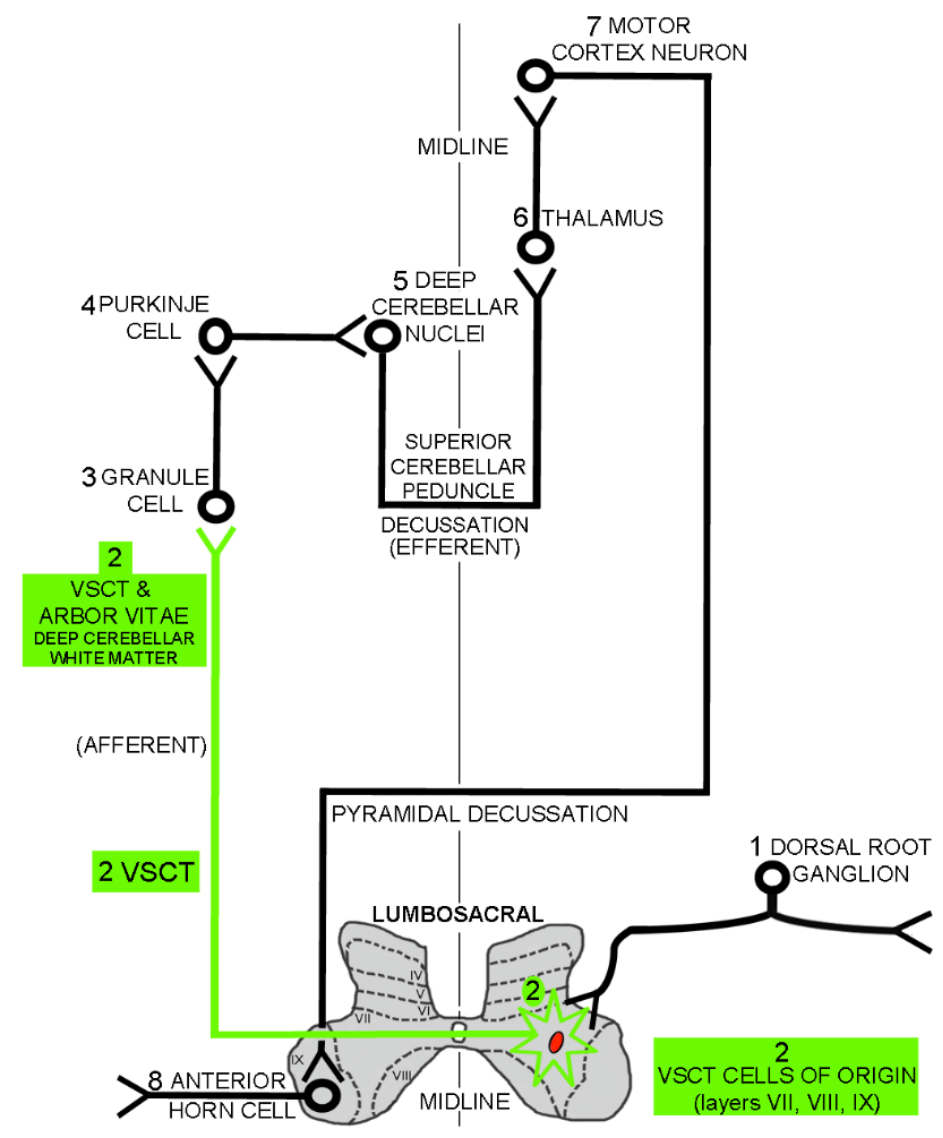

Figure 5-6. Neural pathways related to the ventral spinocerebellar tract (VSCT). (1) Dorsal root ganglia synapse on the neuronal cell bodies of (2) the VSCT, which are contained within laminae VII, VIII and IX of lumbosacral levels of the spinal gray matter. Axons of the (2) VSCT, which are preferentially damaged by the anti-hnRNP A1M9 antibodies (green), cross in the spinal cord \& pass through the deep cerebellar white matter to synapse on (3) granule cells. (3) Granule cells synapse on (4) Purkinje cells, which in turn, synapse in (5) deep cerebellar nuclei. (5) Deep cerebellar nuclei send efferent axons to (6) the thalamus, which relays information to (7) the motor cortex, which sends axons that synapse on (8) anterior horn cells in the ventral gray matter (lamina IX) of the spinal cord. 
neurodegeneration appears to be multifactorial. In MS, there is evidence of both Wallerian Degeneration (transection of axons that disconnects the neuronal cell body from its distal axon) and 'dying back' pathologies. ${ }^{188,218,233,234}$ Both involve abnormalities in axonal transport, which is demonstrated by the accumulation of APP in axons of MS brains, which occurs in the setting of dysfunctional fast axonal transport. ${ }^{7,191,219}$ In animal models, axonal degeneration results from a specific sequence of events, which include impaired axonal transport, mitochondrial dysfunction and increase in intraaxoplasmic calcium. ${ }^{218,225}$ Importantly, each of these abnormalities has been found in brains of MS patients as well as in EAE, and have been implicated in the pathogenesis of neurodegeneration. Several studies have shown that mitochondrial injury and associated oxidative damage, production of reactive oxygen species and induction of apoptosis contribute to axonal injury. ${ }^{13,17,235,236}$ Other studies implicate increased energy demands of demyelinating axons and the relative reduction of axonal ATP production, which results in a state of 'virtual hypoxia'. ${ }^{38,237}$ In addition, there is evidence that neurodegeneration might result from lack of trophic support from myelin ${ }^{8}$, inflammatory cytokines abnormal ion and sodium channel expression on axons ${ }^{9,18,238}$, and calcium accumulation in damaged axons..$^{9,237}$

Antibodies to CNS targets have been shown to contribute to neurodegeneration. ${ }^{11}$ For example, in addition to antibodies to hnRNP A1, MS patients develop antibodies to NFs and neurofascin. ${ }^{11,112,200,202}$ In mice, immunization with NF-L caused antibody formation, IgG deposition on axons and spastic paraparesis concurrent with spinal cord axonal degeneration. ${ }^{200,201,239}$ In separate experiments, the addition of anti-neurofascin antibodies following induction of EAE with MOG specific T-cells, augmented disease and showed increased APP expression. ${ }^{112}$ Like antibodies to NF and neurofascin, the data presented here indicate that antibodies to hnRNP A1 contribute to neurodegeneration in EAE and because these antibodies are present in MS patients, strongly support their role in neurodegeneration in MS. ${ }^{11,12,94,95,192,193}$ We believe these data suggest that antihnRNP A1 antibodies contribute to a 'dying back' mechanism of axonal degeneration. Although an exact mechanism in these experiments is not yet apparent, these data suggest that the site of attack of the anti-hnRNP A1 antibodies might be the cells of origins (neuronal cell bodies) of the VSCT, which are present in the lumbosacral spinal cord. In support of this hypothesis are two recent studies in EAE. The first showed that the VSCT is one of several pathways that is preferentially targeted by T-cells and activated microglia early in the course of EAE concurrent with neurodegeneration. ${ }^{148}$ Interestingly, similar findings were present in the corpus callosum ${ }^{148}$, a common lesion site in MS patients. The second showed that entry of pathogenic Th1 and Th17 CD4 ${ }^{+} \mathrm{T}-$ lymphocytes occurs at the L5 lumbar level, and that the damage present in EAE is initiated at this level of the CNS. ${ }^{240,241} \mathrm{We}$ believe that both control and anti-hnRNP A1 antibodies enter the CNS at this level of the spinal cord and that the anti-hnRNP A1 antibodies specifically bind to the VSCT cells of origin (neuronal cell bodies). The antihnRNP A1 antibodies causes changes in function of hnRNP A1 within the neuronal cell body (such as its mislocalization to the cytoplasm), which in turn contributes to distal axonal damage. M9 is hnRNP A1's nucleocytoplasmic transport sequence and binds transportin, which together is part of the non-classical nucleocytoplasmic transport system. ${ }^{12,97,126,192,242-244}$ Disruption of the importin-mediated ('classical') 
nucleocytoplasmic transport system results in neuronal degeneration. ${ }^{245-248}$ Recent data in our lab using an in vitro model of neurodegeneration showed that anti-hnRNP A1 antibodies entered neurons and cause mis-localization of hnRNP A1 to the cytoplasm where it co-localized with stress granules and altered RNA metabolism of spastin. ${ }^{11,206}$ Changes in spastin metabolism cause spasticity ${ }^{12,249}$, a feature of the animals in this study. Future studies are required to evaluate whether anti-hnRNP A1-M9 antibodies utilized the identical mechanism in vivo. 


\section{CHAPTER 6. DISCUSSION}

Multiple Sclerosis (MS) is the most common autoimmune disorder of the central nervous system. MS specifically targets and impairs the central nervous system (CNS) of the individual. The disease course and severity of neurological disability is highly variable between individuals. While the specific cause is unknown, MS is a multifaceted disorder thought to occur in individuals with a predisposed genetic susceptibility in which disease is triggered by an environmental factor such as altered metabolism, a virus, or other environmental factors. Autoantibodies and their ability to target intracellular antigens have become an increasingly important area of research across autoimmune diseases. The predominant theory comprises the idea that initial cellular injury predominates and is immediately followed by intracellular antigens being exposed to the adoptive immune response resulting in the ability of antibody response to the target antigen. Autoantibodies have been implicated in a variety of autoimmune diseases such as SLE, RA, paraneoplastic disorders, MS, and HAM/TSP.

Patients with MS have been shown to produce autoantibodies to the RNA binding protein hnRNP A1. It is important to note that patients with other neurologic diseases and normal controls do not produce autoantibodies to this protein. hnRNP A1 is an RNA binding protein highly expressed in neurons. hnRNP A1 functions in pre-mRNA splicing, RNA metabolism, and nuclear/cytoplasmic trafficking of mature mRNA to sites of translation. hnRNP A1 is a 320 amino acid protein with several functional motifs. The N-terminus of hnRNP A1 is composed of a 185 amino acid residue comprising two 90 amino acid repeats which function as RNA recognition motifs (RRM) or RNA binding domains (RBD). The remaining C-terminus of hnRNP A1 comprises the remaining 135 residues. The C-terminal region is composed of a glycine rich region encoding an $\mathrm{RGG}$ box whose functionality includes RNA binding, protein-protein interactions, nuclear localization, and RNA strand annealing activity. Nearing the end of the C-terminus is the M9 sequence composing amino acids 268-305. The M9 region serves as the nuclear export sequence/nuclear localization sequence (NES/NLS) of the protein. The remaining amino acid residue at the C-terminus of the protein comprises the F peptide (aa 305-320), which must be phosphorylated to initiate the binding of hnRNP A1 to transportin 1 (Trn1), which is necessary for nuclear/cytoplasmic transport. It is important to note that patients with MS produce autoantibodies that present specificity to the M9 region of hnRNP A1.

Although it had been shown that MS patients produce autoantibodies to hnRNP A1, not much had been elucidated regarding mechanism. We sought to reveal the mechanism by which trafficking of anti-hnRNP A1 specific antibodies occurs as well as possible downstream effects due to the specificity of anti-hnRNP A1 antibodies. Previously it has been published that anti-recoverin antibodies, present in patients with cancer associated retinopathy (CAR), penetrate E1A.NR3 cells through endocytosis ${ }^{250}$. We have published data presenting a similar mechanism of entry utilized by anti-hnRNP A1 antibodies into neuronal cells. Through colocalization studies as well as studies utilizing agents to block various forms of endosomal entry, we determined that anti- 
hnRNP A1 antibodies penetrate neuronal cells via clathrin-mediated endocytosis. In comparison to control IgG, anti-hnRNP A1 specific antibodies penetrated neuronal cells more readily and exhibited a higher level of colocalization with early endosomes. Since endocytosis is a general method of cellular uptake of a variety of molecules across various neuronal cell types, antibody penetration could be occurring across a multitude of cell types.

In addition to determining mechanism of antibody entry, we sought to determine any downstream effects that could be occurring due to antibody specificity. We worked to determine if any changes in cellular viability were present due to the addition of antihnRNP A1 specific antibodies compared to control IgG. We determined the viability of the neuronal cells based upon three measures of overall cell health: caspase 3/7 activity as a measure of apoptosis, cytotoxicity of the cell, and ATP levels of the cell. Although there was no differences in cytotoxicity of the cells between groups, we do present data representing a significant increase in caspase $3 / 7$ activity resulting in increased apoptosis and a significant decrease in ATP levels of anti-hnRNP A1 antibody treated cells compared to IgG treated and control cells. Studies have shown that decreases in ATP levels often correlates with initiation of apoptosis leading to cell death ${ }^{172}$. In addition to the altered cellular viability presented, we also present the effect of anti-hnRNP A1 antibodies upon endogenous hnRNP A1 protein. Anti-hnRNP A1 antibodies exhibited a specific effect upon the endogenous protein resulting in redistribution of hnRNP A1 from a predominantly nuclear localization to a near equal nuclear to cytoplasmic redistribution. It is important to note that the epitope recognized by the anti-hnRNP A1 antibodies is specific to amino acids 293-304, the M9 region of hnRNP A1, which serves as the NES/NLS ${ }^{11}$. Studies have shown that deletion mutations within this region of M9 result in loss of nuclear import and export function of hnRNP A1 protein ${ }^{126}$. Taken together, we believe that anti-hnRNP A1 antibodies specificity to the M9 region results in antibody binding to this sequence resulting in similar loss of function as shown by deletion mutations of this region.

In order to further explore the effect of anti-hnRNP A1 antibodies upon cell viability, we examined cellular trafficking of the antibodies after release from early endosomes. Based upon the altered cellular viability of the cells due to antibody presence, we believed that the presence of antibodies would cause formation of stress granules within the neuronal cells. In order to determine trafficking of antibodies within neuronal granules, we needed to perform colocalization studies labeling the three typed of neuronal granules: stress granules (SGs), processing bodies (P bodies), and neuronal transport granules ${ }^{179}$. Stress granule formation occurs within cells due to various cellular stressors and act to harbor various RNA binding proteins and their translationally arrested mRNA cargo ${ }^{179}$. Once the cell has regained a state of homeostasis, SGs sort, remodel, and export their mRNA cargo for reinitiation or storage ${ }^{179}$. P bodies act as sites of translational repression and degradation for the cell ${ }^{179}$. Neuronal granules are transport granules of the neuron and are utilized to transport various mRNA cargo along the axon to various sites of translation ${ }^{179}$. Our studies revealed that anti-hnRNP A1 antibodies as well as control IgG caused stress granule formation within neuronal cells. Additionally, both anti-hnRNP A1 antibodies as well as control IgG colocalized within SGs, however, 
anti-hnRNP A1 antibodies colocalized more readily than control IgG. Furthermore, neither anti-hnRNP A1 antibodies nor control IgG colocalized with either P bodies or neuronal transport granules. Our studies suggest that antibodies contain specificity in binding SGs once they have trafficked into the cytoplasm of neuronal cells.

Based upon our findings, we believed that anti-hnRNP A1 antibodies might have a specific effect upon bound mRNA cargoes of hnRNP A1 protein. During the cellular stress response hnRNP A1 protein enters stress granules, thus protecting its mRNA cargo in a state of translational repression ${ }^{179}$. Since anti-hnRNP A1 antibodies are also present within stress granules we determined specific mRNA binding partners as well as possible downstream effects of anti-hnRNP A1 antibodies upon these mRNAs. Through RNA immunoprecipitation studies utilizing qRT-PCR, we determined that spastin (SPG4) and paraplegin (SPG7) are mRNA binding partners of hnRNP A1 protein. Previous studies have shown that neurons transfected with anti-hnRNP A1 antibodies had altered levels of SPG4 and SPG7 compared to controls ${ }^{12}$. Furthermore, IgG isolated from MS patients presented lowered levels of SPG4 and SPG7 compared to controls when analyzed by RTPCR ${ }^{12}$. Our studies further strengthened the link between anti-hnRNP A1 antibodies and SPG4 and SPG7. We present Western blot data revealing translational repression of SPG4 and SPG7 due to anti-hnRNP A1 antibody addition to neuronal cells. It is important to note that mutations in SPG4 and SPG7 result in various form of HSP, a genetically inherited spastic disorder which is clinically similar to some forms of MS ${ }^{133}$. Mutations in SPG4 account for the most common autosomal dominant form of HSP ${ }^{133}$. SPG7 is associated with mitochondrial function, specifically being implicated in mitochondrial chain complexes I and IV ${ }^{185}$. Patients with HSP resulting from mutations in SPG7 have been shown to have a significant decrease in mitochondrial chain complexes I and IV ${ }^{185}$. Taken together with the previous data presenting altered levels of cellular ATP due to anti-hnRNP A1 addition to neuronal cells, we provide a possible mechanism for ATP depletion. It appears that anti-hnRNP A1 antibodies cause translational repression of various mRNA binding partners of hnRNP A1, specifically SPG7. We hypothesize that the reduction of SPG7 protein levels further causes a depletion of mitochondrial chain complexes I and IV similar to what is seen in HSP patients with mutations in SPG7. Depletion of mitochondrial chain complexes I and IV would result in depletion of ATP levels in the effects cells. We hypothesize that the suggested mechanism of altered cellular viability due to depletion of ATP levels could result in neurodegeneration of effected neuronal cells.

We further explored the question of neurodegeneration in an animal model of MS. We employed the experimental autoimmune encephalomyelitis (EAE) murine model of MS. Disease was initiated in the animals followed by treatment with anti-hnRNP A1-M9 specific antibodies (whose epitope overlap the immunodominant epitope recognized by IgG isolated from MS patients) compared to normal control IgG as well as diseased animals with no antibody addition. Clinical scoring of the animals throughout disease revealed that anti-hnRNP A1 antibody specificity worsens clinical symptoms resulting in a 'spastic' disease state compared to controls. Furthermore, anti-hnRNP A1 antibody treated EAE mice show increased neurodegeneration by Fluoro-jade $\mathrm{C}$ neurodegeneration staining compared to controls. Specifically, anti-hnRNP A1 antibodies caused increased 
neurodegeneration in the genu of the corpus callosum, deep white matter (arbor vitae) of the cerebellum, and the ventral spinocerebellar tract of the brain stem. To sum up our findings in an antibody treated EAE model of MS, anti-hnRNP A1-M9 antibody treatment caused: 1) worsened clinical disease 2) altered clinical phenotype resulting in spastic paraparesis 3) increased neurodegeneration in the distal projection of the VSCT in the deep white matter of the cerebellum and 4) increased levels of neurodegeneration in the genu of the corpus callosum.

We believe that the data presented supports a 'dying-back' mechanism of neurodegeneration due to an antibody-mediated autoimmune response on the CNS. 'Dying back' axonal degeneration is described as a distal to proximal degeneration of the axon due to metabolic, toxic, and neurodegenerative diseases ${ }^{188,217-219}$. Specifically within our model system we describe the most proximal portion of the VSCT, the VSCT cells of origin the spinal cord, as showing no differences in neurodegeneration between anti-hnRNP A1 treated mice compared to controls. The VSCT cells of origin are the most proximal segment of the VSCT contained within laminae VII, VIII, and IX of the lumbosacral spinal cord ${ }^{210-215}$. As we move rostrally, neurodegeneration of the VSCT increased, however there were no differences between the groups in the brainstem. As we continue rostrally, neurodegeneration increases and we witness a significant increase in neurodegeneration of anti-hnRNP A1 antibody treated mice as the VSCT enters the cerebellum and projects into the deep white matter (arbor vitae) of the cerebellum. These segments of the VSCT contain the most distal axons as they synapse on the granular cells of the cerebellum. Importantly, the increased neurodegeneration we show in the distal VSCT projections compared to the proximal segments of the VSCT are consistent with the 'dying back' neurodegeneration phenomena ${ }^{188,217-219}$. Furthermore, the VSCT makes afferent connections to the corticospinal tract via cerebellar efferents. Similar to cerebellar lesions and inherited spastic ataxias causing changes in muscle tone, we suggest that antibody-mediated neurodegeneration of the VSCT might also contribute to neurodegeneration as evidenced by anti-hnRNP A1 antibody treated EAE mice.

In summary, we have presented data implicating anti-hnRNP A1 antibodies in the neurodegeneration seen in an EAE model of MS. We have provided evidence of worsened clinical phenotype resulting in 'spasticity' of anti-hnRNP A1 antibody treated animals. Furthermore, we have correlated worsened phenotype with increased neurodegeneration of the afferent tracts of the VSCT projecting into the deep white matter of the cerebellum in a 'dying back' type phenomena. Our in vitro studies have further provided a mechanism to explain the antibody-induced neurodegeneration. We have mapped the mechanism of antibody entry through clathrin-mediated endocytosis. Data has been presented exhibiting deleterious effects of anti-hnRNP A1 antibodies including redistribution of endogenous hnRNP A1 protein, decreased cellular ATP levels, and increased apoptosis. Additionally, we have evidence suggesting a mechanism by which anti-hnRNP A1 antibodies alter cellular viability leading to neurodegeneration. Our data suggest that anti-hnRNP A1 antibodies enter neuronal cells via clathrinmediated endocytosis. Once inside the cell, antibodies cause cellular stress resulting in formation of SGs to which antibodies localize. Furthermore, anti-hnRNP A1 antibodies presence results in a redistribution of endogenous hnRNP A1 from a primarily nuclear to 
an equal nuclear to cytoplasmic redistribution. The antibody presence has an effect upon bound mRNA binding partners of hnRNP A1 protein, specifically SPG4 and SPG7 mRNA. The antibody effect results in translational repression of SPG4 and SPG7. Similar to mutations in SPG7 resulting in depletion of mitochondrial chain complexes I and IV, we hypothesize that translational repression of SPG7 results in depleted mitochondrial chain complexes I and IV. Depletion of these complexes would result in depleted ATP levels leading to apoptosis and ultimately neurodegeneration as seen in the EAE model. Further work needs to be completed to gain further insight into the intricacies of the antibody-mediated neurodegeneration witnessed throughout our studies, however the work at hand has laid the groundwork for further investigation towards a possible treatment pertaining to the neurodegeneration present in MS. 


\section{LIST OF REFERENCES}

1. Douglas, J. G. L. L. M. Antibodies to an intracellular antigen penetrate neuronal cells and cause deleterious effects. Journal of Clinical and Cellular Immunology (2013).

2. Michael C Levin, J. N. D., Lindsay Meyers, Sangmin Lee, Yoojin Shin, Lidia A Gardner. Neurodegeneration in Multiple sclerosis involves multiple pathogenic mechanisms. Degenerative Neurological and Neuromuscular Disease. 2014:4 4963, doi:http://dx.doi.org/10.2147/DNND.S54391 (2014).

3. Gilgun-Sherki, Y., Melamed, E. \& Offen, D. The role of oxidative stress in the pathogenesis of multiple sclerosis: the need for effective antioxidant therapy. $J$ Neurol 251, 261-268, doi:10.1007/s00415-004-0348-9 (2004).

4. Corthals, A. P. Multiple sclerosis is not a disease of the immune system. $Q$ Rev Biol 86, 287-321 (2011).

5. Lassmann, H. Hypoxia-like tissue injury as a component of multiple sclerosis lesions. J Neurol Sci 206, 187-191, doi:S0022510X02004215 [pii] (2003).

6. Smith, K. J. \& Lassmann, H. The role of nitric oxide in multiple sclerosis. Lancet Neurol 1, 232-241, doi:S1474442202001023 [pii] (2002).

7. Frischer, J. M. et al. The relation between inflammation and neurodegeneration in multiple sclerosis brains. Brain : a journal of neurology 132, 1175-1189, doi:10.1093/brain/awp070 (2009).

8. Bjartmar, C., Wujek, J. R. \& Trapp, B. D. Axonal loss in the pathology of MS: consequences for understanding the progressive phase of the disease. Journal of the neurological sciences 206, 165-171 (2003).

9. Trapp, B. D. \& Nave, K. A. Multiple sclerosis: an immune or neurodegenerative disorder? Anпи Rev Neurosci 31, 247-269 (2008).

10. McFarland, H. F. \& Martin, R. Multiple sclerosis: a complicated picture of autoimmunity. Nat Immunol 8, 913-919, doi:ni1507 [pii] 10.1038/ni1507 (2007).

11. Levin, M. C. et al. Autoantibodies to Non-myelin Antigens as Contributors to the Pathogenesis of Multiple Sclerosis. Journal of clinical \& cellular immunology $\mathbf{4}$, doi:10.4172/2155-9899.1000148 (2013).

12. Lee, S. et al. A potential link between autoimmunity and neurodegeneration in immune-mediated neurological disease. Journal of neuroimmunology 235, 56-69, doi:10.1016/j.jneuroim.2011.02.007 (2011).

13. Lassmann, H. \& van Horssen, J. The molecular basis of neurodegeneration in multiple sclerosis. FEBS Lett 585, 3715-3723, doi:S0014-5793(11)00593-X [pii] 10.1016/j.febslet.2011.08.004 (2011).

14. Kutzelnigg, A. \& Lassmann, H. Cortical lesions and brain atrophy in MS. $J$ Neurol Sci 233, 55-59, doi:S0022-510X(05)00082-1 [pii] 10.1016/j.jns.2005.03.027 (2005).

15. Kornek, B. et al. Multiple sclerosis and chronic autoimmune encephalomyelitis: a comparative quantitative study of axonal injury in active, inactive, and remyelinated lesions. Am J Pathol 157, 267-276, doi:S0002-9440(10)64537-3 [pii] 10.1016/S0002-9440(10)64537-3 (2000). 
16. Geurts, J. J. \& Barkhof, F. Grey matter pathology in multiple sclerosis. Lancet Neurol 7, 841-851, doi:S1474-4422(08)70191-1 [pii] 10.1016/S14744422(08)70191-1 (2008).

17. Dutta, R. et al. Mitochondrial dysfunction as a cause of axonal degeneration in multiple sclerosis patients. Ann Neurol 59, 478-489, doi:10.1002/ana.20736 (2006).

18. Dutta, R. \& Trapp, B. D. Pathogenesis of axonal and neuronal damage in multiple sclerosis. Neurology 68, S22-31; discussion S43-54 (2007).

19. Bennett, J. L. \& Stuve, O. Update on inflammation, neurodegeneration, and immunoregulation in multiple sclerosis: therapeutic implications. Clin Neuropharmacol 32, 121-132, doi:10.1097/WNF.0b013e3181880359 00002826200905000-00001 [pii] (2009).

20. Aboul-Enein, F., Weiser, P., Hoftberger, R., Lassmann, H. \& Bradl, M. Transient axonal injury in the absence of demyelination: a correlate of clinical disease in acute experimental autoimmune encephalomyelitis. Acta Neuropathol 111, 539547, doi:10.1007/s00401-006-0047-y (2006).

21. Silber, E. \& Sharief, M. K. Axonal degeneration in the pathogenesis of multiple sclerosis. J Neurol Sci 170, 11-18, doi:S0022-510X(99)00178-1 [pii] (1999).

22. Trapp, B. D. et al. Axonal transection in the lesions of multiple sclerosis. $N$ Engl J Med 338, 278-285, doi:10.1056/NEJM199801293380502 (1998).

23. Fisniku, L. K. et al. Gray matter atrophy is related to long-term disability in multiple sclerosis. Ann Neurol 64, 247-254, doi:10.1002/ana.21423 (2008).

24. Geurts, J. J. et al. Does high-field MR imaging improve cortical lesion detection in multiple sclerosis? J Neurol 255, 183-191, doi:10.1007/s00415-008-0620-5 (2008).

25. Lassmann, H. \& Lucchinetti, C. F. Cortical demyelination in CNS inflammatory demyelinating diseases. Neurology 70, 332-333, doi:70/5/332 [pii] 10.1212/01.wnl.0000298724.89870.d1 (2008).

26. Lucchinetti, C. F. et al. Clinical and radiographic spectrum of pathologically confirmed tumefactive multiple sclerosis. Brain 131, 1759-1775, doi:awn098 [pii] 10.1093/brain/awn098 (2008).

27. Peterson, J. W. \& Trapp, B. D. Neuropathobiology of multiple sclerosis. Neurol Clin 23, 107-129, vi-vii, doi:S0733-8619(04)00106-9 [pii] 10.1016/j.ncl.2004.09.008 (2005).

28. Mahad, D., Ziabreva, I., Lassmann, H. \& Turnbull, D. Mitochondrial defects in acute multiple sclerosis lesions. Brain 131, 1722-1735, doi:awn105 [pii] 10.1093/brain/awn105 (2008).

29. Confavreaux, C. L., H; McDonald, I; Miller, D; Noseworthy, J; Smith, K; Wekerle, H. McAlpines Multiple Sclerosis. Vol. 4th Edition (2005).

30. Vartanian, T., Li, Y., Zhao, M. \& Stefansson, K. Interferon-gamma-induced oligodendrocyte cell death: implications for the pathogenesis of multiple sclerosis. Mol Med 1, 732-743 (1995).

31. Sotelo, J. On the viral hypothesis of multiple sclerosis: participation of varicellazoster virus. J Neurol Sci 262, 113-116, doi:S0022-510X(07)00465-0 [pii] 10.1016/j.jns.2007.07.001 (2007). 
32. Ebers, G. C. et al. A full genome search in multiple sclerosis. Nat Genet 13, 472476, doi:10.1038/ng0896-472 (1996).

33. Haines, J. L. et al. A complete genomic screen for multiple sclerosis underscores a role for the major histocompatability complex. The Multiple Sclerosis Genetics Group. Nat Genet 13, 469-471, doi:10.1038/ng0896-469 (1996).

34. Black, J. A., Newcombe, J., Trapp, B. D. \& Waxman, S. G. Sodium channel expression within chronic multiple sclerosis plaques. $J$ Neuropathol Exp Neurol 66, 828-837, doi:10.1097/nen.0b013e3181462841 00005072-200709000-00007 [pii] (2007).

35. Craner, M. J. et al. Molecular changes in neurons in multiple sclerosis: altered axonal expression of Nav1.2 and Nav1.6 sodium channels and $\mathrm{Na}+/ \mathrm{Ca} 2+$ exchanger. Proc Natl Acad Sci US A 101, 8168-8173, doi:10.1073/pnas.04027651010402765101 [pii] (2004).

36. Bechtold, D. A. \& Smith, K. J. Sodium-mediated axonal degeneration in inflammatory demyelinating disease. J Neurol Sci 233, 27-35, doi:S0022510X(05)00078-X [pii] 10.1016/j.jns.2005.03.003 (2005).

37. Young, E. A. et al. Imaging correlates of decreased axonal $\mathrm{Na}+/ \mathrm{K}+$ ATPase in chronic multiple sclerosis lesions. Ann Neurol 63, 428-435, doi:10.1002/ana.21381 (2008).

38. Trapp, B. D. \& Stys, P. K. Virtual hypoxia and chronic necrosis of demyelinated axons in multiple sclerosis. Lancet Neurol 8, 280-291, doi:S1474-4422(09)700432 [pii] 10.1016/S1474-4422(09)70043-2 (2009).

39. Ouardouz, M. et al. Depolarization-induced Ca2+ release in ischemic spinal cord white matter involves L-type $\mathrm{Ca} 2+$ channel activation of ryanodine receptors. Neuron 40, 53-63, doi:S0896627303005403 [pii] (2003).

40. Fern, R., Ransom, B. R. \& Waxman, S. G. Voltage-gated calcium channels in CNS white matter: role in anoxic injury. J Neurophysiol 74, 369-377 (1995).

41. Ouardouz, M. et al. Glutamate receptors on myelinated spinal cord axons: II. AMPA and GluR5 receptors. Ann Neurol 65, 160-166, doi:10.1002/ana.21539 (2009).

42. Ouardouz, M. et al. Glutamate receptors on myelinated spinal cord axons: I. GluR6 kainate receptors. Ann Neurol 65, 151-159, doi:10.1002/ana.21533 (2009).

43. Ouardouz, M., Malek, S., Coderre, E. \& Stys, P. K. Complex interplay between glutamate receptors and intracellular $\mathrm{Ca} 2+$ stores during ischaemia in rat spinal cord white matter. J Physiol 577, 191-204, doi:jphysiol.2006.116798 [pii] 10.1113/jphysiol.2006.116798 (2006).

44. Matute, C. et al. The link between excitotoxic oligodendroglial death and demyelinating diseases. Trends Neurosci 24, 224-230, doi:S0166-2236(00)01746$\mathrm{X}$ [pii] (2001).

45. Steinman, L. Multiple sclerosis: a two-stage disease. Nat Immunol 2, 762-764, doi:10.1038/ni0901-762 ni0901-762 [pii] (2001).

46. Ziskin, J. L., Nishiyama, A., Rubio, M., Fukaya, M. \& Bergles, D. E. Vesicular release of glutamate from unmyelinated axons in white matter. Nat Neurosci $\mathbf{1 0}$, 321-330, doi:nn1854 [pii] 10.1038/nn1854 (2007). 
47. Ye, Z. C., Wyeth, M. S., Baltan-Tekkok, S. \& Ransom, B. R. Functional hemichannels in astrocytes: a novel mechanism of glutamate release. $J$ Neurosci 23, 3588-3596, doi:23/9/3588 [pii] (2003).

48. Parpura, V., Scemes, E. \& Spray, D. C. Mechanisms of glutamate release from astrocytes: gap junction "hemichannels", purinergic receptors and exocytotic release. Neurochem Int 45, 259-264, doi:10.1016/j.neuint.2003.12.011 S0197018603002900 [pii] (2004).

49. Taylor, C. T. Interdependent roles for hypoxia inducible factor and nuclear factorkappaB in hypoxic inflammation. J Physiol 586, 4055-4059, doi:jphysiol.2008.157669 [pii] 10.1113/jphysiol.2008.157669 (2008).

50. Schmedtje, J. F., Jr., Ji, Y. S., Liu, W. L., DuBois, R. N. \& Runge, M. S. Hypoxia induces cyclooxygenase-2 via the NF-kappaB p65 transcription factor in human vascular endothelial cells. J Biol Chem 272, 601-608 (1997).

51. Dux, E. et al. The blood-brain barrier in hypoxia: ultrastructural aspects and adenylate cyclase activity of brain capillaries. Neuroscience 12, 951-958, doi:0306-4522(84)90182-9 [pii] (1984).

52. Law, M. et al. Microvascular abnormality in relapsing-remitting multiple sclerosis: perfusion MR imaging findings in normal-appearing white matter. Radiology 231, 645-652, doi:10.1148/radiol.2313030996 231/3/645 [pii] (2004).

53. Ge, Y. et al. Dynamic susceptibility contrast perfusion MR imaging of multiple sclerosis lesions: characterizing hemodynamic impairment and inflammatory activity. AJNR Am J Neuroradiol 26, 1539-1547, doi:26/6/1539 [pii] (2005).

54. Adhya, S. et al. Pattern of hemodynamic impairment in multiple sclerosis: dynamic susceptibility contrast perfusion MR imaging at 3.0 T. Neuroimage 33, 1029-1035, doi:S1053-8119(06)00878-0 [pii] 10.1016/j.neuroimage.2006.08.008 (2006).

55. Inglese, M. et al. Perfusion magnetic resonance imaging correlates of neuropsychological impairment in multiple sclerosis. J Cereb Blood Flow Metab 28, 164-171, doi:9600504 [pii] 10.1038/sj.jcbfm.9600504 (2008).

56. Varga, A. W. et al. White matter hemodynamic abnormalities precede subcortical gray matter changes in multiple sclerosis. J Neurol Sci 282, 28-33, doi:S0022-510X(08)00673-4 [pii] 10.1016/j.jns.2008.12.036 (2009).

57. Graumann, U., Reynolds, R., Steck, A. J. \& Schaeren-Wiemers, N. Molecular changes in normal appearing white matter in multiple sclerosis are characteristic of neuroprotective mechanisms against hypoxic insult. Brain Pathol 13, 554-573 (2003).

58. Greer, S. N., Metcalf, J. L., Wang, Y. \& Ohh, M. The updated biology of hypoxia-inducible factor. $E M B O J 31,2448-2460$, doi:emboj2012125 [pii] 10.1038/emboj.2012.125.

59. Cramer, T. et al. HIF-1 alpha is essential for myeloid cell-mediated inflammation. Cell 112, 645-657, doi:S0092867403001545 [pii] (2003).

60. Karhausen, J. et al. Epithelial hypoxia-inducible factor-1 is protective in murine experimental colitis. J Clin Invest 114, 1098-1106, doi:10.1172/JCI21086 (2004).

61. Belaiba, R. S. et al. Hypoxia up-regulates hypoxia-inducible factor-1alpha transcription by involving phosphatidylinositol 3-kinase and nuclear factor 
kappaB in pulmonary artery smooth muscle cells. Mol Biol Cell 18, 4691-4697, doi:E07-04-0391 [pii] 10.1091/mbc.E07-04-0391 (2007).

62. van Uden, P., Kenneth, N. S. \& Rocha, S. Regulation of hypoxia-inducible factor1alpha by NF-kappaB. Biochem J 412, 477-484, doi:BJ20080476 [pii] 10.1042/BJ20080476 (2008).

63. van Uden, P. et al. Evolutionary conserved regulation of HIF-1 beta by NFkappaB. PLoS Genet 7, e1001285, doi:10.1371/journal.pgen.1001285.

64. Scortegagna, M. et al. HIF-1alpha regulates epithelial inflammation by cell autonomous NFkappaB activation and paracrine stromal remodeling. Blood 111, 3343-3354, doi:blood-2007-10-115758 [pii]10.1182/blood-2007-10-115758 (2008).

65. Dawson, T. M., Sasaki, M., Gonzalez-Zulueta, M. \& Dawson, V. L. Regulation of neuronal nitric oxide synthase and identification of novel nitric oxide signaling pathways. Prog Brain Res 118, 3-11 (1998).

66. Bo, L. et al. Induction of nitric oxide synthase in demyelinating regions of multiple sclerosis brains. Ann Neurol 36, 778-786, doi:10.1002/ana.410360515 (1994).

67. van der Goes, A. et al. Reactive oxygen species are required for the phagocytosis of myelin by macrophages. $J$ Neuroimmunol 92, 67-75, doi:S01655728(98)00175-1 [pii] (1998).

68. Ukkonen, M., Wu, K., Reipert, B., Dastidar, P. \& Elovaara, I. Cell surface adhesion molecules and cytokine profiles in primary progressive multiple sclerosis. Mult Scler 13, 701-707, doi:1352458506075378 [pii] $10.1177 / 1352458506075378$ (2007).

69. Langemann, H., Kabiersch, A. \& Newcombe, J. Measurement of low-molecularweight antioxidants, uric acid, tyrosine and tryptophan in plaques and white matter from patients with multiple sclerosis. Eur Neurol 32, 248-252 (1992).

70. Lu, F. et al. Oxidative damage to mitochondrial DNA and activity of mitochondrial enzymes in chronic active lesions of multiple sclerosis. $J$ Neurol Sci 177, 95-103, doi:S0022-510X(00)00343-9 [pii] (2000).

71. Pacher, P., Beckman, J. S. \& Liaudet, L. Nitric oxide and peroxynitrite in health and disease. Physiol Rev 87, 315-424, doi:87/1/315 [pii] 10.1152/physrev.00029.2006 (2007).

72. Smith, K. J., Kapoor, R., Hall, S. M. \& Davies, M. Electrically active axons degenerate when exposed to nitric oxide. Ann Neurol 49, 470-476 (2001).

73. Parkinson, J. F., Mitrovic, B. \& Merrill, J. E. The role of nitric oxide in multiple sclerosis. J Mol Med (Berl) 75, 174-186 (1997).

74. Callapina, M., Zhou, J., Schmid, T., Kohl, R. \& Brune, B. NO restores HIF1alpha hydroxylation during hypoxia: role of reactive oxygen species. Free Radic Biol Med 39, 925-936, doi:S0891-5849(05)00288-1 [pii] 10.1016/j.freeradbiomed.2005.05.009 (2005).

75. Hagen, T., Taylor, C. T., Lam, F. \& Moncada, S. Redistribution of intracellular oxygen in hypoxia by nitric oxide: effect on HIF1 alpha. Science 302, 1975-1978, doi:10.1126/science.1088805 302/5652/1975 [pii] (2003). 
76. Liu, Q. et al. A Fenton reaction at the endoplasmic reticulum is involved in the redox control of hypoxia-inducible gene expression. Proc Natl Acad Sci U S A 101, 4302-4307, doi:10.1073/pnas.0400265101 0400265101 [pii] (2004).

77. Wang, G. L., Jiang, B. H. \& Semenza, G. L. Effect of altered redox states on expression and DNA-binding activity of hypoxia-inducible factor 1. Biochem Biophys Res Commun 212, 550-556, doi:S0006-291X(85)72005-0 [pii] 10.1006/bbrc.1995.2005 (1995).

78. Schopfer, F. J., Baker, P. R. \& Freeman, B. A. NO-dependent protein nitration: a cell signaling event or an oxidative inflammatory response? Trends Biochem Sci 28, 646-654, doi:S0968-0004(03)00272-X [pii] 10.1016/j.tibs.2003.10.006 (2003).

79. Bizzozero, O. A., DeJesus, G., Bixler, H. A. \& Pastuszyn, A. Evidence of nitrosative damage in the brain white matter of patients with multiple sclerosis. Neurochem Res 30, 139-149 (2005).

80. Dumuis, A., Sebben, M., Haynes, L., Pin, J. P. \& Bockaert, J. NMDA receptors activate the arachidonic acid cascade system in striatal neurons. Nature 336, 6870, doi:10.1038/336068a0 (1988).

81. Gilgun-Sherki, Y., Melamed, E. \& Offen, D. Oxidative stress inducedneurodegenerative diseases: the need for antioxidants that penetrate the blood brain barrier. Neuropharmacology 40, 959-975, doi:S0028390801000193 [pii] (2001).

82. Barkhatova, V. P., Zavalishin, I. A., Askarova, L., Shavratskii, V. \& Demina, E. G. Changes in neurotransmitters in multiple sclerosis. Neurosci Behav Physiol 28, 341-344 (1998).

83. Stover, J. F. et al. Neurotransmitters in cerebrospinal fluid reflect pathological activity. Eur J Clin Invest 27, 1038-1043 (1997).

84. McDonald, J. W., Althomsons, S. P., Hyrc, K. L., Choi, D. W. \& Goldberg, M. P. Oligodendrocytes from forebrain are highly vulnerable to AMPA/kainate receptor-mediated excitotoxicity. Nat Med 4, 291-297 (1998).

85. Matute, C., Sanchez-Gomez, M. V., Martinez-Millan, L. \& Miledi, R. Glutamate receptor-mediated toxicity in optic nerve oligodendrocytes. Proc Natl Acad Sci U $S$ A 94, 8830-8835 (1997).

86. Burnashev, N. Calcium permeability of glutamate-gated channels in the central nervous system. Curr Opin Neurobiol 6, 311-317, doi:S0959-4388(96)80113-9 [pii] (1996).

87. Trowsdale, J. Multiple sclerosis: putting two and two together. Nat Med 12, 11191121, doi:nm1006-1119 [pii] 10.1038/nm1006-1119 (2006).

88. Sospedra, M. \& Martin, R. Molecular mimicry in multiple sclerosis. Autoimmunity 39, 3-8, doi:R07M52Q2Q5305721 [pii] $10.1080 / 08916930500484922$ (2006).

89. Oldstone, M. B. Molecular mimicry and immune-mediated diseases. FASEB J 12, 1255-1265 (1998).

90. Libbey, J. E., McCoy, L. L. \& Fujinami, R. S. Molecular mimicry in multiple sclerosis. Int Rev Neurobiol 79, 127-147, doi:S0074-7742(07)79006-2 [pii] 10.1016/S0074-7742(07)79006-2 (2007). 
91. Hollsberg, P. \& Hafler, D. A. Seminars in medicine of the Beth Israel Hospital, Boston. Pathogenesis of diseases induced by human lymphotropic virus type I infection. N Engl J Med 328, 1173-1182, doi:10.1056/NEJM199304223281608 (1993).

92. Lee, S. \& Levin, M. C. Molecular mimicry in neurological disease: what is the evidence? Cell Mol Life Sci 65, 1161-1175, doi:10.1007/s00018-007-7312-7 (2008).

93. Kalume, F., Lee, S. M., Morcos, Y., Callaway, J. C. \& Levin, M. C. Molecular mimicry: cross-reactive antibodies from patients with immune-mediated neurologic disease inhibit neuronal firing. Journal of neuroscience research 77, 82-89, doi:10.1002/jnr.20137 (2004).

94. Levin, M. C. et al. Autoimmunity due to molecular mimicry as a cause of neurological disease. Nature medicine 8, 509-513, doi:10.1038/nm0502-509 (2002).

95. Douglas, J. N. et al. Antibody transfection into neurons as a tool to study disease pathogenesis. Journal of visualized experiments : JoVE, doi:10.3791/4154 (2012).

96. Lee, S., Shin, Y., Marler, J. \& Levin, M. C. Post-translational glycosylation of target proteins implicate molecular mimicry in the pathogenesis of HTLV-1 associated neurological disease. Journal of neuroimmunology 204, 140-148, doi:10.1016/j.jneuroim.2008.07.020 (2008).

97. Lee, S. M., Dunnavant, F. D., Jang, H., Zunt, J. \& Levin, M. C. Autoantibodies that recognize functional domains of hnRNPA1 implicate molecular mimicry in the pathogenesis of neurological disease. Neuroscience letters 401, 188-193, doi:10.1016/j.neulet.2006.03.016 (2006).

98. Lee, S. M., Morcos, Y., Jang, H., Stuart, J. M. \& Levin, M. C. HTLV-1 induced molecular mimicry in neurological disease. Current topics in microbiology and immunology 296, 125-136 (2005).

99. Levin MC, L. S., Gardner LA, Shin Y, Douglas JN, Groover CJ. Pathogenic mechanisms of neurodegeneration based on the phenotypic expression of progressive forms of immune mediated neurologic disease. Degenerative Neurological and Neuromuscular Disease., 175-187. (2012).

100. Sun, J. B. et al. Autoreactive T and B cells responding to myelin proteolipid protein in multiple sclerosis and controls. European journal of immunology 21, 1461-1468, doi:10.1002/eji.1830210620 (1991).

101. Moller, J. R., Johnson, D., Brady, R. O., Tourtellotte, W. W. \& Quarles, R. H. Antibodies to myelin-associated glycoprotein (MAG) in the cerebrospinal fluid of multiple sclerosis patients. Journal of neuroimmunology 22, 55-61 (1989).

102. Khalil, M. et al. Epitope specificity of serum antibodies directed against the extracellular domain of myelin oligodendrocyte glycoprotein: Influence of relapses and immunomodulatory treatments. Journal of neuroimmunology 174, 147-156, doi:10.1016/j.jneuroim.2006.01.012 (2006).

103. Genain, C. P., Cannella, B., Hauser, S. L. \& Raine, C. S. Identification of autoantibodies associated with myelin damage in multiple sclerosis. Nature medicine 5, 170-175, doi:10.1038/5532 (1999). 
104. Berger, T. et al. Antimyelin antibodies as a predictor of clinically definite multiple sclerosis after a first demyelinating event. The New England journal of medicine 349, 139-145, doi:10.1056/NEJMoa022328 (2003).

105. Lim, E. T. et al. Anti-myelin antibodies do not allow earlier diagnosis of multiple sclerosis. Multiple sclerosis 11, 492-494 (2005).

106. Kuhle, J. et al. Lack of association between antimyelin antibodies and progression to multiple sclerosis. The New England journal of medicine 356, 371-378, doi:10.1056/NEJMoa063602 (2007).

107. Mathey, E., Breithaupt, C., Schubart, A. S. \& Linington, C. Commentary: Sorting the wheat from the chaff: identifying demyelinating components of the myelin oligodendrocyte glycoprotein (MOG)-specific autoantibody repertoire. European journal of immunology 34, 2065-2071, doi:10.1002/eji.200425291 (2004).

108. Bourquin, C. et al. Selective unresponsiveness to conformational B cell epitopes of the myelin oligodendrocyte glycoprotein in $\mathrm{H}-2 \mathrm{~b}$ mice. Journal of immunology 171, 455-461 (2003).

109. Bourquin, C., Iglesias, A., Berger, T., Wekerle, H. \& Linington, C. Myelin oligodendrocyte glycoprotein-DNA vaccination induces antibody-mediated autoaggression in experimental autoimmune encephalomyelitis. European journal of immunology 30, 3663-3671, doi:10.1002/15214141(200012)30:12\&\#60;3663::AID-IMMU3663\&\#62;3.0.CO;2-7 (2000).

110. Ho, P. P. et al. Identification of naturally occurring fatty acids of the myelin sheath that resolve neuroinflammation. Sci Transl Med 4, 137ra173, doi:4/137/137ra73 [pii] 10.1126/scitranslmed.3003831.

111. Vyshkina, T. \& Kalman, B. Autoantibodies and neurodegeneration in multiple sclerosis. Lab Invest 88, 796-807, doi:labinvest200853 [pii] 10.1038/labinvest.2008.53 (2008).

112. Mathey, E. K. et al. Neurofascin as a novel target for autoantibody-mediated axonal injury. The Journal of experimental medicine 204, 2363-2372, doi:10.1084/jem.20071053 (2007).

113. Eikelenboom, M. J. et al. Multiple sclerosis: Neurofilament light chain antibodies are correlated to cerebral atrophy. Neurology 60, 219-223 (2003).

114. Norgren, N. et al. Neurofilament and glial fibrillary acidic protein in multiple sclerosis. Neurology 63, 1586-1590 (2004).

115. Sueoka, E. et al. Autoantibodies against heterogeneous nuclear ribonucleoprotein B1 in CSF of MS patients. Ann Neurol 56, 778-786, doi:10.1002/ana.20276 (2004).

116. Mayeda, A. \& Krainer, A. R. Regulation of alternative pre-mRNA splicing by hnRNP A1 and splicing factor SF2. Cell 68, 365-375, doi:0092-8674(92)90477-T [pii] (1992).

117. Zhang, Q. S., Manche, L., Xu, R. M. \& Krainer, A. R. hnRNP A1 associates with telomere ends and stimulates telomerase activity. $R N A$ 12, 1116-1128, doi:rna.58806 [pii] 10.1261/rna.58806 (2006).

118. Hay, D. C., Kemp, G. D., Dargemont, C. \& Hay, R. T. Interaction between hnRNPA1 and IkappaBalpha is required for maximal activation of NF-kappaBdependent transcription. Mol Cell Biol 21, 3482-3490, doi:10.1128/MCB.21.10.3482-3490.2001 (2001). 
119. Hamilton, B. J., Burns, C. M., Nichols, R. C. \& Rigby, W. F. Modulation of AUUUA response element binding by heterogeneous nuclear ribonucleoprotein A1 in human T lymphocytes. The roles of cytoplasmic location, transcription, and phosphorylation. J Biol Chem 272, 28732-28741 (1997).

120. Berson, A. et al. Cholinergic-associated loss of hnRNP-A/B in Alzheimer's disease impairs cortical splicing and cognitive function in mice. EMBO Mol Med 4, 730-742, doi:10.1002/emmm.201100995.

121. Gao, F. B. \& Taylor, J. P. RNA-binding proteins in neurological disease. Brain Res 1462, 1-2, doi:S0006-8993(12)00936-5 [pii] 10.1016/j.brainres.2012.05.038.

122. Castello, A., Fischer, B., Hentze, M. W. \& Preiss, T. RNA-binding proteins in Mendelian disease. Trends Genet 29, 318-327, doi:S0168-9525(13)00016-4 [pii] 10.1016/j.tig.2013.01.004.

123. McDonald, K. K. et al. TAR DNA-binding protein 43 (TDP-43) regulates stress granule dynamics via differential regulation of G3BP and TIA-1. Hum Mol Genet 20, 1400-1410, doi:ddr021 [pii] 10.1093/hmg/ddr021.

124. Guil, S., Long, J. C. \& Caceres, J. F. hnRNP A1 relocalization to the stress granules reflects a role in the stress response. Mol Cell Biol 26, 5744-5758, doi:26/15/5744 [pii] 10.1128/MCB.00224-06 (2006).

125. Douglas, J. N. G. L., Levin MC. Antibodies to an Intracellular Antigen Penetrate Neuronal Cells and Cause Deleterious Effects. Journal of Clinical and Cellular Immunology (2013).

126. Michael, W. M., Choi, M. \& Dreyfuss, G. A nuclear export signal in hnRNP A1: a signal-mediated, temperature-dependent nuclear protein export pathway. Cell 83, 415-422, doi:0092-8674(95)90119-1 [pii] (1995).

127. Shamoo, Y., Krueger, U., Rice, L. M., Williams, K. R. \& Steitz, T. A. Crystal structure of the two RNA binding domains of human hnRNP A1 at $1.75 \mathrm{~A}$ resolution. Nat Struct Biol 4, 215-222 (1997).

128. Siomi, H. \& Dreyfuss, G. A nuclear localization domain in the hnRNP A1 protein. J Cell Biol 129, 551-560 (1995).

129. Allemand, E. et al. Regulation of heterogenous nuclear ribonucleoprotein A1 transport by phosphorylation in cells stressed by osmotic shock. Proc Natl Acad Sci U S A 102, 3605-3610, doi:0409889102 [pii] 10.1073/pnas.0409889102 (2005).

130. Lukong, K. E., Chang, K. W., Khandjian, E. W. \& Richard, S. RNA-binding proteins in human genetic disease. Trends Genet 24, 416-425, doi:S01689525(08)00173-X [pii] 10.1016/j.tig.2008.05.004 (2008).

131. Colombrita, C. et al. RNA-binding proteins and RNA metabolism: a new scenario in the pathogenesis of Amyotrophic lateral sclerosis. Arch Ital Biol 149, 83-99 (2011).

132. Kim, H. J. et al. Mutations in prion-like domains in hnRNPA2B1 and hnRNPA1 cause multisystem proteinopathy and ALS. Nature 495, 467-473, doi:10.1038/nature11922 (2013).

133. Salinas, S., Proukakis, C., Crosby, A. \& Warner, T. T. Hereditary spastic paraplegia: clinical features and pathogenetic mechanisms. The Lancet. Neurology 7, 1127-1138, doi:10.1016/S1474-4422(08)70258-8 (2008). 
134. Elmore, S. Apoptosis: a review of programmed cell death. Toxicol Pathol 35, 495-516, doi:779478428 [pii] 10.1080/01926230701320337 (2007).

135. Paola Ramoino, A. D., Marco Fato and Cesare Usai. in inTech (2012).

136. Nichols, B. J. \& Lippincott-Schwartz, J. Endocytosis without clathrin coats. Trends Cell Biol 11, 406-412, doi:S0962-8924(01)02107-9 [pii] (2001).

137. Traub, L. M. Tickets to ride: selecting cargo for clathrin-regulated internalization. Nat Rev Mol Cell Biol 10, 583-596, doi:nrm2751 [pii] 10.1038/nrm2751 (2009).

138. Cosker, K. E., Courchesne, S. L. \& Segal, R. A. Action in the axon: generation and transport of signaling endosomes. Curr Opin Neurobiol 18, 270-275, doi:S0959-4388(08)00078-0 [pii] 10.1016/j.conb.2008.08.005 (2008).

139. Fainzilber, M., Budnik, V., Segal, R. A. \& Kreutz, M. R. From synapse to nucleus and back again--communication over distance within neurons. $J$ Neurosci 31, 16045-16048, doi:31/45/16045 [pii] 10.1523/JNEUROSCI.4006-11.2011.

140. Elvira, G. et al. Characterization of an RNA granule from developing brain. $\mathrm{Mol}$ Cell Proteomics 5, 635-651, doi:M500255-MCP200 [pii] 10.1074/mcp.M500255MCP200 (2006).

141. Kato, M. et al. Cell-free formation of RNA granules: low complexity sequence domains form dynamic fibers within hydrogels. Cell 149, 753-767, doi:S00928674(12)00514-4 [pii] 10.1016/j.cell.2012.04.017.

142. Miller, S. D. \& Karpus, W. J. Experimental autoimmune encephalomyelitis in the mouse. Curr Protoc Immunol Chapter 15, Unit 15 11, doi:10.1002/0471142735.im1501s77 (2007).

143. Constantinescu, C. S., Farooqi, N., O'Brien, K. \& Gran, B. Experimental autoimmune encephalomyelitis (EAE) as a model for multiple sclerosis (MS). $\mathrm{Br}$ J Pharmacol 164, 1079-1106, doi:10.1111/j.1476-5381.2011.01302.x.

144. Shiraga, S. \& Adamus, G. Mechanism of CAR syndrome: anti-recoverin antibodies are the inducers of retinal cell apoptotic death via the caspase 9-and caspase 3-dependent pathway. J Neuroimmunol 132, 72-82, doi:S0165572802003144 [pii] (2002).

145. Bradl, M. et al. Neuromyelitis optica: pathogenicity of patient immunoglobulin in vivo. Ann Neurol 66, 630-643, doi:10.1002/ana.21837 (2009).

146. Bennett, J. L. et al. Intrathecal pathogenic anti-aquaporin-4 antibodies in early neuromyelitis optica. Ann Neurol 66, 617-629, doi:10.1002/ana.21802 (2009).

147. Schmued, L. C., Stowers, C. C., Scallet, A. C. \& Xu, L. Fluoro-Jade C results in ultra high resolution and contrast labeling of degenerating neurons. Brain Res 1035, 24-31 (2005).

148. Brown, D. A. \& Sawchenko, P. E. Time course and distribution of inflammatory and neurodegenerative events suggest structural bases for the pathogenesis of experimental autoimmune encephalomyelitis. The Journal of comparative neurology 502, 236-260, doi:10.1002/cne.21307 (2007).

149. Burgess, A. et al. Loss of human Greatwall results in G2 arrest and multiple mitotic defects due to deregulation of the cyclin B-Cdc2/PP2A balance. Proceedings of the National Academy of Sciences of the United States of America 107, 12564-12569, doi:10.1073/pnas.0914191107 (2010).

150. Burkhardt, H. et al. Epitope-specific recognition of type II collagen by rheumatoid arthritis antibodies is shared with recognition by antibodies that are 
arthritogenic in collagen-induced arthritis in the mouse. Arthritis Rheum 46, 23392348, doi:10.1002/art.10472 (2002).

151. Chester, K. A., Lang, B., Gill, J., Vincent, A. \& Newsom-Davis, J. Lambert-Eaton syndrome antibodies: reaction with membranes from a small cell lung cancer xenograft. J Neuroimmunol 18, 97-104, doi:0165-5728(88)90058-6 [pii] (1988).

152. Reeves, W. H., Satoh, M., Wang, J., Chou, C. H. \& Ajmani, A. K. Systemic lupus erythematosus. Antibodies to DNA, DNA-binding proteins, and histones. Rheum Dis Clin North Am 20, 1-28 (1994).

153. Levin, M. C., Lee, S. M., Morcos, Y., Brady, J. \& Stuart, J. Cross-reactivity between immunodominant human T lymphotropic virus type I tax and neurons: implications for molecular mimicry. J Infect Dis 186, 1514-1517, doi:JID020308 [pii] 10.1086/344734 (2002).

154. Aad, G. et al. Search for Higgs boson pair production in the gammagammabb[over ] final state using pp collision data at sqrt[s] $=8 \mathrm{TeV}$ from the ATLAS detector. Phys Rev Lett 114, 081802 (2015).

155. Smeenk, R. J. Antinuclear antibodies: cause of disease or caused by disease? Rheumatology (Oxford) 39, 581-584 (2000).

156. Radic, M. Z. et al. Heterogeneous nuclear ribonucleoprotein P2 is an autoantibody target in mice deficient for Mer, Axl, and Tyro3 receptor tyrosine kinases. J Immunol 176, 68-74, doi:176/1/68 [pii] (2006).

157. Vincent, A. Successful 'passive transfer' of paraneoplastic stiff person syndrome with antibodies to an intracellular antigen. Brain 133, 3164-3165, doi:awq302 [pii] 10.1093/brain/awq302 (2010).

158. Vincent, A. Stiff, twitchy or wobbly: are GAD antibodies pathogenic? Brain 131, 2536-2537, doi:awn221 [pii] 10.1093/brain/awn221 (2008).

159. Magrys, A., Anekonda, T., Ren, G. \& Adamus, G. The role of anti-alpha-enolase autoantibodies in pathogenicity of autoimmune-mediated retinopathy. J Clin Immunol 27, 181-192, doi:10.1007/s10875-006-9065-8 (2007).

160. Geis, C. et al. Stiff person syndrome-associated autoantibodies to amphiphysin mediate reduced GABAergic inhibition. Brain 133, 3166-3180, doi:awq253 [pii] 10.1093/brain/awq253.

161. Douglas, J. N., Gardner, L.A., Lee, S., Shin, Y., Groover, C.J., Levin, M.C. Antibody transfection into neurons as atool to study disease pathogenesis. Journal of Visualized Experiments, doi:10.3791/4154 (2012).

162. Schmid, E. M. \& McMahon, H. T. Integrating molecular and network biology to decode endocytosis. Nature 448, 883-888, doi:10.1038/nature06031 (2007).

163. von Kleist, L. et al. Role of the clathrin terminal domain in regulating coated pit dynamics revealed by small molecule inhibition. Cell 146, 471-484, doi:10.1016/j.cell.2011.06.025 (2011).

164. Declercq, J., Meulemans, S., Plets, E. \& Creemers, J. W. Internalization of proprotein convertase PC7 from plasma membrane is mediated by a novel motif. J Biol Chem 287, 9052-9060, doi:M111.306407 [pii] 10.1074/jbc.M111.306407.

165. Schnitzer, J. E., Oh, P., Pinney, E. \& Allard, J. Filipin-sensitive caveolaemediated transport in endothelium: reduced transcytosis, scavenger endocytosis, and capillary permeability of select macromolecules. J Cell Biol 127, 1217-1232 (1994). 
166. Segal, R. A. Selectivity in neurotrophin signaling: theme and variations. Annu Rev Neurosci 26, 299-330, doi:10.1146/annurev.neuro.26.041002.131421 041002.131421 [pii] (2003).

167. Miller, F. D. \& Kaplan, D. R. On Trk for retrograde signaling. Neuron 32, 767770, doi:S0896-6273(01)00529-3 [pii] (2001).

168. Holzbaur, E. L. Motor neurons rely on motor proteins. Trends Cell Biol 14, 233240, doi:10.1016/j.tcb.2004.03.009 S0962892404000832 [pii] (2004).

169. Ebbing, B. et al. Effect of spastic paraplegia mutations in KIF5A kinesin on transport activity. Hum Mol Genet 17, 1245-1252, doi:ddn014 [pii] 10.1093/hmg/ddn014 (2008).

170. Reid, E. et al. A kinesin heavy chain (KIF5A) mutation in hereditary spastic paraplegia (SPG10). Am J Hum Genet 71, 1189-1194, doi:S0002-9297(07)604121 [pii] 10.1086/344210 (2002).

171. Ferreirinha, F. et al. Axonal degeneration in paraplegin-deficient mice is associated with abnormal mitochondria and impairment of axonal transport. $J$ Clin Invest 113, 231-242, doi:10.1172/JCI20138 (2004).

172. Laird, F. M. et al. Motor neuron disease occurring in a mutant dynactin mouse model is characterized by defects in vesicular trafficking. The Journal of neuroscience : the official journal of the Society for Neuroscience 28, 1997-2005, doi:10.1523/JNEUROSCI.4231-07.2008 (2008).

173. Matsushita, T. et al. Genetic associations with brain cortical thickness in multiple sclerosis. Genes, brain, and behavior 14, 217-227, doi:10.1111/gbb.12190 (2015).

174. Hafler, D. A. Multiple sclerosis. The Journal of clinical investigation 113, 788794, doi:10.1172/JCI21357 (2004).

175. Lee, S. \& Levin, M. Novel somatic single nucleotide variants within the RNA binding protein hnRNP A1 in multiple sclerosis patients. F1000Research 3, 132, doi:10.12688/f1000research.4436.2 (2014).

176. Isobe, N. et al. An ImmunoChip study of multiple sclerosis risk in African Americans. Brain : a journal of neurology 138, 1518-1530, doi:10.1093/brain/awv078 (2015).

177. Ho, P. P. et al. Identification of naturally occurring fatty acids of the myelin sheath that resolve neuroinflammation. Science translational medicine $\mathbf{4}$, 137ra173, doi:10.1126/scitranslmed.3003831 (2012).

178. Srivastava, R. et al. Potassium channel KIR4.1 as an immune target in multiple sclerosis. The New England journal of medicine 367, 115-123, doi:10.1056/NEJMoa1110740 (2012).

179. Kiebler, M. A. \& Bassell, G. J. Neuronal RNA granules: movers and makers. Neuron 51, 685-690, doi:10.1016/j.neuron.2006.08.021 (2006).

180. Burd, C. G. \& Dreyfuss, G. Conserved structures and diversity of functions of RNA-binding proteins. Science 265, 615-621 (1994).

181. JC, L. Identification of in vivo RNA tragets of the RNA-binding proteins Acinus and hnRNP A1. Edinburgh Research Archive (2009).

182. Chomczynski, P. \& Sacchi, N. Single-step method of RNA isolation by acid guanidinium thiocyanate-phenol-chloroform extraction. Analytical biochemistry 162, 156-159, doi:10.1006/abio.1987.9999 (1987). 
183. Gao, F. B. \& Taylor, J. P. RNA metabolism in neurological disease. Brain research 1584, 1-2, doi:10.1016/j.brainres.2014.09.011 (2014).

184. Ramaswami, M., Taylor, J. P. \& Parker, R. Altered ribostasis: RNA-protein granules in degenerative disorders. Cell 154, 727-736, doi:10.1016/j.cell.2013.07.038 (2013).

185. McDermott, C. J. et al. Investigation of mitochondrial function in hereditary spastic paraparesis. Neuroreport 14, 485-488, doi:10.1097/01.wnr.0000058774.36017.cf(2003).

186. Trapp, B. et al. Axonal transection in the lesions of multiple sclerosis. New Eng J Med 338, 278-285 (1998).

187. Lassmann, H., van Horssen, J. \& Mahad, D. Progressive multiple sclerosis: pathology and pathogenesis. Nature reviews. Neurology 8, 647-656, doi:10.1038/nrneurol.2012.168 (2012).

188. Franklin, R. J., ffrench-Constant, C., Edgar, J. M. \& Smith, K. J. Neuroprotection and repair in multiple sclerosis. Nature reviews. Neurology 8, 624-634, doi:10.1038/nrneurol.2012.200 (2012).

189. Bjartmar, C., Kidd, G., Mork, S., Rudick, R. \& Trapp, B. D. Neurological disability correlates with spinal cord axonal loss and reduced $\mathrm{N}$-acetyl aspartate in chronic multiple sclerosis patients. Annals of neurology 48, 893-901 (2000).

190. Lassmann, H., Bruck, W. \& Lucchinetti, C. F. The immunopathology of multiple sclerosis: an overview. Brain Pathol 17, 210-218 (2007).

191. Ferguson, B., Matyszak, M. K., Esiri, M. M. \& Perry, V. H. Axonal damage in acute multiple sclerosis lesions. Brain 120 ( Pt 3), 393-399 (1997).

192. Levin, M. C. et al. Pathogenic mechanisms of neurodegeneration based on the phenotypic expression of progressive forms of immune-mediated neurologic disease. Degenerative Neurological and Neuromuscular Disease 2, 175-187 (2012).

193. Levin, M. C. et al. Neurodegeneration in multiple sclerosis involves multiple pathogenic mechanisms. Degenerative Neurological and Neuromuscular Disease 4, 49-63, doi:http://dx.doi.org/10.2147/DNND.S5439 (2014).

194. Soulika, A. M. et al. Initiation and progression of axonopathy in experimental autoimmune encephalomyelitis. J Neurosci 29, 14965-14979, doi:10.1523/JNEUROSCI.3794-09.2009 (2009).

195. Nikic, I. et al. A reversible form of axon damage in experimental autoimmune encephalomyelitis and multiple sclerosis. Nat Med 17, 495-499, doi:10.1038/nm.2324 (2011).

196. Rawes, J. A., Calabrese, V. P., Khan, O. A. \& DeVries, G. H. Antibodies to the axolemma-enriched fraction in the cerebrospinal fluid and serum of patients with multiple sclerosis and other neurological diseases. Mult Scler 3, 363-369 (1997).

197. Norgren, N., Edelstam, A. \& Stigbrand, T. Cerebrospinal fluid levels of neurofilament light in chronic experimental autoimmune encephalomyelitis. Brain Res Bull 67, 264-268 (2005).

198. Sadatipour, B. T., Greer, J. M. \& Pender, M. P. Increased circulating antiganglioside antibodies in primary and secondary progressive multiple sclerosis. Ann Neurol 44, 980-983 (1998). 
199. Owens, G. P. et al. Antibodies produced by clonally expanded plasma cells in multiple sclerosis cerebrospinal fluid. Ann Neurol 65, 639-649, doi:10.1002/ana.21641 (2009).

200. Huizinga, R. et al. Immunization with neurofilament light protein induces spastic paresis and axonal degeneration in Biozzi ABH mice. J Neuropathol Exp Neurol 66, 295-304, doi:10.1097/nen.0b013e318040ad5c (2007).

201. Huizinga, R., Gerritsen, W., Heijmans, N. \& Amor, S. Axonal loss and gray matter pathology as a direct result of autoimmunity to neurofilaments. Neurobiol Dis 32, 461-470, doi:10.1016/j.nbd.2008.08.009 (2008).

202. Derfuss, T., Linington, C., Hohlfeld, R. \& Meinl, E. Axo-glial antigens as targets in multiple sclerosis: implications for axonal and grey matter injury. $J \mathrm{Mol} \mathrm{Med}$ (Berl) 88, 753-761, doi:10.1007/s00109-010-0632-3 (2010).

203. Alami, N. H. et al. Axonal Transport of TDP-43 mRNA Granules Is Impaired by ALS-Causing Mutations. Neuron 81, 536-543, doi:10.1016/j.neuron.2013.12.018 (2014).

204. Dreyfuss, G., Kim, V. N. \& Kataoka, N. Messenger-RNA-binding proteins and the messages they carry. Nat Rev Mol Cell Biol 3, 195-205 (2002).

205. Lee, B. J. et al. Rules for nuclear localization sequence recognition by karyopherin beta 2. Cell 126, 543-558 (2006).

206. Douglas, J., Gardner, L. \& Levin, M. C. Antibodies to an intracellular antigen penetrate neuronal cells and cause deleterious effects. J Clinical \& Cellular Immunology 4, 134, doi:10.4172/2155-9899.1000134 (2013).

207. Gu, J., Congdon, E. E. \& Sigurdsson, E. M. Two novel Tau antibodies targeting the 396/404 region are primarily taken up by neurons and reduce Tau protein pathology. J Biol Chem 288, 33081-33095, doi:10.1074/jbc.M113.494922 (2013).

208. Mohamed, H. A. et al. Immunoglobulin Fc gamma receptor promotes immunoglobulin uptake, immunoglobulin-mediated calcium increase, and neurotransmitter release in motor neurons. J Neurosci Res 69, 110-116, doi:10.1002/jnr.10271 (2002).

209. Dubois, B. et al. Resistance of young gelatinase B-deficient mice to experimental autoimmune encephalomyelitis and necrotizing tail lesions. The Journal of clinical investigation 104, 1507-1515, doi:10.1172/JCI6886 (1999).

210. Snyder, R. L., Faull, R. L. \& Mehler, W. R. A comparative study of the neurons of origin of the spinocerebellar afferents in the rat, cat and squirrel monkey based on the retrograde transport of horseradish peroxidase. The Journal of comparative neurology 181, 833-852, doi:10.1002/cne.901810409 (1978).

211. Matsushita, M. \& Hosoya, Y. Cells of origin of the spinocerebellar tract in the rat, studied with the method of retrograde transport of horseradish peroxidase. Brain research 173, 185-200 (1979).

212. Matsushita, M., Hosoya, Y. \& Ikeda, M. Anatomical organization of the spinocerebellar system in the cat, as studied by retrograde transport of horseradish peroxidase. J Comp Neurol 184, 81-106, doi:10.1002/cne.901840106 (1979).

213. Berretta, S., Perciavalle, V. \& Poppele, R. E. Origin of spinal projections to the anterior and posterior lobes of the rat cerebellum. The Journal of comparative neurology 305, 273-281, doi:10.1002/cne.903050208 (1991). 
214. Watson, C. \& Harrison, M. The location of the major ascending and descending spinal cord tracts in all spinal cord segments in the mouse: actual and extrapolated. Anatomical record 295, 1692-1697, doi:10.1002/ar.22549 (2012).

215. Stecina, K., Fedirchuk, B. \& Hultborn, H. Information to cerebellum on spinal motor networks mediated by the dorsal spinocerebellar tract. The Journal of physiology 591, 5433-5443, doi:10.1113/jphysiol.2012.249110 (2013).

216. Shrestha, S. S. et al. Excitatory inputs to four types of spinocerebellar tract neurons in the cat and the rat thoraco-lumbar spinal cord. The Journal of physiology 590, 1737-1755, doi:10.1113/jphysiol.2011.226852 (2012).

217. Murray, L. M., Thomson, D., Conklin, A., Wishart, T. M. \& Gillingwater, T. H. Loss of translation elongation factor (eEF1A2) expression in vivo differentiates between Wallerian degeneration and dying-back neuronal pathology. Journal of anatomy 213, 633-645, doi:10.1111/j.1469-7580.2008.01007.x (2008).

218. Benarroch, E. E. Acquired axonal degeneration and regeneration: Recent insights and clinical correlations. Neurology 84, 2076-2085, doi:10.1212/WNL.0000000000001601 (2015).

219. Coleman, M. Axon degeneration mechanisms: commonality amid diversity. Nature reviews. Neuroscience 6, 889-898, doi:10.1038/nrn1788 (2005).

220. Votsi, C., Zamba-Papanicolaou, E., Middleton, L. T., Pantzaris, M. \& Christodoulou, K. A novel GBA2 gene missense mutation in spastic ataxia. Ann Hum Genet 78, 13-22, doi:10.1111/ahg.12045 (2014).

221. Dogonowski, A. M. et al. Multiple sclerosis impairs regional functional connectivity in the cerebellum. NeuroImage. Clinical 4, 130-138, doi:10.1016/j.nicl.2013.11.005 (2014).

222. Khundadze, M. et al. A hereditary spastic paraplegia mouse model supports a role of ZFYVE26/SPASTIZIN for the endolysosomal system. PLoS Genet $\mathbf{9}$, e1003988, doi:10.1371/journal.pgen.1003988 (2013).

223. MacKenzie-Graham, A. et al. Cortical atrophy in experimental autoimmune encephalomyelitis: in vivo imaging. Neuroimage 60, 95-104, doi:10.1016/j.neuroimage.2011.11.099 (2012).

224. Tanabe, S. \& Yamashita, T. Repulsive guidance molecule-a is involved in Th17cell-induced neurodegeneration in autoimmune encephalomyelitis. Cell reports $\mathbf{9}$, 1459-1470, doi:10.1016/j.celrep.2014.10.038 (2014).

225. Sorbara, C. D. et al. Pervasive axonal transport deficits in multiple sclerosis models. Neuron 84, 1183-1190, doi:10.1016/j.neuron.2014.11.006 (2014).

226. Ganter, P., Prince, C. \& Esiri, M. M. Spinal cord axonal loss in multiple sclerosis: a post-mortem study. Neuropathol Appl Neurobiol 25, 459-467 (1999).

227. Lovas, G., Szilagyi, N., Majtenyi, K., Palkovits, M. \& Komoly, S. Axonal changes in chronic demyelinated cervical spinal cord plaques. Brain 123 ( Pt 2), 308-317 (2000).

228. DeLuca, G. C., Ebers, G. C. \& Esiri, M. M. Axonal loss in multiple sclerosis: a pathological survey of the corticospinal and sensory tracts. Brain 127, 1009-1018 (2004).

229. DeLuca, G. C., Williams, K., Evangelou, N., Ebers, G. C. \& Esiri, M. M. The contribution of demyelination to axonal loss in multiple sclerosis. Brain 129, 1507-1516 (2006). 
230. Evangelou, N., DeLuca, G. C., Owens, T. \& Esiri, M. M. Pathological study of spinal cord atrophy in multiple sclerosis suggests limited role of local lesions.

Brain 128, 29-34, doi:awh323 [pii] 10.1093/brain/awh323 (2005).

231. Weier, K. et al. The role of the cerebellum in multiple sclerosis. Cerebellum 14, 364-374, doi:10.1007/s12311-014-0634-8 (2015).

232. Magliozzi, R. et al. A Gradient of neuronal loss and meningeal inflammation in multiple sclerosis. Ann Neurol 68, 477-493, doi:10.1002/ana.22230 (2010).

233. Dziedzic, T. et al. Wallerian degeneration: a major component of early axonal pathology in multiple sclerosis. Brain Pathol 20, 976-985, doi:10.1111/j.17503639.2010.00401.x (2010).

234. Friese, M. A., Schattling, B. \& Fugger, L. Mechanisms of neurodegeneration and axonal dysfunction in multiple sclerosis. Nature reviews. Neurology 10, 225-238, doi:10.1038/nrneurol.2014.37 (2014).

235. Campbell, G. R. et al. Mitochondrial DNA deletions and neurodegeneration in multiple sclerosis. Ann Neurol 69, 481-492, doi:10.1002/ana.22109 (2011).

236. Haider, L. et al. Oxidative damage in multiple sclerosis lesions. Brain 134, 19141924, doi:awr128 [pii] 10.1093/brain/awr128 (2011).

237. Dutta, R. \& Trapp, B. Mechanisms of neuronal dysfunction and degeneration in multiple sclerosis. Progress in Neurobiology 93, 1-12 (2011).

238. Waxman, S. G. Axonal conduction and injury in multiple sclerosis: the role of sodium channels. Nat Rev Neurosci 7, 932-941 (2006).

239. Huizinga, R., Linington, C. \& Amor, S. Resistance is futile: antineuronal autoimmunity in multiple sclerosis. Trends Immunol 29, 54-60, doi:10.1016/j.it.2007.11.002 (2008).

240. Kamimura, D. et al. The gateway theory: bridging neural and immune interactions in the CNS. Frontiers in neuroscience 7, 204, doi:10.3389/fnins.2013.00204 (2013).

241. Arima, Y. et al. Regional neural activation defines a gateway for autoreactive $\mathrm{T}$ cells to cross the blood-brain barrier. Cell 148, 447-457, doi:10.1016/j.cell.2012.01.022 (2012).

242. Cook, A., Bono, F., Jinek, M. \& Conti, E. Structural biology of nucleocytoplasmic transport. Annu Rev Biochem 76, 647-671 (2007).

243. Cammas, A. et al. Cytoplasmic relocalization of heterogeneous nuclear ribonucleoprotein A1 controls translation initiation of specific mRNAs. Mol Biol Cell 18, 5048-5059, doi:10.1091/mbc.E07-06-0603 (2007).

244. Lee, S. \& Levin, M. Novel somatic single nucleotide variants within the RNA binding protein hnRNP A1 in multiple sclerosis patients [v2; ref status: indexed]. Vol. 3 (2014).

245. Hanz, S. et al. Axoplasmic importins enable retrograde injury signaling in lesioned nerve. Neuron 40, 1095-1104 (2003).

246. Thompson, K. R. et al. Synapse to nucleus signaling during long-term synaptic plasticity; a role for the classical active nuclear import pathway. Neuron 44, 997 1009 (2004).

247. Yudin, D. et al. Localized regulation of axonal RanGTPase controls retrograde injury signaling in peripheral nerve. Neuron 59, 241-252 (2008). 
248. Kim, J. Y. et al. HDAC1 nuclear export induced by pathological conditions is essential for the onset of axonal damage. Nat Neurosci 13, 180-189, doi:nn.2471 [pii] 10.1038/nn.2471 (2010).

249. Roll-Mecak, A. \& Vale, R. D. Structural basis of microtubule severing by the hereditary spastic paraplegia protein spastin. Nature 451, 363-367 (2008).

250. Adamus, G., Amundson, D., Seigel, G. M. \& Machnicki, M. Anti-enolase-alpha autoantibodies in cancer-associated retinopathy: epitope mapping and cytotoxicity on retinal cells. Journal of autoimmunity 11, 671-677, doi:10.1006/jaut.1998.0239 (1998).

251. Hooke Laboratories. Appendix A:Mouse EAE Scoring Guide.Hooke Laboratories. 2015. Version 2015. 


\section{VITA}

Joshua Nathan Douglas was born in 1987. He grew up in Collierville, TN, and graduated from Collierville High School in 2005. He attended the University of Memphis for his undergraduate studies, where he graduated in 2009 with a Bachelor of Science degree in Biology. After that, he continued his passion for science by enrolling in the University of Tennessee Health Science Center in the fall of 2010 to pursue a graduate degree in Biology. Working in the lab of Michael Levin M.D., he completed research tailored to the study of antibodies to the RNA binding protein hnRNP A1. His studies involved various immunologic techniques involving protein, molecular, and microscopy assays. Throughout his tenure in the Levin lab, he published multiple papers including one review, one techniques paper, and multiple research articles based upon work he directly performed in the lab. Joshua will graduate in May 2016 with a Ph.D. in Neuroscience from the University of Tennessee Health Science Center. He will continue his career in science as a Technical Support Representative with one of the leading scientific corporations in the world. 